\title{
Construction of projective special Kähler manifolds
}

\author{
Mauro Mantegazza' ${ }^{1}$
}

Received: 2 February 2021 / Accepted: 27 February 2021 / Published online: 7 April 2021

(c) The Author(s) 2021

\begin{abstract}
In this paper, we present an intrinsic characterisation of projective special Kähler manifolds in terms of a symmetric tensor satisfying certain differential and algebraic conditions. We show that this tensor vanishes precisely when the structure is locally isomorphic to a standard projective special Kähler structure on $\mathrm{SU}(n, 1) / \mathrm{S}(\mathrm{U}(n) \mathrm{U}(1))$. We use this characterisation to classify 4-dimensional projective special Kähler Lie groups.
\end{abstract}

Mathematics Subject Classification 53C55 $\cdot 53 \mathrm{C} 26 \cdot 22 \mathrm{E} 25 \cdot 53 \mathrm{C} 80$

\section{Introduction}

Projective special Kähler manifolds are a special class of Kähler quotients of conic special Kähler manifolds which is a class of pseudo-Kähler manifolds endowed with a symplectic, flat, torsion-free connection and an infinitesimal homothety.

Explicit examples can be found in [1], where homogeneous projective special Kähler manifolds of semisimple Lie groups are classified. A notable case appearing in this list is the complex hyperbolic $n$-space. Many projective special Kähler manifolds can be constructed via the so called r-map [15], which is a construction arising from supergravity and string theory allowing to build a projective special Kähler manifold starting from a homogeneous cubic polynomial. See [13] for a classification of 6-dimensional manifolds that can be constructed via the r-map. Another example is obtained by taking the Weil-Petersson metric on the space of complex structure deformations on a Calabi-Yau 3-dimensional manifold [12].

Projective special Kähler manifolds appear in the study of supergravity and mirror symmetry with the name local special Kähler manifolds (see [19] and [20] for more details on their history and applications to physics, and in particular [8] for their importance in mirror symmetry). The name projective special Kähler was given by Freed in [20] where he also shows how such manifolds are quotients of special Kähler ones [20, Proposition 4.6, p. 20] (see, e.g. [2] for the relation between this definition and the one we will use in this work).

Mauro Mantegazza

mauro.mantegazza.uni@gmail.com

1 Dipartimento di matematica e applicazioni, Università degli studi Milano-Bicocca, via Roberto Cozzi, 55, Bldg.U5, 20125 Milano, Italy 
Projective special Kähler manifolds are not only interesting on their own, as they find an important application in quaternion Kähler geometry. The construction known as c-map, also arising from the same areas of physics, allows in fact to create quaternion Kähler manifolds of negative scalar curvature starting from projective special Kähler ones [15, 3, 4, 26, 9, 18, 23]. Quaternion Kähler manifolds are orientable smooth Riemannian manifolds of dimension $4 n$ with $n \geq 2$, whose holonomy group is a subgroup of $\operatorname{Sp}(n) \operatorname{Sp}(1)$ not contained in $\operatorname{Sp}(n)$. They are important since they are a special family of Einstein manifolds with non-vanishing Ricci tensor, corresponding to one of the possible holonomy groups of a locally irreducible, non-locally symmetric, simply connected Riemannian manifold in Berger's list (see [7]).

In this paper, we present a characterisation of projective special Kähler manifolds that will hopefully shed more light on this type of structure. Our characterisation is intrinsic in the sense that we reduce the projective special Kähler structure to data solely defined on the manifold itself. The characterisation is obtained by means of a locally defined symmetric tensor that we call deviance, satisfying certain conditions: a differential one and an algebraic one. The deviance tensor emerges from the difference between two naturally occurring connections on the conic special Kähler manifold over a projective special Kähler one. Our description arises by writing the two connections with respect to a local frame, and relating the difference to a tensor defined on the basis. We also prove a lower bound for the scalar curvature, which is reached exactly when the deviance is zero; this condition characterises projective special Kähler manifolds isomorphic to the complex hyperbolic $n$-space if one assumes the manifold complete, connected and simply connected. For projective special Kähler manifolds of elliptic type, i.e. such that the corresponding conic special Kähler metric is positive definite, we have a similar result in [6, Theorem 16, p. 126], where complex projective spaces are characterised as the only complete projective special Kähler domains of elliptic type. In that context, in fact, the vanishing of the deviance is induced by the completeness condition.

Our characterisation provides a simpler way to construct projective special Kähler manifolds, and we display this by classifying all possible projective special Kähler structures on 4-dimensional Lie groups. Our classification relies on the classification of Kähler Lie groups by Ovando [28].

We note that an intrinsic characterisation of projective special Kähler Lie groups has been obtained independently in a very recent paper by Macia and Swann [25]. Our setting is slightly more general, since in our study of projective special Kähler Lie groups we do not assume the deviance tensor to be left-invariant. In [25] it is also shown that projective special Kähler Lie groups determine quaternion Kähler Lie groups via the c-map, if one assumes the exactness of the Kähler form and the invariance of the flat connection. A similar result, holding in the case that the projective special Kähler Lie group is the quotient of an affine special Kähler domain, can be deduced from the more general result [16, Corollary 24, p. 33].

Since we are ultimately interested in the c-map, throughout this paper we adopt the same convention as [15], where we only consider projective special Kähler manifolds obtained from conic special Kähler manifolds with signature $(2 n, 2)$. Nonetheless, our characterisation can be generalised to generic signatures. It is worth mentioning that the deviance, being a symmetric tensor of type $(3,0)$, can often be seen as a homogeneous polynomial of degree three, which may have a role in providing a partial inversion to the r-map.

We also use our characterisation to show that, on a Kähler manifold, the existence of a symmetric tensor satisfying the deviance conditions implies the existence of a whole 
family of projective special Kähler structures and we provide sufficient conditions for said structures to be isomorphic.

\section{Definitions}

In this section, we are introducing the basic objects that we are going to discuss in this work.

The coming definition involves a flat connection $\nabla$ and its exterior covariant derivative operator $d^{\nabla}$.

Definition 2.1 A conic special Kähler manifold is the data of a pseudo-Kähler manifold $(\widetilde{M}, \widetilde{g}, \widetilde{I}, \widetilde{\omega})$ with a flat, torsion-free, symplectic connection $\nabla$ and a vector field $\xi$ such that

1. $d^{\nabla} \widetilde{I}=0$ where we interpret $\widetilde{I}$ as a 1 -form with values in $T \widetilde{M}$;

2. $\widetilde{g}(\xi, \xi)$ is nowhere vanishing;

3. $\nabla \xi=\widetilde{\nabla}^{L C} \xi=\mathrm{id}$

4. $\tilde{g}$ is negative definite on $\langle\xi, I \xi\rangle$ and positive definite on its orthogonal complement.

Here $\widetilde{\nabla}^{L C}$ is the Levi-Civita connection.

We will adopt the convention $\widetilde{\omega}=\widetilde{g}(\widetilde{I} \cdot, \cdot)$. Definition 2.1 is identical to Definition 3 in [15] if we take $-g$ as metric.

We start by showing how the Lie derivative along $\xi$ and $I \xi$ in a conic special Kähler manifold behaves on the Kähler structure.

Lemma 2.2 (Lemma 3.2, p. 1336 in [26]) Let $(\widetilde{M}, \widetilde{g}, \widetilde{I}, \widetilde{\omega}, \nabla, \xi)$ be a conic special Kähler manifold, then:

1. $\quad \xi$ is a homothety of scaling factor 2 preserving $\widetilde{I}$;

2. $\widetilde{I} \xi$ preserves the Kähler structure.

Proof See, e.g. [26] where $X=-I \xi$.

Before proceeding, we write the following lemma for future reference.

Lemma 2.3 In a conic special Kähler manifold $(\widetilde{M}, \widetilde{g}, \widetilde{I}, \widetilde{\omega}, \nabla, \xi), \nabla(\widetilde{I} \xi)=\widetilde{I}$.

Proof For all $X \in \mathfrak{X}(\tilde{M})$,

$$
\begin{aligned}
\nabla_{X}(\widetilde{I} \xi)-\widetilde{I} X & =\left(\nabla_{X} \widetilde{I}\right) \xi+\widetilde{I} \nabla_{X} \xi-\widetilde{I} X=\left(\nabla_{X} \widetilde{I}\right) \xi=\left(\nabla_{\xi} \widetilde{I}\right) X \\
& \left.=\nabla_{\xi} \widetilde{I} X\right)-\widetilde{I} \nabla_{\xi} X=\nabla_{\widetilde{I} X}(\xi)+[\xi, \widetilde{I} X]-\widetilde{I}\left(\nabla_{X} \xi+[\xi, X]\right) \\
& =\widetilde{I} X+\mathcal{L}_{\xi}(\widetilde{I} X)-\widetilde{I} X-\widetilde{I} \mathcal{L}_{\xi} X=\left(\mathcal{L}_{\xi} \widetilde{I}\right) X=0 .
\end{aligned}
$$


If we compare Definition 2.1 with Definition 3.1 in [26], we notice that the main difference is the signature of the metric: it is enough to add condition 4 to the latter and to define $X=-I \xi$ in order to obtain two equivalent definitions. The proof of the equivalence is obtained by Lemma 2.3.

Definition 2.4 A projective special Kähler manifold is a Kähler manifold $M$ endowed with a $\mathbb{C}^{*}$-bundle $\pi: \widetilde{M} \rightarrow M$ with $(\widetilde{M}, \widetilde{g}, \widetilde{I}, \widetilde{\omega}, \nabla, \xi)$ conic special Kähler such that $\xi$ and $I \xi$ are the fundamental vector fields associated to $1, i \in \mathbb{C}$, respectively, and $M$ is the Kähler quotient with respect to the induced $\mathrm{U}(1)$-action. In this case, we say that $M$ has a projective special Kähler structure.

For brevity, we will often denote a projective special Kähler manifold by $(\pi: \widetilde{M} \rightarrow M, \nabla)$.

Remark 2.5 We shall see later that by construction, the action is always Hamiltonian with moment map $-\widetilde{g}(\xi, \xi)$, and the choice of the level set affects the quotient only up to scaling.

Concerning the notation for projective special Kähler manifolds as in Definition 2.4, when a tensor or a connection is possessed by both $\widetilde{M}$ and $M$, we will write them and everything concerning them (torsion, curvature forms, covariant exterior differentials) on $\widetilde{M}$ with $\widetilde{(\cdot)}$ above, whereas the corresponding objects on $M$ will be denoted without it.

\section{Difference tensor}

This section is devoted to the tensor obtained as difference between the flat and Levi-Civita connection on a conic special Kähler manifold. We present the known symmetry of this tensor and write the flatness condition in terms of it [20, p. 9-11].

Before talking about the difference tensor, we will introduce some notation and definitions. Following [29], if $V$ is a complex representation with a real structure $\sigma$, we define

$$
[V]:=\{v \in V \mid \sigma(v)=v\} .
$$

Otherwise, for any complex representation $V$,

$$
\llbracket V \rrbracket:=[V \oplus \bar{V}]
$$

where $\bar{V}$ is the conjugate representation of $V$. In particular, the following complex Lie algebra isomorphisms hold:

$$
[V] \otimes_{\mathbb{R}} \mathbb{C} \cong V, \quad \llbracket V \rrbracket \otimes_{\mathbb{R}} \mathbb{C} \cong V \oplus \bar{V}
$$

The same notation is used for the associated vector bundles.

Given an almost complex manifold $(M, I)$, let $T_{1,0} M$ be the holomorphic cotangent bundle. For all $p \in \mathbb{N}$, we denote its $p$-th symmetric power by $S_{p, 0} M$.

Given a (pseudo-)Riemannian manifold $(M, g)$, we denote by $b$ and $\sharp$ the musical isomorphisms induced by $g$, and we can define the following isomorphism

$$
b_{2}=\mathrm{id} \otimes b \otimes \mathrm{id}: T^{*} M \otimes T M \otimes T^{*} M \rightarrow T_{3} M
$$

with inverse $\sharp_{2}:=\mathrm{id} \otimes \sharp \otimes$ id. 
Returning now to the main topic of this section, let $(\tilde{M}, \widetilde{g}, \widetilde{I}, \widetilde{\omega}, \nabla, \xi)$ be a conic special Kähler manifold of dimension $n+1$. We define $\tilde{\eta}$ as the (1,2)-tensor such that for all vector fields $X, Y$ on $\widetilde{M}$ we have $\tilde{\eta}_{X} Y=\nabla_{X} Y-\widetilde{\nabla}_{X}^{L C} Y$, where the employed notation $\widetilde{\eta}_{X} Y$ means $\widetilde{\eta}(X, Y)$.

Consider frames adapted to the pseudo-Kähler structure, hence such that the linear model is $\left(\mathbb{R}^{2 n+2}, g_{0}, I_{0}, \omega_{0}\right)$, where $g_{0}=\sum_{k=1}^{2 k}\left(e^{k}\right)^{2}-\left(e^{2 n+1}\right)^{2}-\left(e^{2 n+2}\right)^{2}, I_{0} e_{2 k-1}=e_{2 k}$ for $k=1, \ldots, n+1$ and $\omega_{0}=g_{0}\left(I_{0} \cdot, \cdot\right)$. Let $\omega^{\nabla}$ and $\widetilde{\omega}^{L C}$ be the connection forms corresponding, respectively, to the flat and the Levi-Civita connections represented with respect to an adapted frame. Thus, we have

$$
\omega^{\nabla}=\widetilde{\omega}^{L C}+\tilde{\eta}
$$

We know that by the theory of Hessian manifolds, $\tilde{\eta}$ is symmetric (see, e.g. [30, §3, p. 194] or $[31, \S 1$, p. 736]). More precisely, we recall the following result (see [20, Proposition 1.34, p. 39], or [5, Proposition 4, p. 1743] and [5, Lemma 3, p. 1745]).

Lemma 3.1 On a conic special Kähler manifold $(\widetilde{M}, \widetilde{g}, \widetilde{I}, \widetilde{\omega}, \nabla, \xi)$, the tensor $\tilde{\eta}$ is a section of $\sharp_{2} \llbracket S_{3,0} \tilde{M} \rrbracket$.

In proving this lemma, one finds the following equality (see, e.g. [5, (3.3), p. 1743]), which we write for further reference.

$$
\nabla \widetilde{I}=[\tilde{\eta}, \tilde{I}]=-2 \widetilde{I} \widetilde{\eta}
$$

Using the flatness of $\nabla$, we observe:

$$
0=\Omega^{\nabla}=\widetilde{\Omega}^{L C}+\widetilde{d}^{L C} \tilde{\eta}+\frac{1}{2}[\tilde{\eta} \wedge \tilde{\eta}],
$$

where $\widetilde{\Omega}^{L C}$ and $\widetilde{d}^{L C}$ are, respectively, the curvature and exterior covariant derivative of the Levi-Civita connection on $\widetilde{M}$.

Arguing as in [20, Proposition 1.34 (a), p. 39] (see also [5, Proposition 4 (iii), p. 1743]), one obtains

Proposition 3.2 For a Kähler manifold $(\widetilde{M}, \widetilde{g}, \widetilde{I}, \widetilde{\omega})$ with a tensor $\widetilde{\eta}$ in $T^{*} M \otimes T M \otimes T^{*} M$ such that $b_{2} \tilde{\eta}$ is a section of $\llbracket S_{3,0} \widetilde{M} \rrbracket$ and with a connection $\nabla$ with connection form $\omega^{\nabla}=\widetilde{\omega}^{L C}+\widetilde{\eta}$, then

$$
\Omega^{\nabla}=0 \quad \text { if and only if }\left\{\begin{array}{l}
\widetilde{\Omega}^{L C}+\frac{1}{2}[\tilde{\eta} \wedge \tilde{\eta}]=0 \\
\widetilde{d}^{L C} \widetilde{\eta}=0
\end{array} .\right.
$$

\section{Conic and projective special Kähler metrics}

In this section, we will consider the case of a projective special Kähler manifold $(\pi: \widetilde{M} \rightarrow M, \nabla)$ and we will give the explicit relation between the metric on $\widetilde{M}$ and the one on $M$ (see, e.g. [14, Section 1.1]). 
The mapping $\pi: \widetilde{M} \rightarrow M$ is a $\mathbb{C}^{*}$-principal bundle with infinitesimal principal action generated by $\xi$ and $\widetilde{I} \xi$. We can always build the function $r=\sqrt{-\widetilde{g}(\xi, \xi)}: \widetilde{M} \rightarrow \mathbb{R}^{+}$and define $S=r^{-1}(1) \subseteq \widetilde{M}$ with inclusion map $\imath_{S}: S \hookrightarrow \widetilde{M}$. Now $r$ has no critical points, since

$$
\begin{aligned}
d r & =\frac{d\left(r^{2}\right)}{2 r}=\frac{\widetilde{\nabla}^{L C}\left(r^{2}\right)}{2 r}=-\frac{\widetilde{\nabla}^{L C}(\widetilde{g}(\xi, \xi))}{2 r} \\
& =-\frac{2 \widetilde{g}\left(\widetilde{\nabla}^{L C} \xi, \xi\right)}{2 r}=-\frac{\widetilde{g}(\cdot, \xi)}{r}=-\frac{1}{r} \xi^{b}
\end{aligned}
$$

and $\widetilde{g}$ is non-degenerate. It follows that $S$ is a submanifold of dimension $2 n+1$ whose tangent bundle corresponds to $\operatorname{ker}(d r) \subset T \widetilde{M}$. Notice that $\operatorname{dr}(\widetilde{I} \xi)=-\frac{\widetilde{g}(\widetilde{I} \xi, \xi)}{r}=-\frac{\widetilde{\omega}(\xi, \xi)}{r}=0$, so $\widetilde{I} \xi$ is a vector field tangent to $S$ and it induces a principal U(1)-action. The induced metric on $S$ is $g_{S}=l_{S}^{*} \tilde{g}$, and thus, $\mathcal{L}_{\widetilde{I} \xi} g_{S}=l_{S}^{*} \mathcal{L}_{\widetilde{I} \xi} \widetilde{g}=0$.

The principal action of $\mathbb{C}^{*}$ on $\tilde{M}$ induces by inclusion an $\mathbb{R}^{+}$-action, and in addition we have

Lemma 4.1 The map $r: \widetilde{M} \rightarrow \mathbb{R}^{+}$is degree 1 homogeneous with respect to the action of $\mathbb{R}^{+} \subseteq \mathbb{C}^{*}$ on $\widetilde{M}$, i.e. for all $s \in \mathbb{R}^{+}$and $p \in \widetilde{M}$

$$
r(p s)=r(p) s .
$$

As a consequence of this lemma, we can now define a retraction

$$
p: \widetilde{M} \longrightarrow S, \quad u \longmapsto u \frac{1}{r(u)},
$$

which is well defined since $r(p(u))=r\left(u \frac{1}{r(u)}\right)=\frac{r(u)}{r(u)}=1$. Moreover, $p l_{S}=\mathrm{id}_{S}$ implies the surjectivity of $p$, which allows us to see $p: \widetilde{M} \rightarrow S$ as a principal $\mathbb{R}^{+}$-bundle and $\pi_{S}:=\pi l_{S}: S \rightarrow M$ as a principal $S^{1}$-bundle; the composition of the two gives $\pi$.

Lemma 4.2 If $(\pi: \widetilde{M} \rightarrow M, \nabla)$ is projective special Kähler, then $\widetilde{M}$ is diffeomorphic to $S \times \mathbb{R}^{+}$, and moreover

$$
\widetilde{g}=r^{2} p^{*} g_{S}-d r^{2}
$$

Proof Let $a: S \times \mathbb{R}^{+} \rightarrow \tilde{M}$ be the restriction of the principal right action $\tilde{M} \times \mathbb{R}^{+} \rightarrow \tilde{M}$ to $S \times \mathbb{R}^{+}$and consider also $(p, r): \widetilde{M} \rightarrow S \times \mathbb{R}^{+}$. These maps are smooth and each an inverse to the other, in fact if $u \in \widetilde{M}, a(p, r)(u)=a(p(u), r(u))=u \frac{1}{r(u)} r(u)=u$ and for all $(q, s) \in S \times \mathbb{R}^{+},\left(\pi_{S}, r\right) a(q, s)=(p(q s), r(q s))=\left(q \frac{s}{r(q s)}, r(q) s\right)=(q, s)$.

For the second statement, consider the symmetric tensor

$$
g^{\prime}=\frac{1}{r^{2}}\left(\widetilde{g}+d r^{2}\right)
$$

We want to prove it is basic, that is horizontal and invariant with respect to the principal $\mathbb{R}^{+}$ -action.

Since there is only one vertical direction, and since $g^{\prime}$ is symmetric, it is enough to check whether $g^{\prime}$ vanishes when evaluated on the fundamental vector field $\xi$ in one component. Using (2) we obtain 


$$
g^{\prime}(\xi, \cdot)=\frac{1}{r}(\widetilde{g}(\xi, \cdot)+d r(\xi) d r)=\frac{1}{r}(-r d r+r d r)=0 .
$$

And now for the $\mathbb{R}^{+}$-invariance:

$$
\begin{aligned}
\mathcal{L}_{\xi} g^{\prime} & =-2 \frac{\mathcal{L}_{\xi} r}{r^{3}}\left(\widetilde{g}+d r^{2}\right)+\frac{1}{r^{2}}\left(\mathcal{L}_{\xi} \widetilde{g}+2 \mathcal{L}_{\xi}(d r) d r\right) \\
& =-2 \frac{d r(\xi)}{r^{3}}\left(\widetilde{g}+d r^{2}\right)+\frac{1}{r^{2}}\left(2 \widetilde{g}+2\left(d l_{\xi} d r+l_{\xi} d^{2} r\right) d r\right) \\
& =-2 \frac{r}{r^{3}}\left(\widetilde{g}+d r^{2}\right)+\frac{1}{r^{2}}\left(2 \widetilde{g}+2 d r^{2}\right)=0 .
\end{aligned}
$$

Therefore $g^{\prime}$ is basic, which in turn implies it is of the form $p^{*} g^{\prime \prime}$ for some tensor $g^{\prime \prime} \in T_{2} S$, so that

$$
\widetilde{g}=r^{2} p^{*} g^{\prime \prime}-d r^{2}
$$

The proof is ended by the following observation:

$$
g_{S}=\imath_{S}^{*} \tilde{g}=\imath_{S}^{*}\left(r^{2} p^{*} g^{\prime \prime}-d r^{2}\right)=\imath_{S}^{*} p^{*} g^{\prime \prime}-\imath_{S}^{*} d r^{2}=\left(p \imath_{S}\right)^{*} g^{\prime \prime}=g^{\prime \prime}
$$

The $\mathbb{C}^{*}$-bundle $\pi: \widetilde{M} \rightarrow M$ has a unique principal connection orthogonal to the fibres with respect to $\tilde{g}$; the connection form can be written as

$$
\frac{d r}{r}+i \widetilde{\varphi}
$$

Explicitly, we can describe $\tilde{\varphi}$ using the metric:

$$
\widetilde{\varphi}=\frac{\widetilde{g}(\widetilde{I} \xi, \cdot)}{\widetilde{g}(\widetilde{I} \xi, \widetilde{I} \xi)}=-\frac{1}{r^{2}} I \xi^{b}=-\frac{1}{r^{2}} l_{\xi} \widetilde{\omega} .
$$

If we restrict it to $S$, we obtain a connection form $\varphi=l_{S}^{*} \widetilde{\varphi}=-l_{S}^{*}\left(l_{\xi} \omega\right)$ corresponding to the $S^{1}$-action on $S$.

Notice that $p^{*} \varphi=\widetilde{\varphi}$, because the connection form (3) is right-invariant, so $\widetilde{\varphi}=p^{*} \varphi^{\prime}$ for some $\varphi^{\prime}$, and thus, $\varphi=l_{S}^{*} \widetilde{\varphi}=l_{S}^{*} p^{*} \varphi^{\prime}=\left(p t_{S}\right)^{*} \varphi^{\prime}=\varphi^{\prime}$.

The moment map for the action generated by $\widetilde{I} \xi$ is $\mu: \widetilde{M} \rightarrow \mathfrak{t}(1) \cong \mathbb{R}$ such that $d \mu=r_{I \xi} \omega=-\xi^{b}=r d r=d\left(\frac{r^{2}}{2}\right)$, so up to an additive constant, we can assume

$$
\mu=\frac{r^{2}}{2} .
$$

Since $S=\mu^{-1}\left(\frac{1}{2}\right)$ is a level set of the moment map and $M$ is the Kähler quotient, $\pi_{S}: S \rightarrow M$ is a pseudo-Riemannian submersion, and thus, we can write $g_{S}=\pi_{S}^{*} g-\varphi^{2}$.

Proposition 4.3 A projective special Kähler manifold $(\pi: \widetilde{M} \rightarrow M, \nabla)$ satisfies

$$
\begin{aligned}
& \tilde{g}=r^{2} \pi^{*} g-r^{2} \widetilde{\varphi}^{2}-d r^{2}, \\
& \widetilde{\omega}=r^{2} \pi^{*} \omega_{M}+r \widetilde{\varphi} \wedge d r .
\end{aligned}
$$

Proof From the previous arguments 


$$
\begin{aligned}
\widetilde{g} & =r^{2} p^{*} g_{S}-d r^{2}=r^{2} p^{*}\left(\pi_{S}^{*} g-\varphi^{2}\right)-d r^{2} \\
& =r^{2}\left(\pi_{S} p\right)^{*} g-r^{2} \widetilde{\varphi}^{2}-d r^{2}=r^{2} \pi^{*} g-r^{2} \widetilde{\varphi}^{2}-d r^{2} .
\end{aligned}
$$

For the Kähler form, it is enough to notice that $\pi$ is holomorphic, $M$ being a Kähler quotient, and that

$$
(r \widetilde{\varphi}) \circ \widetilde{I}=-\frac{1}{r} \widetilde{I} \xi^{\widetilde{I}} \widetilde{I}=-\frac{1}{r} \xi^{b}=d r .
$$

For future reference, we give the following

Remark 4.4 The curvature of $\varphi$ is computed using Lemma 2.2:

$$
d \varphi=-d l_{S}^{*} l_{\xi} \widetilde{\omega}=l_{S}^{*}\left(-\mathcal{L}_{\xi} \widetilde{\omega}+l_{\xi} d \widetilde{\omega}\right)=-2 l_{S}^{*} \widetilde{\omega}=-2 \pi_{S}^{*} \omega_{M} .
$$

in fact, the restriction to $S$ of $\widetilde{\omega}$ maps fixes $r=1$ and thus kills $d r$.

It will be useful to compute also

$$
d \widetilde{\varphi}=-2 \pi^{*} \omega_{M}
$$

\section{Lifting the coframe}

The purpose of this section is to lift a generic unitary coframe on a projective special Kähler manifold to one on the corresponding conic special Kähler. This will enable us to give a more explicit formulation of the Levi-Civita connection and associated curvature tensor on the conic special Kähler manifold.

In our convention, on a Kähler manifold $(M, g, I, \omega)$, the Hermitian form is $h=g+i \omega$. Given a projective special Kähler manifold $(\pi: \widetilde{M} \rightarrow M, \nabla)$ and an open subset $U \subseteq M$, consider a unitary coframe $\theta=\left(\theta^{1}, \ldots, \theta^{n}\right) \in \Omega^{1}\left(U, \mathbb{C}^{n}\right)$ on $M$, then we can build a coframe $\widetilde{\theta} \in \Omega^{1}\left(\pi^{-1}(U), \mathbb{C}^{n+1}\right)$ on $\widetilde{M}$ as follows:

$$
\widetilde{\theta}^{k}=\left\{\begin{array}{ll}
r \pi^{*} \theta^{k} & \text { if } k \leq n \\
d r+i r \tilde{\varphi} & \text { if } k=n+1
\end{array} .\right.
$$

This coframe is compatible with the $\mathrm{U}(n, 1)$-structure because it takes complex values and

$$
\sum_{k=1}^{n} \overline{\widetilde{\theta}^{k}} \widetilde{\theta}^{k}-\overline{\widetilde{\theta}^{n+1}} \widetilde{\theta}^{n+1}=r^{2} \pi^{*}\left(\sum_{k=1}^{n} \overline{\theta^{k}} \theta^{k}\right)-d r^{2}-r^{2} \widetilde{\varphi}^{2}=\widetilde{g}
$$

We will denote the dual frame to a given coframe by the same symbol, but with lower indices.

Remark 5.1 Let $T=\mathbb{C}^{n+1}$ be the standard real representation of $\mathrm{U}(n, 1)$, and let $T \otimes_{\mathbb{R}} \mathbb{C} \cong T^{1,0} \oplus T^{0,1}$ be the holomorphic, anti-holomorphic split. Given a connection on a Kähler manifold, it can be represented by a connection form $\omega$ with values in $\mathfrak{t}(n, 1)$ whose complexification is $\mathfrak{g l}(n+1, \mathbb{C}) \cong T^{1,0} \otimes T_{1,0} \oplus T^{0,1} \otimes T_{1,0}$, so we obtain 
projections in each component, respectively $\omega_{1,0}^{1,0}$ and $\omega_{0,1}^{0,1}$ such that $\omega=\omega_{1,0}^{1,0}+\omega_{0,1}^{0,1}$. Notice that $\omega_{0,1}^{0,1}=\overline{\omega_{1,0}^{1,0}}$ because $\omega$ comes from a real representation and to give the first component is equivalent to give the whole form. Notice also that ( $\llbracket T \rrbracket, I)$, as complex representation, is isomorphic to $T^{1,0}$ and the component $A_{1,0}^{1,0}$ of an endomorphism $A$ gives the corresponding endomorphism of $T^{1,0}$. We will often present connection forms by giving only the $T_{1,0}^{1,0}$ component.

We will call $\Re$ the projection from the complex tensor algebra to the real representation, defined so that $\Re(\alpha)=\alpha+\bar{\alpha}$ where the conjugate is the real structure.

Proposition 5.2 Let $(\pi: \widetilde{M} \rightarrow M, \nabla)$ be a projective special Kähler manifold, let $(U, \theta)$ be a local unitary coframe on $M$ lifted as in (4) to a coframe $\widetilde{\theta}$ adapted to the $\mathrm{U}(n, 1)$-structure on $\widetilde{M}$. With respect to $\widetilde{\theta}$, the Levi-Civita connection form on $\widetilde{M}$ is represented by

$$
\widetilde{\omega}^{L C}=\left(\begin{array}{c|c}
\pi^{*} \omega^{L C} & 0 \\
\hline 0 & 0
\end{array}\right)+\frac{1}{r}\left(\begin{array}{ccc|c}
i \operatorname{Im}\left(\widetilde{\theta}^{n+1}\right) & & 0 & \widetilde{\theta}^{1} \\
& \ddots & & \vdots \\
0 & & i \operatorname{Im}\left(\widetilde{\theta}^{n+1}\right) & \widetilde{\theta}^{n} \\
\hline \widetilde{\tilde{\theta}^{1}} & \ldots & \widetilde{\widetilde{\theta}^{n}} & i \operatorname{Im}\left(\widetilde{\theta}^{n+1}\right)
\end{array}\right),
$$

that is

$$
\widetilde{\omega}^{L C}=\left(\begin{array}{c|c}
\pi^{*} \omega^{L C}+i \widetilde{\varphi} \otimes I_{n} & \pi^{*} \theta \\
\hline \pi^{*} \theta^{\star} & i \widetilde{\varphi}
\end{array}\right)
$$

and its curvature form is

$$
\widetilde{\Omega}^{L C}=\left(\begin{array}{c|c}
\pi^{*}\left(\Omega^{L C}+\theta \wedge \theta^{*}-2 i \omega_{M} \otimes \mathrm{id}\right) & 0 \\
\hline 0 & 0
\end{array}\right) .
$$

Proof The connection form (5) is metric if and only if the matrix is anti-Hermitian with respect to $\widetilde{g}$ and since $\omega^{L C}$ is anti-Hermitian with respect to $g$, we get

$$
\left(\widetilde{\omega}^{L C}\right)^{\star}=\left(\begin{array}{c|c}
\pi^{*}\left(\omega^{L C}\right)^{\star}-i \widetilde{\varphi} \otimes I_{n} & -\pi^{*} \theta \\
\hline-\pi^{*} \theta^{\star} & -i \widetilde{\varphi}
\end{array}\right)=-\widetilde{\omega}^{L C} .
$$

The torsion form of this connection is $\widetilde{\Theta}^{L C}=d \widetilde{\theta}+\widetilde{\omega}^{L C} \wedge \widetilde{\theta}$, so for $1 \leq k \leq n$

$$
\begin{aligned}
\left(\widetilde{\Theta}^{L C}\right)^{k} & =d \widetilde{\theta}^{k}+\sum_{j=1}^{n}\left(\widetilde{\omega}^{L C}\right)_{j}^{k} \wedge \widetilde{\theta}^{j}+\left(\widetilde{\omega}^{L C}\right)_{n+1}^{k} \wedge \widetilde{\theta}^{n+1} \\
& =d\left(r \pi^{*} \theta^{k}\right)+\sum_{j=1}^{n}\left(\pi^{*}\left(\omega^{L C}\right)_{j}^{k}+i \widetilde{\varphi} \delta_{j}^{k}\right) \wedge\left(r \pi^{*} \theta^{j}\right)+\pi^{*} \theta^{k} \wedge \widetilde{\theta}^{n+1} \\
& =r \pi^{*}\left(\Theta^{L C}\right)^{k}+(d r+i r \widetilde{\varphi}) \wedge \pi^{*} \theta^{k}+\pi^{*} \theta^{k} \wedge \widetilde{\theta}^{n+1} \\
& =0+\widetilde{\theta}^{n+1} \wedge \pi^{*} \theta^{k}+\pi^{*} \theta^{k} \wedge \widetilde{\theta}^{n+1}=0 .
\end{aligned}
$$

In the last component 


$$
\begin{aligned}
\left(\widetilde{\Theta}^{L C}\right)^{n+1} & =d \widetilde{\theta}^{n+1}+\sum_{j=1}^{n} \pi^{*} \overline{\theta^{j}} \wedge r \pi^{*} \theta^{j}+i \widetilde{\varphi} \wedge \widetilde{\theta}^{n+1} \\
& =d(d r+i r \widetilde{\varphi})+r \pi^{*}\left(\sum_{j=1}^{n} \overline{\theta^{j}} \wedge \theta^{j}\right)+i \widetilde{\varphi} \wedge \widetilde{\theta}^{n+1} \\
& =i d r \wedge \widetilde{\varphi}+i r\left(d \widetilde{\varphi}+2 \pi^{*} \omega_{M}\right)+i \widetilde{\varphi} \wedge d r=0 .
\end{aligned}
$$

$\widetilde{\omega}^{L C}$ is metric and torsion-free; therefore by uniqueness, it must be the Levi-Civita connection.

Now let us compute its curvature form $\widetilde{\Omega}^{L C}=d \widetilde{\omega}^{L C}+\widetilde{\omega}^{L C} \wedge \widetilde{\omega}^{L C}$. For $1 \leq k, h \leq n$ we have

$$
\begin{aligned}
\left(\widetilde{\Omega}^{L C}\right)_{k}^{h}= & d\left(\widetilde{\omega}^{L C}\right)_{k}^{h}+\left(\widetilde{\omega}^{L C}\right)_{j}^{h} \wedge\left(\widetilde{\omega}^{L C}\right)_{k}^{j} \\
= & d \pi^{*}\left(\omega^{L C}\right)_{k}^{h}+i d \widetilde{\varphi} \delta_{k}^{h}+\sum_{j=1}^{n}\left(\pi^{*}\left(\omega^{L C}\right)_{j}^{h}+i \widetilde{\varphi} \delta_{j}^{h}\right) \wedge\left(\pi^{*}\left(\omega^{L C}\right)_{k}^{j}+i \widetilde{\varphi} \delta_{k}^{j}\right)+\pi^{*} \theta^{h} \wedge \pi^{*} \bar{\theta}^{k} \\
= & \pi^{*} d\left(\omega^{L C}\right)_{k}^{h}-2 i \pi^{*} \omega_{M} \delta_{k}^{h}+\pi^{*}\left(\left(\omega^{L C}\right)_{j}^{h} \wedge\left(\omega^{L C}\right)_{k}^{j}\right. \\
& +i \widetilde{\varphi} \wedge \pi^{*}\left(\omega^{L C}\right)_{k}^{h}+\pi^{*}\left(\omega^{L C}\right)_{k}^{h} \wedge i \widetilde{\varphi}-\widetilde{\varphi} \wedge \widetilde{\varphi} \delta_{k}^{h}+\pi^{*} \theta^{h} \wedge \pi^{*} \bar{\theta}^{k} \\
= & \pi^{*}\left(\Omega^{L C}\right)_{k}^{h}-2 i \pi^{*} \omega_{M} \delta_{k}^{h}+\pi^{*}\left(\theta^{h} \wedge \overline{\theta^{k}}\right)
\end{aligned}
$$

and

$$
\left(\widetilde{\Omega}^{L C}\right)_{n+1}^{h}=d \pi^{*} \theta^{h}+\sum_{j=1}^{n}\left(\pi^{*}\left(\omega^{L C}\right)_{j}^{h}+i \widetilde{\varphi} \delta_{j}^{h}\right) \wedge \pi^{*} \theta^{j}+\pi^{*} \theta^{h} \wedge i \widetilde{\varphi}=\pi^{*}\left(\Theta^{L C}\right)^{h}=0 .
$$

Since the curvature form must also be anti-Hermitian, we also get

$$
\left(\widetilde{\Omega}^{L C}\right)_{k}^{n+1}=-\left(\left(\widetilde{\Omega}^{L C}\right)^{\star}\right)_{k}^{n+1}=\overline{\left(\widetilde{\Omega}^{L C}\right)_{n+1}^{k}}=0 .
$$

Finally,

$$
\left(\widetilde{\Omega}^{L C}\right)_{n+1}^{n+1}=i d \widetilde{\varphi}+\sum_{j=1}^{n} \pi^{*} \overline{\theta^{j}} \wedge \pi^{*} \theta^{j}-\widetilde{\varphi} \wedge \widetilde{\varphi}=i d \widetilde{\varphi}+2 i \pi^{*} \omega_{M}=0
$$

Remark 5.3 The tensor $\theta \wedge \theta^{\star}-2 i \omega_{M} \otimes i d$, or explicitly

$$
\Omega_{\mathbb{P}_{\mathrm{C}}^{n}}:=\Re\left(\left(\theta^{k} \wedge \overline{\theta^{h}}\right) \otimes \theta_{k} \otimes \theta^{h}-\left(\overline{\theta^{k}} \wedge \theta^{k}\right) \otimes \theta_{h} \otimes \theta^{h}\right)
$$

is a curvature tensor of the complex projective space of dimension $n$; in fact, $\Omega_{\mathbb{P}_{C}^{n}}$ is the curvature with respect to the Fubini-Study metric (see for example [24, II, p. 277]). In order to verify that $\Omega_{\mathbb{P}_{C}^{n}}$ is exactly the curvature of the Fubini-Study rather than a multiple, we compute the Ricci tensor: 


$$
\operatorname{Ric}_{\mathbb{P}_{\mathrm{C}}^{n}}=\Re\left(n \theta^{h} \otimes \overline{\theta^{h}}+\delta_{h, k} \theta^{h} \otimes \overline{\theta^{k}}\right)=\Re((n+1) h)=2(n+1) g .
$$

Then,

$$
\operatorname{scal}_{\mathbb{P}_{\mathrm{C}}^{n}}=2(n+1) .
$$

Thus $\Omega_{\mathbb{P}_{C}^{n}}$ corresponds exactly to the curvature of $\mathbb{P}_{\mathbb{C}}^{n}$ with the Fubini-Study metric.

Now, whenever we have a smooth map $f: M \rightarrow N$ between Riemannian manifolds, we can extend the pull-back $f^{*}: T . N \rightarrow T . M$ on the covariant tensor algebra to the whole tensor algebra, using the musical isomorphisms in each contravariant component. Explicitly, for $X$ vector field on $N$, we define $f^{*} X:=\sharp f^{*} b X=\left(f^{*} X^{b}\right)_{\sharp}$. Notice that this extension of the pull-back is still functorial, since if $f: M \rightarrow N, g: N \rightarrow L$ are smooth maps, then $f^{*} g^{*} X=\sharp f^{*} b \sharp g^{*} b X=\sharp f^{*} g^{*} b X=\sharp(g f)^{*} b X=(g f)^{*} X$.

Since $\widetilde{M}$ and $M$ are Riemannian manifolds, we have $\pi^{*}: T_{\bullet}^{*} M \rightarrow T_{\bullet}^{*} \widetilde{M}$, and in particular, for $1 \leq k \leq n$ we have

$$
\pi^{*} \theta_{k}=\left(\pi^{*} \theta_{k}^{b}\right)_{\sharp}=\frac{1}{2}\left(\pi^{*} \overline{\theta^{k}}\right)_{\sharp}=\frac{1}{2 r}\left(\overline{\widetilde{\theta}^{k}}\right)_{\sharp}=\frac{1}{r} \widetilde{\theta}_{k} .
$$

Remark 5.4 In this notation,

$$
\widetilde{\Omega}^{L C}=r^{2} \pi^{*}\left(\Omega^{L C}+\Omega_{\mathbb{P}_{\mathbb{C}}^{n}}\right)
$$

\section{Deviance}

In this section, we will continue the analysis of the tensor $\tilde{\eta}$ started in section 3 . The aim is to reduce it to a locally defined tensor on $M$ that we call deviance. We will then use it to give an explicit local description of the Ricci tensor and the scalar curvature.

Lemma 6.1 On a projective special Kähler manifold $(\pi: \tilde{M} \rightarrow M, \nabla)$, if $\tilde{\eta}_{X} Y=\nabla_{X} Y-\widetilde{\nabla}_{X}^{L C} Y$, then $b_{2} \tilde{\eta}$ is horizontal with respect to $\pi$.

In other words, $b_{2}(\widetilde{\eta})$ is a section of $\pi^{*} \llbracket S_{3,0} M \rrbracket \subset \llbracket S_{3,0} \tilde{M} \rrbracket$. Explicitly, $\tilde{\eta}_{v}, \tilde{\eta} v$ and $\widetilde{g}(\widetilde{\eta}, v)$ vanish for all $v \in\langle\xi, I \xi\rangle$.

Proof First notice that $\widetilde{\eta}(\xi)=\nabla \xi-\widetilde{\nabla}^{L C} \xi=0$, so by symmetry $\tilde{\eta}_{\xi}=0$ and $g(\eta, \xi)=0$, so $b_{2}(\widetilde{\eta})$ in each component vanishes when evaluated at $\xi$. From this fact and (1), we also deduce $\widetilde{\eta}(\widetilde{I} \xi)=\widetilde{I} \widetilde{\eta}(\xi)+[\widetilde{\eta}, \widetilde{I}] \xi=0-2 \widetilde{I} \widetilde{\eta}(\xi)=0$. By symmetry, we conclude that $b_{2} \widetilde{\eta}$ vanishes in every component on $I \xi$. Linearity then completes the proof.

Lemma 6.2 Let $(\tilde{M}, \widetilde{g}, \widetilde{I}, \widetilde{\omega}, \nabla, \xi)$ be a conic special Kähler manifold and $\tilde{\eta}$ be as above, then

1. $\mathcal{L}_{\xi} \tilde{\eta}=0$

2. $\mathcal{L}_{\tilde{I} \xi} \tilde{\eta}=-2 \widetilde{I} \tilde{\eta}$. 
Proof The proof relies on a generic formula satisfied by a torsion-free connection $D$ (see, e.g. [26, equation (3.1), p. 1336]), that is:

$$
\mathcal{L}_{A}\left(D_{X} Y\right)-D_{\mathcal{L}_{A} X} Y-D_{X} \mathcal{L}_{A} Y=\Omega^{D}(A, X) Y-D_{D_{X} Y} A+D_{X} D_{Y} A
$$

1. We check the formula on vector fields $X, Y \in \mathfrak{X}(\tilde{M})$

$$
\begin{aligned}
\left(\mathcal{L}_{\xi} \widetilde{\eta}\right)_{X} Y= & \mathcal{L}_{\xi}\left(\widetilde{\eta}_{X} Y\right)-\widetilde{\eta}_{\mathcal{L}_{\xi} X} Y-\widetilde{\eta}_{X} \mathcal{L}_{\xi} Y \\
= & \mathcal{L}_{\xi} \nabla_{X} Y-\mathcal{L}_{\xi} \widetilde{\nabla}_{X}^{L C} Y-\nabla_{\mathcal{L}_{\xi} X} Y+\widetilde{\nabla}_{\mathcal{L}_{\xi} X}^{L C} Y \\
& -\nabla_{X} \mathcal{L}_{\xi} Y+\widetilde{\nabla}_{X}^{L C} \mathcal{L}_{\xi} Y \\
= & \Omega^{\nabla}(\xi, X) Y-\nabla_{\nabla_{X} Y} \xi+\nabla_{X} \nabla_{Y} \xi-\widetilde{\Omega}^{L C}(\xi, X) Y \\
& +\widetilde{\nabla}_{\widetilde{\nabla}_{X}^{L C} Y}^{L C} \xi-\widetilde{\nabla}_{X}^{L C} \widetilde{\nabla}_{Y}^{L C} \xi \\
= & -\nabla_{X} Y+\nabla_{X} Y-\widetilde{\Omega}^{L C}(\xi, X) Y+\widetilde{\nabla}_{X}^{L C} Y-\widetilde{\nabla}_{X}^{L C} Y \\
= & -\widetilde{\Omega}^{L C}(\xi, X) Y .
\end{aligned}
$$

Lowering the contravariant index of the curvature form, for $Z \in \mathfrak{X}(\widetilde{M})$, thanks to the symmetries of the Riemannian tensor we obtain

$$
\begin{aligned}
\widetilde{g}\left(\widetilde{\Omega}^{L C}(\xi, X) Y, Z\right) & =\widetilde{g}\left(\widetilde{\Omega}^{L C}(Y, Z) \xi, X\right) \\
& =\widetilde{g}\left(\widetilde{\nabla}_{Y}^{L C} \widetilde{\nabla}_{Z}^{L C} \xi-\widetilde{\nabla}_{Z}^{L C} \widetilde{\nabla}_{Y}^{L C} \xi-\widetilde{\nabla}_{[Y, Z]}^{L C} \xi, X\right) \\
& =\widetilde{g}\left(\widetilde{\nabla}_{Y}^{L C} Z-\widetilde{\nabla}_{Z}^{L C} Y-[Y, Z], X\right) \\
& =\widetilde{g}\left(\Theta^{L C}(Y, Z), X\right)=0,
\end{aligned}
$$

proving that $\widetilde{\Omega}^{L C}(\xi, X) Y=0$, which implies the statement.

2. As before,

$$
\begin{aligned}
\left(\mathcal{L}_{\widetilde{I} \xi} \widetilde{\eta}\right)_{X} Y= & \Omega^{\nabla}(\widetilde{I} \xi, X) Y-\nabla_{\nabla_{X} Y}(\widetilde{I} \xi)+\nabla_{X} \nabla_{Y}(\widetilde{I} \xi)-\widetilde{\Omega}^{L C}(\widetilde{I} \xi, X) Y \\
& +\widetilde{\nabla}_{\widetilde{\nabla}_{X}^{L C} Y}^{L C}(\widetilde{I} \xi)-\widetilde{\nabla}_{X}^{L C} \widetilde{\nabla}_{Y}^{L C}(\widetilde{I} \xi) \\
= & -\widetilde{I} \nabla_{X} Y+\nabla_{X}(\widetilde{I} Y)-\widetilde{\Omega}^{L C}(\widetilde{I} \xi, X) Y+\widetilde{I}_{X}^{L C} Y-\widetilde{\nabla}_{X}^{L C}(\widetilde{I} Y) \\
= & (\nabla \widetilde{I})(X, Y)-\widetilde{\Omega}^{L C}(\widetilde{I} \xi, X) Y .
\end{aligned}
$$

Proceeding as in the previous point 


$$
\begin{aligned}
& \tilde{g}\left(\widetilde{\Omega}^{L C}(\widetilde{I} \xi, X) Y, Z\right)=\widetilde{g}\left(\widetilde{\Omega}^{L C}(Y, Z)(\widetilde{I} \xi), X\right) \\
& \quad=\widetilde{g}\left(\widetilde{\nabla}_{Y}^{L C} \widetilde{\nabla}_{Z}^{L C}(\widetilde{I} \xi)-\widetilde{\nabla}_{Z}^{L C} \widetilde{\nabla}_{Y}^{L C}(\widetilde{I} \xi)-\widetilde{\nabla}_{[Y, Z]}^{L C}(\widetilde{I} \xi), X\right) \\
& \quad=\widetilde{g}\left(\widetilde{I} \widetilde{\Omega}^{L C}(Y, Z) \xi, X\right)=-\widetilde{g}\left(\widetilde{\Omega}^{L C}(Y, Z) \xi, I X\right) \\
& \quad=-\widetilde{g}\left(\widetilde{\Omega}^{L C}(\xi, \widetilde{I} X) Y, Z\right) .
\end{aligned}
$$

This quantity is zero as shown in the previous point, so it follows that $\mathcal{L}_{\widetilde{I} \xi} \tilde{\eta}=\nabla \widetilde{I}$, so (1) ends the proof.

We can now use a coframe $\widetilde{\theta}$ as in section 5 in order to progress in the study of $\widetilde{\eta}$. We then write

$$
\widetilde{\eta}=\Re\left(\widetilde{\eta}_{k, h}^{j} \widetilde{\theta}^{k} \otimes \widetilde{\widetilde{\theta}}_{j} \otimes \widetilde{\theta}^{h}\right)
$$

Since every operator we use is $\mathbb{C}$-linear, we can study only the component in $T_{1,0} \otimes T^{0,1} \otimes T_{1,0}$, that is $\widetilde{\eta}_{k, h}^{j} \widetilde{\theta}^{k} \otimes \widetilde{\theta}_{j} \otimes \widetilde{\theta}^{h}$. Because of Lemma 6.1 , the coefficients $\widetilde{\eta}_{k, h}^{j}$ vanish if any one of the indices is $n+1$; moreover, $\widetilde{\eta}_{k, h}^{j}$ is completely symmetric in its indices. The last statement follows from the fact that $b_{2} \widetilde{\eta}$ is a tensor in $\pi^{*} S_{3,0} M$, and such tensors are expressed using only $\pi^{*} \theta^{k}$ for $1 \leq k \leq n$, where the metric is positive definite, and thus, $b_{2}$ does not change the signs of the coefficients of $\tilde{\eta}$.

We are now ready to reduce $\tilde{\eta}$ to an object defined locally on the base space.

Proposition 6.3 Given a projective special Kähler $(\pi: \tilde{M} \rightarrow M, \nabla)$ and a section $s: U \rightarrow S \subseteq \widetilde{M}$ inducing a trivialisation $\left(\left.\pi\right|_{\pi^{-1}(U)}, z\right): \pi^{-1}(U) \rightarrow U \times \mathbb{C}^{*}$, there exists a tensor $\eta$ in $T_{1,0} U \otimes T^{0,1} U \otimes T_{1,0} U$ such that $b_{2} \eta$ is a tensor in $S_{3,0} U$ and

$$
\tilde{\eta}=\Re\left(z^{2} \pi^{*} \eta\right)=r^{2} \cos (2 \vartheta) 2 \operatorname{Re} \pi^{*} \eta+r^{2} \sin (2 \vartheta) 2 \operatorname{Im} \pi^{*} \eta
$$

where $z=r e^{i 9}$.

Proof For every point $p \in M$, we can find a local unitary coframe $\theta$ defined on an open set containing $p$, and the corresponding coframe $\widetilde{\theta}$ on $\widetilde{M}$ as in (4).

For the coming arguments, we first compute the following Lie derivatives

$$
\begin{aligned}
& \mathcal{L}_{\xi} \widetilde{\theta^{k}}=d l_{\xi}\left(r \pi^{*} \theta^{k}\right)+l_{\xi} d\left(r \pi^{*} \theta^{k}\right)=0+l_{\xi}\left(d r \wedge \pi^{*} \theta^{k}\right)+r l_{\xi} d \pi^{*} \theta^{k} \\
& =d r(\xi) \pi^{*} \theta^{k}+r l_{\xi} \pi^{*} d \theta^{k}=r \pi^{*} \theta^{k}+0=\widetilde{\theta}^{k} ; \\
& \mathcal{L}_{\xi} \widetilde{\theta}_{k}=\widetilde{g}\left(\mathcal{L}_{\xi} \widetilde{\theta}_{k}, \cdot\right)_{\sharp}=\mathcal{L}_{\xi}\left(\tilde{g}\left(\tilde{\theta}_{k}, \cdot\right)\right)_{\sharp}-\left(\mathcal{L}_{\xi} \tilde{g}\left(\widetilde{\theta}_{k}, \cdot\right)\right)_{\sharp} \\
& =\frac{1}{2}\left(\mathcal{L}_{\xi} \overline{\widetilde{\theta}^{k}}\right)_{\sharp}-2 \widetilde{g}\left(\widetilde{\theta}_{k}, \cdot\right)_{\sharp}=\frac{1}{2}{\overline{\theta^{k}}}_{\sharp}-2 \widetilde{\theta}_{k}=-\widetilde{\theta}_{k} ; \\
& \mathcal{L}_{\widetilde{I} \xi} \widetilde{\theta}^{k}=d \tau_{\tau_{\xi}} \widetilde{\theta}^{k}+\tau_{\tau_{\xi}} d \widetilde{\theta}^{k}=d \tau_{\tau_{\xi}}\left(r \pi^{*} \theta^{k}\right)+\tau_{\widetilde{I} \xi} d\left(r \pi^{*} \theta^{k}\right) \\
& =0+r \tau_{I \xi} d \pi^{*} \theta^{k}=r \tau_{I \xi} \pi^{*} d \theta^{k}=0 ; \\
& \mathcal{L}_{\widetilde{I} \xi} \widetilde{\theta}_{k}=\widetilde{g}\left(\mathcal{L}_{\widetilde{I} \xi} \widetilde{\theta}_{k}, \cdot\right)_{\sharp}=\mathcal{L}_{\widetilde{I} \xi}\left(\widetilde{g}\left(\widetilde{\theta}_{k}, \cdot\right)\right)_{\sharp}=\frac{1}{2}\left(\mathcal{L}_{\xi} \overline{\widetilde{\theta}^{k}}\right)_{\sharp}=0 .
\end{aligned}
$$


Lemma 6.2 implies

$$
\begin{aligned}
0=\mathcal{L}_{\xi} \tilde{\eta}= & \mathcal{L}_{\xi} \Re\left(\widetilde{\eta}_{k, h}^{j} \widetilde{\theta}^{k} \otimes \overline{\widetilde{\theta}}_{j} \otimes \widetilde{\theta}^{h}\right) \\
= & \Re\left(\mathcal{L}_{\xi} \widetilde{\eta}_{k, h}^{j} \widetilde{\theta}^{k} \otimes \overline{\widetilde{\theta}}_{j} \otimes \widetilde{\theta}^{h}+\widetilde{\eta}_{k, h}^{j} \mathcal{L}_{\xi} \widetilde{\theta}^{k} \otimes \widetilde{\widetilde{\theta}}_{j} \otimes \widetilde{\theta}^{h}\right. \\
& \left.+\widetilde{\eta}_{k, h}^{j} \widetilde{\theta}^{k} \otimes \mathcal{L}_{\xi} \overline{\widetilde{\theta}}_{j} \otimes \widetilde{\theta}^{h}+\widetilde{\eta}_{k, h}^{j} \widetilde{\theta}^{k} \otimes \overline{\widetilde{\theta}}_{j} \otimes \mathcal{L}_{\xi} \widetilde{\theta}^{h}\right) \\
= & \Re\left(\mathcal{L}_{\xi} \widetilde{\eta}_{k, h}^{j} \widetilde{\theta}^{k} \otimes \overline{\widetilde{\theta}}_{j} \otimes \widetilde{\theta}^{h}+\widetilde{\eta}_{k, h}^{j} \widetilde{\theta}^{k} \otimes \overline{\widetilde{\theta}}_{j} \otimes \widetilde{\theta}^{h}\right) \\
= & \Re\left(\left(\mathcal{L}_{\xi} \tilde{\eta}_{k, h}^{j}+\widetilde{\eta}_{k, h}^{j}\right) \widetilde{\theta}^{k} \otimes \overline{\widetilde{\theta}}_{j} \otimes \widetilde{\theta}^{h}\right) .
\end{aligned}
$$

and

$$
\begin{aligned}
0=\mathcal{L}_{\widetilde{I} \xi} \widetilde{\eta}+2 \widetilde{I} \widetilde{\eta} & =\mathcal{L}_{\widetilde{I}_{\xi}} \Re\left(\widetilde{\eta}_{k, h}^{j} \widetilde{\theta}^{k} \otimes \overline{\widetilde{\theta}}_{j} \otimes \widetilde{\theta}^{h}\right)+\Re\left(2 \widetilde{\eta}_{k, h}^{j} \widetilde{\theta}^{k} \otimes \widetilde{I}\left(\overline{\widetilde{\theta}}_{j}\right) \otimes \widetilde{\theta}^{h}\right) \\
& =\Re\left(\mathcal{L}_{\xi} \widetilde{\eta}_{k, h}^{j} \widetilde{\theta}^{k} \otimes \overline{\widetilde{\theta}}_{j} \otimes \widetilde{\theta}^{h}-2 i \widetilde{\eta}_{k, h}^{j} \widetilde{\theta}^{k} \otimes \overline{\widetilde{\theta}}_{j} \otimes \widetilde{\theta}^{h}\right) \\
& =\Re\left(\left(\mathcal{L}_{I \xi} \widetilde{\eta}_{k, h}^{j}-2 i \widetilde{\eta}_{k, h}^{j}\right) \widetilde{\theta}^{k} \otimes \overline{\widetilde{\theta}}_{j} \otimes \widetilde{\theta}^{h}\right) .
\end{aligned}
$$

Independent components must vanish, so we obtain a family of differential equations for $1 \leq j, k, h \leq n$

$$
\left\{\begin{array}{l}
\mathcal{L}_{\xi} \widetilde{\eta}_{k, h}^{j}=-\widetilde{\eta}_{k, h}^{j} \\
\mathcal{L}_{\widetilde{I} \xi} \widetilde{\eta}_{k, h}^{j}=2 i \widetilde{\eta}_{k, h}^{j}
\end{array} .\right.
$$

We define $\eta$, as the component in $T_{1,0} M \otimes T^{0,1} M \otimes T_{1,0} M$ of $s^{*} \tilde{\eta}$, so that $\Re(\eta)=s^{*} \tilde{\eta}$.

Notice that since $\pi s=\mathrm{id}_{M}$, the pullbacks satisfy $s^{*} \pi^{*}=\mathrm{id}_{T: M}$, so

$$
\begin{aligned}
s^{*} \tilde{\eta} & =s^{*} \mathfrak{R}\left(\widetilde{\eta}_{k, h}^{j} \widetilde{\theta}^{k} \otimes \overline{\widetilde{\theta}}_{j} \otimes \widetilde{\theta}^{h}\right)=\Re\left(s^{*}\left(r^{3} \widetilde{\eta}_{k, h}^{j} \pi^{*} \theta^{k} \otimes \pi^{*} \overline{\theta_{j}} \otimes \pi^{*} \theta^{h}\right)\right) \\
& =\mathfrak{R}\left((r \circ s)^{3}\left(\widetilde{\eta}_{k, h}^{j} \circ s\right) s^{*} \pi^{*} \theta^{k} \otimes s^{*} \pi^{*} \bar{\theta}_{j} \otimes s^{*} \pi^{*} \theta^{h}\right) \\
& =\mathfrak{R}\left(\left(\widetilde{\eta}_{k, h}^{j} \circ s\right) \theta^{k} \otimes \bar{\theta}_{j} \otimes \theta^{h}\right) .
\end{aligned}
$$

Thus $\eta=s^{*} \widetilde{\eta}_{k, h}^{j} \theta^{k} \otimes \overline{\theta_{j}} \otimes \theta^{h}$ and we define $\eta_{k, h}^{j}:=s^{*} \widetilde{\eta}_{k, h}^{j}$.

Now we will use (7) to find $\widetilde{\eta}_{k, h}^{j}$ at a point of $\pi^{*} U$. We define the function $f: \mathbb{R} \rightarrow \mathbb{C}$ such that $f(t):=\tilde{\eta}_{k, h}^{j}\left(s(u) e^{t}\right)$ for $u \in U$ and compute its derivative at $t_{0} \in \mathbb{R}$.

$$
\begin{aligned}
\left.\frac{d}{d t} f\right|_{t_{0}} & =\left.\frac{d}{d t} \widetilde{\eta}_{k, h}^{j}\left(s(u) e^{t}\right)\right|_{t=t_{0}}=\left.\frac{d}{d t} \widetilde{\eta}_{k, h}^{j}\left(s(u) e^{t_{0}+t}\right)\right|_{t=0}=\left.\frac{d}{d t} \widetilde{\eta}_{k, h}^{j}\left(\phi_{\xi}^{t}\left(s(u) e^{t_{0}}\right)\right)\right|_{t=0} \\
& =\left(\mathcal{L}_{\xi} \widetilde{\eta}_{k, h}^{j}\right)\left(s(u) e^{t_{0}}\right)=-\widetilde{\eta}_{k, h}^{j}\left(s(u) e^{t_{0}}\right)=-f\left(t_{0}\right) .
\end{aligned}
$$

Moreover, $f(0)=\widetilde{\eta}_{k, h}^{j}(s(u))=\eta_{k, h}^{j}(u)$, so $f$ satisfies the following initial value problem 


$$
\left\{\begin{array}{l}
f^{\prime}=-f \\
f(0)=\eta_{k, h}^{j}(u)
\end{array}\right.
$$

which has a unique solution, that is $f(t)=\eta_{k, h}^{j}(u) e^{-t}$. This means that $\widetilde{\eta}_{k, h}^{j}\left(s(u) e^{t}\right)=\eta_{k, h}^{j}(u) e^{-t}$ or equivalently, for all $\rho \in \mathbb{R}^{+}$we have $\widetilde{\eta}_{k, h}^{j}(s(u) \rho)=\frac{1}{\rho} \eta_{k, h}^{j}(u)=\left(\frac{1}{r} \pi^{*} \eta_{k, h}^{j}\right)(s(u) \rho)$.

Similarly, consider the function $f: \mathbb{R} \rightarrow \mathbb{C}$ such that $f(t):=\widetilde{\eta}_{k, h}^{j}\left(s(u) \rho e^{i t}\right)$ and compute its derivative at $t_{0} \in \mathbb{R}$.

$$
\begin{aligned}
\left.\frac{d}{d t} f\right|_{t_{0}} & =\left.\frac{d}{d t} \widetilde{\eta}_{k, h}^{j}\left(s(u) \rho e^{i t}\right)\right|_{t=t_{0}}=\left.\frac{d}{d t} \widetilde{\eta}_{k, h}^{j}\left(s(u) \rho e^{i t_{0}+i t}\right)\right|_{t=0} \\
& =\left.\frac{d}{d t} \widetilde{\eta}_{k, h}^{j}\left(\phi_{I \xi}^{t}\left(s(u) \rho e^{i t_{0}}\right)\right)\right|_{t=0}=\left(\mathcal{L}_{I \xi} \widetilde{\eta}_{k, h}^{j}\right)\left(s(u) \rho e^{t_{0}}\right) \\
& =2 i \widetilde{\eta}_{k, h}^{j}\left(s(u) \rho e^{t_{0}}\right)=2 i f\left(t_{0}\right) .
\end{aligned}
$$

And this time, $f(0)=\tilde{\eta}_{k, h}^{j}(s(u) \rho)=\frac{1}{\rho} \eta_{k, h}^{j}(u)$, so that for $f$

$$
\left\{\begin{array}{l}
f^{\prime}=2 i f \\
f(0)=\frac{1}{\rho} \eta_{k, h}^{j}(u)
\end{array}\right.
$$

Its unique solution is $f(t)=\eta_{k, h}^{j}(u) \frac{e^{2 i t}}{\rho}$, which implies

$$
\widetilde{\eta}_{k, h}^{j}\left(s(u) \rho e^{i t}\right)=\eta_{k, h}^{j}(u) \frac{e^{2 i t}}{\rho}=\left(\frac{\pi^{*} \eta_{k, h}^{j}}{r^{3}}\right)\left(s(u) \rho e^{i t}\right) \rho^{2} e^{2 i t} .
$$

Let now $z: \pi^{-1}(U) \rightarrow \mathbb{C}^{*}$ be as in the statement, then in particular for all $w \in \pi^{-1}(u)$, we have $w=s(u) z(u)$. Then, $\widetilde{\eta}_{k, h}^{j}(w)=z^{2} \frac{\pi^{*} \eta_{k, h}^{j}}{r^{3}}(w)$. So finally we have

$$
\begin{aligned}
\tilde{\eta} & =\Re\left(\widetilde{\eta}_{k, h}^{j} \widetilde{\theta}^{k} \otimes{\overline{\tilde{\theta}_{j}}} \otimes \widetilde{\theta}^{h}\right)=\Re\left(z^{2} \frac{\pi^{*} \eta_{k, h}^{j}}{r^{3}}\left(r \pi^{*} \theta^{k} \otimes r \pi^{*} \overline{\theta_{j}} \otimes r \pi^{*} \theta^{h}\right)\right) \\
& =\Re\left(z^{2} \pi^{*} \eta_{k, h}^{j} \pi^{*} \theta^{k} \otimes \pi^{*} \overline{\theta_{j}} \otimes \pi^{*} \theta^{h}\right)=\Re\left(z^{2} \pi^{*} \eta\right) .
\end{aligned}
$$

Definition 6.4 Given a section $s: U \rightarrow S$ with $U$ open subset of $M$, we will call the corresponding tensor $\eta$ found in Proposition 6.3 the deviance tensor with respect to $s$.

We can give a more global formulation of Proposition 6.3 in the following terms

Proposition 6.5 Given a projective special Kähler manifold $(\pi: \tilde{M} \rightarrow M, \nabla)$, there exists a map $\gamma: \widetilde{M} \rightarrow \sharp_{2} S_{3,0} M \subset T_{1,0} M \otimes T^{0,1} M \otimes T_{1,0} M$ of bundles over $M$, such that $\gamma(u a)=a^{2} \gamma(u)$ and for every local section $s: U \rightarrow S \subset \widetilde{M}$, the deviance induced by $s$ is $\eta=\gamma \circ s$.

Let $L:=\widetilde{M} \times_{\mathbb{C}^{*}} \mathbb{C}$, then $\gamma$ can be identified with a homomorphism of complex vector bundles $\hat{\gamma}: L \otimes L \rightarrow \#_{2} S_{3,0} M$ such that $\gamma(u)=\widehat{\gamma}([u, 1] \otimes[u, 1])$. 
Proof Let $u \in \widetilde{M}$, then there exists an open neighbourhood $U \subseteq M$ of $u$ and local trivialisation $\left(\left.\pi\right|_{\pi^{-1}(U)}, z\right): \pi^{-1}(U) \rightarrow U \times \mathbb{C}^{*}$ induced by a section $s: U \rightarrow S$ so, for all $w \in \pi^{-1}(U)$ we have $w=s(\pi(w)) z(w)$. Let now $\eta: U \rightarrow \sharp_{2} S_{3,0} M$ be the deviance corresponding to $s$; we define $\gamma(u):=z(u)^{2} \eta(p)$ where $p=\pi(u)$. This definition is independent on the choice of $s$. In order to prove it take another $s^{\prime}: U^{\prime} \rightarrow S$ with $p \in U^{\prime}$ and the corresponding $z^{\prime}$ and $\eta^{\prime}$, then, on $U \cap U^{\prime}$, there is a map $c:=z \circ s^{\prime}: U \cap U^{\prime} \rightarrow \mathbb{C}$ whose image is in $S^{1}$, as both $s$ and $s^{\prime}$ are sections of $S$. By definition, $s^{\prime}=s \cdot c$. Since $\quad s z=s^{\prime} z^{\prime}, \quad z(u)=z\left(s^{\prime}(p) z^{\prime}(u)\right)=z\left(s^{\prime}(p)\right) z^{\prime}(u)=c(p) z^{\prime}(u), \quad$ so $\quad z=z^{\prime} \pi^{*} c$. Now, by construction $\mathfrak{R}\left(z^{\prime 2} \pi^{*} \eta^{\prime}\right)=\tilde{\eta}=\mathfrak{R}\left(z^{2} \pi^{*} \eta\right)=\mathfrak{R}\left(z^{\prime 2} \pi^{*} c^{2} \pi^{*} \eta^{\prime}\right)$, so $\eta^{\prime}=c^{2} \eta$. Thus, $z(u)^{2} \eta(p)=z^{\prime}(u)^{2} c(p)^{2} \eta(p)=z^{\prime}(u)^{2} \eta^{\prime}(p)$, and thus, $\gamma$ is well defined.

Moreover, $\gamma(u a)=z(u a)^{2} \eta(\pi(u a))=z(u)^{2} a^{2} \eta(p)=a^{2} \gamma(u)$.

We can define the homomorphism $L \otimes L \rightarrow \#_{2} S_{3,0} M$ locally: given a section $s: U \rightarrow S$, we map $[u, w] \otimes\left[u^{\prime}, w^{\prime}\right]$ to $z(u) z\left(u^{\prime}\right) w w^{\prime} \cdot \eta_{p}^{s}$ where $p=\pi(u)=\pi\left(u^{\prime}\right)$. This map does not depend on the choice of the section as one can see from the relations above, and it is also independent on the representatives chosen of these classes; for the first class for example $z(u a) w=z(u) a w$.

This map commutes with the projections on $M$ and it is $\mathbb{C}$-linear on the fibres, so it is a complex vector bundle map.

Definition 6.6 We call $\gamma: S \rightarrow{ }_{2} S_{3,0} M$ of Proposition 6.5 the intrinsic deviance of the projective special Kähler manifold.

Remark 6.7 Given a section $s: U \rightarrow S$ and the corresponding function $z \in \mathcal{C}^{\infty}\left(\pi^{-1}(U), \mathbb{C}^{*}\right)$ such that $s z=\operatorname{id}_{\pi^{-1}(U)}$, we can compute $d z=z\left(\frac{1}{r} d r+i d \vartheta\right)$, since locally $z=r e^{i \vartheta}$. Notice that $\vartheta$ is not globally defined on $\pi^{-1}(U)$, but $d \vartheta$ and $e^{i \vartheta}$ are. Moreover,

$$
\frac{1}{z} d z=\frac{1}{r} d r+i d \vartheta \in \Omega^{1}\left(\pi^{-1}(U), \mathbb{C}\right)
$$

is a principal connection form, in fact it is equivariant for the action of $\mathbb{C}^{*}$ as $z(u a)=a z(u)$ for all $a \in \mathbb{C}$ and, given a complex number $a$ and its corresponding fundamental vector field $a^{\circ} \in \mathfrak{X}(\tilde{M})$,

$$
\frac{1}{z} d z\left(a^{\circ}\right)_{u}=\frac{1}{z} d z\left(\left.\frac{d}{d t} u e^{a t}\right|_{t=0}\right)=\left.\frac{1}{z(u)} \frac{d}{d t} z\left(u e^{a t}\right)\right|_{t=0}=\left.\frac{1}{z(u)} \frac{d}{d t} z(u) e^{a t}\right|_{t=0}=a .
$$

Remark 6.8 A local section $s: U \rightarrow S$ induces $\tau:=s^{*} \widetilde{\varphi}=s^{*} \varphi \in \Omega^{1}(U)$ such that on $\pi^{-1}(U)$

$$
\widetilde{\varphi}=d \vartheta+\pi^{*} \tau,
$$

and thus, on $\pi_{S}^{-1}(U)$ :

$$
\varphi=\left.d \vartheta\right|_{S}+\pi_{S}^{*} \tau
$$

If we consider in fact the form $\widetilde{\varphi}-d \vartheta$, we notice that it is basic, as it can also be seen as the difference of two connection forms on $\pi^{-1}(U)$ (namely (3) and (8)) up to a multiplication by $i$. Therefore, $\widetilde{\varphi}-d \vartheta=\pi^{*} \tau$ for some $\tau \in \Omega^{1}(U)$. The second equation is simply obtained from the first by restriction to $S \subseteq \widetilde{M}$. 


\section{Characterisation theorem}

In this section, we prove our main theorem, characterising projective special Kähler manifolds in terms of the deviance. We start by deriving necessary conditions on the deviance, reflecting the curvature conditions of Proposition 3.2.

Proposition 7.1 For a projective special Kähler manifold $(\pi: \widetilde{M} \rightarrow M, \nabla)$ with $(\widetilde{M}, \widetilde{g}, \widetilde{I}, \widetilde{\omega}, \nabla, \xi)$, and a local section $s: U \rightarrow S$, then the corresponding deviance $\eta$ satisfies

$$
d^{L C} \eta=2 i \tau \wedge \eta
$$

where $\tau=s^{*} \varphi \in \Omega^{1}(U)$.

Proof Thanks to Proposition 6.3, we know that there exists $z=r e^{i \vartheta}$ and $\eta \in T_{1,0} U \otimes T^{0,1} U \otimes T_{1,0} U$ such that on $\pi^{-1}(U)$ we have $\tilde{\eta}=\mathfrak{R}\left(z^{2} \pi^{*} \eta\right)$.

Now we would like to describe $\tilde{d}^{L C} \tilde{\eta}$ in terms of $d^{L C} \eta$. Notice that

$$
\begin{aligned}
\tilde{d}^{L C} \widetilde{\eta} & =\widetilde{d}^{L C} \mathfrak{R}\left(z^{2} \pi^{*} \eta\right)=\mathfrak{R}\left(\tilde{d}^{L C}\left(z^{2} \pi^{*} \eta\right)\right)=\mathfrak{R}\left(2 z d z \wedge \pi^{*} \eta+z^{2} \widetilde{d}^{L C} \pi^{*} \eta\right) \\
& =\mathfrak{R}\left(z^{2}\left(2\left(\frac{1}{r} d r+i d \vartheta\right) \wedge \pi^{*} \eta+\widetilde{d}^{L C} \pi^{*} \eta\right)\right) .
\end{aligned}
$$

The next step is to compute $\widetilde{d}^{L C} \pi^{*} \eta$, but since we are using the Levi-Civita connection, it is equivalent to compute $\sharp_{2}\left(\tilde{d}^{L C} \pi^{*} \sigma\right)$, where $\sigma=b_{2} \eta \in S_{3,0} U$. Let us consider a local coframe $\theta$ in $M$ and the corresponding lifting $\widetilde{\theta}$ as in (4), so that we can denote explicitly $\sigma=\sigma_{k, j, h} \theta^{k} \otimes \theta^{j} \otimes \theta^{h}$. We have

$$
\begin{aligned}
\widetilde{\nabla}^{L C} \pi^{*} \theta^{k} & =\widetilde{\nabla}^{L C} \frac{\widetilde{\theta}^{k}}{r}=-\frac{d r}{r^{2}} \otimes \widetilde{\theta}^{k}-\frac{1}{r}\left(\left(\widetilde{\omega}^{L C}\right)_{j}^{k} \otimes \widetilde{\theta}^{j}\right) \\
& =-\frac{d r}{r} \otimes \pi^{*} \theta^{k}-\frac{1}{r}\left(\sum_{j=1}^{n} \pi^{*}\left(\omega^{L C}\right)_{j}^{k} \otimes \widetilde{\theta}^{j}+i \widetilde{\varphi} \otimes \widetilde{\theta}^{j}+\pi^{*} \theta^{k} \otimes \theta^{n+1}\right) \\
& =-\frac{d r}{r} \otimes \pi^{*} \theta^{k}-\pi^{*}\left(\left(\omega^{L C}\right)_{j}^{k} \otimes \theta^{j}\right)-i \widetilde{\varphi} \otimes \pi^{*} \theta^{j}-\pi^{*} \theta^{k} \otimes \frac{1}{r} \theta^{n+1} \\
& =\pi^{*}\left(\nabla^{L C} \theta^{k}\right)-\frac{1}{r} \theta^{n+1} \otimes \pi^{*} \theta^{k}-\pi^{*} \theta^{k} \otimes \frac{1}{r} \theta^{n+1} .
\end{aligned}
$$

We can now compute the following for $X \in \mathfrak{X}\left(\pi^{-1}(U)\right)$ : 


$$
\begin{aligned}
\widetilde{\nabla}_{X}^{L C} \pi^{*} \sigma & =\widetilde{\nabla}_{X}^{L C} \pi^{*}\left(\sigma_{k, j, h} \theta^{k} \otimes \theta^{j} \otimes \theta^{h}\right)=\widetilde{\nabla}_{X}^{L C}\left(\pi^{*} \sigma_{k, j, h} \pi^{*} \theta^{k} \otimes \pi^{*} \theta^{j} \otimes \pi^{*} \theta^{h}\right) \\
= & d \pi^{*} \sigma_{k, j, h}(X) \theta^{k} \otimes \theta^{j} \otimes \theta^{h}+\pi^{*} \sigma_{k, j, h}\left(\widetilde{\nabla}_{X}^{L C} \pi^{*} \theta^{k} \otimes \pi^{*} \theta^{j} \otimes \pi^{*} \theta^{h}\right. \\
& \left.+\pi^{*} \theta^{k} \otimes \widetilde{\nabla}_{X}^{L C} \pi^{*} \theta^{j} \otimes \pi^{*} \theta^{h}+\pi^{*} \theta^{k} \otimes \pi^{*} \theta^{j} \otimes \widetilde{\nabla}_{X}^{L C} \pi^{*} \theta^{h}\right) \\
= & \pi^{*} d \sigma_{k, j, h}(X) \theta^{k} \otimes \theta^{j} \otimes \theta^{h}+\pi^{*} \sigma_{k, j, h} \pi^{*}\left(\nabla^{L C} \theta^{k}\right)_{X} \otimes \pi^{*} \theta^{j} \otimes \pi^{*} \theta^{h} \\
& +\pi^{*} \sigma_{k, j, h} \pi^{*} \theta^{k} \otimes \pi^{*}\left(\nabla^{L C} \theta^{j}\right)_{X} \otimes \pi^{*} \theta^{h} \\
& +\pi^{*} \sigma_{k, j, h} \pi^{*} \theta^{k} \otimes \pi^{*} \theta^{j} \otimes \pi^{*}\left(\nabla^{L C} \theta^{j}\right)_{X}-\frac{3}{r} \widetilde{\theta}^{n+1}(X) \pi^{*} \sigma \\
& -\frac{1}{r}\left(\pi^{*} \sigma_{k, j, h} \pi^{*} \theta^{k}(X) \widetilde{\theta}^{n+1} \otimes \pi^{*} \theta^{j} \otimes \pi^{*} \theta^{h}\right. \\
& +\pi^{*} \sigma_{k, j, h} \pi^{*} \theta^{k} \otimes \pi^{*} \theta^{j}(X) \widetilde{\theta}^{n+1} \otimes \pi^{*} \theta^{h} \\
& \left.+\pi^{*} \sigma_{k, j, h} \pi^{*} \theta^{k} \otimes \pi^{*} \theta^{j} \otimes \pi^{*} \theta^{h}(X) \widetilde{\theta}^{n+1}\right) \\
= & \pi^{*}\left(\nabla^{L C} \sigma\right)_{X}-\frac{2}{r} \widetilde{\theta}^{n+1}(X) \pi^{*} \sigma-\frac{1}{r} \widetilde{\theta}^{n+1}(X) \pi^{*} \sigma-\frac{1}{r} \widetilde{\theta}^{n+1} \otimes \pi^{*} \sigma(X, \cdot, \cdot) \\
& -\frac{1}{r} \pi^{*} \sigma\left(\cdot, X \otimes \widetilde{\theta}^{n+1}, \cdot\right)-\frac{1}{r} \pi^{*} \sigma\left(\cdot, \cdot, X \otimes \widetilde{\theta}^{n+1}\right) .
\end{aligned}
$$

In general then, if $\sigma=\theta^{k} \otimes \sigma_{k}$, where $\sigma_{k}=\sigma_{k, j, h} \theta^{j} \otimes \theta^{h} \in S_{2,0} U$, we have by symmetry

$$
\begin{aligned}
\widetilde{\nabla}^{L C} \pi^{*} \sigma= & \pi^{*}\left(\nabla^{L C} \sigma\right)-\frac{2}{r} \widetilde{\theta}^{n+1} \otimes \pi^{*} \sigma-\frac{2}{r}\left(\left(\widetilde{\theta}^{n+1}\right)\left(\pi^{*} \theta^{k}\right)\right) \otimes \pi^{*}\left(\sigma_{k, j, h} \theta^{j} \otimes \theta^{h}\right) \\
& -\frac{2}{r}\left(\pi^{*}\left(\sigma_{k, j, h} \theta^{k} \otimes \theta^{j}\right) \otimes\left(\left(\widetilde{\theta}^{n+1}\right)\left(\pi^{*} \theta^{h}\right)\right)\right) .
\end{aligned}
$$

Notice in particular that the last two rows are symmetric in the first two indices.

In order to compute $\widetilde{d}^{L C} \pi^{*} \sigma$ we need to antisymmetrise $\widetilde{\nabla}^{L C} \pi^{*} \sigma$ in the first two indices and multiply by two, so only the first row survives and we get

$$
\tilde{d}^{L C} \pi^{*} \sigma=\pi^{*}\left(d^{L C} \sigma\right)-\frac{2}{r} \widetilde{\theta}^{n+1} \wedge \pi^{*} \sigma
$$

and therefore

$$
\widetilde{d}^{L C} \pi^{*} \eta=\pi^{*}\left(d^{L C} \eta\right)-\frac{2}{r} \widetilde{\theta}^{n+1} \wedge \pi^{*} \eta
$$

Substituting this value in (9), we obtain

$$
\begin{aligned}
\widetilde{d}^{L C} \widetilde{\eta} & =\mathfrak{R}\left(z^{2}\left(2\left(\frac{1}{r} d r+i d \vartheta\right) \wedge \pi^{*} \eta+\pi^{*}\left(d^{L C} \eta\right)-\frac{2}{r} \widetilde{\theta}^{n+1} \wedge \pi^{*} \eta\right)\right) \\
& =\mathfrak{R}\left(z^{2}\left(\pi^{*} d^{L C} \eta-2 i(\widetilde{\varphi}-d \vartheta) \wedge \pi^{*} \eta\right)\right) .
\end{aligned}
$$

As observed in Remark 6.8, $\widetilde{\varphi}-d \vartheta=\pi^{*} \tau$, so we have

$$
\widetilde{d}^{L C} \widetilde{\eta}=\Re\left(z^{2} \pi^{*}\left(d^{L C} \eta-2 i \tau \wedge \eta\right)\right) .
$$

From Proposition 3.2, we know that $\tilde{d}^{L C} \tilde{\eta}=0$, and since $\eta \in \Omega^{1}\left(U, T_{0,1} \otimes T^{1,0}\right), \eta$ and $\bar{\eta}$ are linearly independent, so this quantity vanishes if and only if $z^{2} \pi^{*}\left(d^{L C} \eta-2 i \tau \wedge \eta\right)$ does. Therefore, 


$$
d^{L C} \eta-2 i \tau \wedge \eta=0
$$

ending the proof.

Let us now look at the final ingredient of the curvature tensor, that is $\frac{1}{2}[\tilde{\eta} \wedge \tilde{\eta}]$. In the setting of Proposition 6.3, given a section $s: U \rightarrow S$, and the induced deviance $\eta$, then

$$
\begin{aligned}
\frac{1}{2}[\widetilde{\eta} \wedge \widetilde{\eta}] & =\frac{1}{2}\left[\Re\left(z^{2} \pi^{*} \eta\right) \wedge \mathfrak{R}\left(z^{2} \pi^{*} \eta\right)\right]=\frac{1}{2}\left[z^{2} \pi^{*} \eta+\bar{z}^{2} \pi^{*} \bar{\eta} \wedge z^{2} \pi^{*} \eta+\bar{z}^{2} \pi^{*} \bar{\eta}\right] \\
& =\frac{1}{2} \mathfrak{R}\left(z^{4}\left[\pi^{*} \eta \wedge \pi^{*} \eta\right]\right)+|z|^{4}\left[\pi^{*} \eta \wedge \pi^{*} \bar{\eta}\right] .
\end{aligned}
$$

We can compute this tensor for a local coframe $\theta$ on $M$. Since we have

$$
\pi^{*} \theta^{k} \circ \pi^{*} \theta_{h}=\frac{1}{r} \widetilde{\theta}^{k}\left(\frac{1}{r} \widetilde{\theta}_{h}\right)=\frac{1}{r^{2}} \widetilde{\theta}^{k}\left(\widetilde{\theta}_{h}\right)=\frac{1}{r^{2}} \delta_{h}^{k}=\frac{1}{r^{2}} \pi^{*}\left(\theta^{k} \circ \theta_{h}\right)
$$

and $\pi^{*} \theta^{k} \circ \pi^{*} \overline{\theta_{h}}=\pi^{*} \bar{\theta}^{k} \circ \pi^{*} \theta_{h}=0$, then

$$
\begin{aligned}
{\left[\pi^{*} \eta \wedge \pi^{*} \eta\right] } & =\left[\pi^{*} \eta_{k, h}^{j} \pi^{*} \theta^{k} \otimes \pi^{*} \overline{\theta_{j}} \otimes \pi^{*} \theta^{h} \wedge \pi^{*} \eta_{k^{\prime}, h^{\prime}}^{j^{\prime}} \pi^{*} \theta^{k^{\prime}} \otimes \pi^{*} \overline{\theta_{j^{\prime}}} \otimes \pi^{*} \theta^{h^{\prime}}\right] \\
& =\pi^{*} \eta_{k, h}^{j} \pi^{*} \theta^{k} \wedge \pi^{*} \eta_{k^{\prime}, h^{\prime}}^{j^{\prime}} \pi^{*} \theta^{k^{\prime}} \otimes\left[\pi^{*} \overline{\theta_{j}} \otimes \pi^{*} \theta^{h}, \pi^{*} \overline{\theta_{j^{\prime}}} \otimes \pi^{*} \theta^{h^{\prime}}\right]=0
\end{aligned}
$$

and

$$
\begin{aligned}
{\left[\pi^{*} \eta \wedge \pi^{*} \bar{\eta}\right] } & =\left[\pi^{*} \eta_{k, h}^{j} \pi^{*} \theta^{k} \otimes \pi^{*} \overline{\theta_{j}} \otimes \pi^{*} \theta^{h} \wedge \pi^{*} \overline{\eta_{k^{\prime}, h^{\prime}}^{j^{\prime}}} \pi^{*} \overline{\theta^{k^{\prime}}} \otimes \pi^{*} \theta_{j^{\prime}} \otimes \pi^{*} \overline{\theta^{h^{\prime}}}\right] \\
& =\pi^{*} \eta_{k, h}^{j} \pi^{*} \theta^{k} \wedge \pi^{*} \overline{\eta_{k^{\prime}, h^{\prime}}^{\prime}} \pi^{*} \overline{\theta^{k^{\prime}}} \otimes\left[\pi^{*} \overline{\theta_{j}} \otimes \pi^{*} \theta^{h}, \pi^{*} \theta_{j^{\prime}} \otimes \pi^{*} \overline{\theta^{h^{\prime}}}\right] \\
& \left.=\pi^{*}\left(\eta_{k, h}^{j} \theta^{k} \wedge \overline{\eta_{k^{\prime}, h^{\prime}}^{j^{\prime}}} \overline{{k^{k^{\prime}}}^{\prime}}\right) \otimes \frac{1}{r^{2}} \pi^{*}\left(\overline{\theta_{j}} \otimes \theta^{h}\left(\theta_{j^{\prime}}\right) \otimes \overline{\theta^{h^{\prime}}}-\theta_{j^{\prime}} \otimes \overline{\theta^{h^{\prime}}} \overline{\left(\theta_{j}\right.}\right) \otimes \theta^{h}\right) \\
& =\frac{1}{r^{2}} \pi^{*}[\eta \wedge \bar{\eta}] .
\end{aligned}
$$

Therefore

$$
\frac{1}{2}[\tilde{\eta} \wedge \tilde{\eta}]=\frac{|z|^{4}}{r^{2}} \pi^{*}[\eta \wedge \bar{\eta}]=r^{2} \pi^{*}[\eta \wedge \bar{\eta}]
$$

Remark 7.2 Note that $[\eta \wedge \bar{\eta}]$ is independent on the local coframe, and if we consider another section such that $s^{\prime}=s a$ on the intersection of their domains, with $a$ taking values in $S^{1}$, if $\eta^{\prime}$ is the deviance corresponding to $s^{\prime}$, then $\left[\eta^{\prime} \wedge \overline{\eta^{\prime}}\right]=[\eta a \wedge \overline{\eta a}]=|a|^{2}[\eta \wedge \bar{\eta}]=[\eta \wedge \bar{\eta}]$. So, there is a globally defined section $M \rightarrow S^{2}(\mathfrak{u}(n))$ mapping $p$ to $\left[\eta_{p} \wedge \overline{\eta_{p}}\right]$.

For a projective special Kähler manifold $(\pi: \widetilde{M} \rightarrow M, \nabla)$ of real dimension $2 n$, Proposition 3.2, interpreted in the light of the last observations and the ones made in Section 5 (see Remark 5.4), says that $0=r^{2} \pi^{*}\left(\Omega^{L C}+\Omega_{\mathbb{P}_{C}^{n}}+[\eta \wedge \bar{\eta}]\right)$; thus, we have the following equation:

$$
\Omega^{L C}+\Omega_{\mathbb{P}_{\mathbb{C}}^{n}}+[\eta \wedge \bar{\eta}]=0
$$

This is a curvature tensor, so we can compute its Ricci and scalar component. 
Proposition 7.3 Let $(\pi: \tilde{M} \rightarrow M, \nabla)$ be a projective special Kähler manifold of dimension $2 n$, then

$$
\begin{gathered}
\operatorname{Ric}_{M}(X, Y)+2(n+1) g(X, Y)-\mathfrak{R}\left(h\left(\overline{\eta_{X}}, \eta_{Y}\right)\right)=0 ; \\
\operatorname{scal}_{M}+2(n+1)-\frac{2}{n}\|\eta\|_{h}^{2}=0 .
\end{gathered}
$$

Proof The first summand in (11) gives the Ricci tensor of $M$, the second gives the Ricci tensor of the projective space (6). In order to compute the last term, consider a unitary frame $\theta$; from previous computations,

$$
\begin{aligned}
{[\eta \wedge \bar{\eta}] } & =\left(\eta_{k, h}^{j} \theta^{k} \wedge \overline{\eta_{k^{\prime}, h^{\prime}}} \overline{\theta^{k^{\prime}}}\right) \otimes\left(\delta_{j^{\prime}}^{h} \overline{\theta_{j}} \otimes \overline{\theta^{h^{\prime}}}-\delta_{j}^{h^{\prime}} \theta_{j^{\prime}} \otimes \theta^{h}\right) \\
& =\Re\left(\eta_{k, h}^{j} \overline{\eta_{k^{\prime}, h^{\prime}}^{h}} \theta^{k} \wedge \overline{\theta^{k^{\prime}}} \otimes \overline{\theta_{j}} \otimes \overline{\theta^{h^{\prime}}}\right)
\end{aligned}
$$

then the Ricci component $\operatorname{Ric}([\eta \wedge \bar{\eta}])$ evaluated on $X=\Re\left(X^{k} \theta_{k}\right)$ and $Y=\Re\left(Y^{k} \theta_{k}\right)$ is the trace of $[\eta \wedge \bar{\eta}](\cdot, Y) X$, which is

$$
\begin{aligned}
{[\eta \wedge \bar{\eta}](\cdot, Y) X } & =\eta_{k, h}^{j} \overline{\eta_{u, v}^{h}}\left(\theta^{k} \overline{Y^{u}}-Y^{k} \overline{\theta^{u}}\right) \otimes \overline{\theta_{j}} \otimes \overline{X^{v}}+\overline{\eta_{k, h}^{j}} \eta_{u, v}^{h}\left(\overline{\theta^{k}} Y^{u}-\overline{Y^{u}} \theta^{k}\right) \otimes \theta_{j} \otimes X^{v} \\
& =\Re\left(\eta_{k, h}^{j} \overline{\eta_{u, v}^{h}}\left(\theta^{k} \overline{Y^{u}}-Y^{k} \overline{\theta^{u}}\right) \otimes \overline{\theta_{j}} \otimes \overline{X^{v}}\right) .
\end{aligned}
$$

Its trace is therefore

$$
-\Re\left(\eta_{k, h}^{j} \overline{\eta_{j, v}^{h}} Y^{k} \overline{X^{v}}\right)=-\mathfrak{R}\left(\eta_{k, h}^{j} \overline{\eta_{u, j}^{h}} Y^{k} \overline{X^{u}}\right)=-\Re\left(h\left(\overline{\eta_{X}}, \eta_{Y}\right)\right)
$$

or equivalently, $\operatorname{Ric}([\eta \wedge \bar{\eta}])=-\Re\left(\overline{\eta_{u, j}^{h}} \eta_{k, h}^{j} \overline{\theta^{u}} \theta^{k}\right)$. Thus, we obtain (12).

From this tensor, we can now obtain (13) by computing the scalar component, that is by taking the trace, raising the indices with $g$ and then dividing it by the dimension of $M$. Thus, the first summand gives $\operatorname{scal}_{M}$, the second gives $2(n+1)$ and the third

$$
\begin{aligned}
\frac{1}{2 n} \operatorname{tr}\left(-\Re\left(\overline{\eta_{u, j}^{h}} \eta_{k, h}^{j}\left(\overline{\theta^{u}}\right)_{\sharp} \theta^{k}\right)\right) & =-\frac{1}{2 n} \operatorname{tr}\left(\Re\left(\overline{\eta_{u, j}^{h}} \eta_{k, h}^{j}\left(2 \theta_{u}\right) \theta^{k}\right)\right) \\
& =-\frac{1}{n} \sum_{j, h, k} \mathfrak{R}\left(\eta_{k, h}^{j} \overline{\eta_{k, j}^{h}}\right)=-\frac{2}{n}\|\eta\|_{h}^{2} .
\end{aligned}
$$

In particular, since the norm of $\eta$ is non-negative, we obtain a lower bound for the scalar curvature:

Corollary 7.4 Let $(\pi: \widetilde{M} \rightarrow M, \nabla)$ be a projective special Kähler manifold, then

$$
\operatorname{scal}_{M} \geq-2(n+1) \text {. }
$$

Equality holds at a point if and only if the deviance vanishes at that point.

Remark 7.5 The lower bound is reached by projective special Kähler manifolds with zero deviance; we will see that this condition characterises the complex hyperbolic space (Proposition 9.5). 
We can now state the main result:

Theorem 7.6 On a 2n-dimensional Kähler manifold $(M, g, I, \omega)$, to give a projective special Kähler structure is equivalent to give an $S^{1}$-bundle $\pi_{S}: S \rightarrow M$ endowed with a connection form $\varphi$ and a bundle map $\gamma: S \rightarrow \sharp_{2} S_{3,0} M$ such that:

1. $d \varphi=-2 \pi_{S}^{*} \omega$

2. $\gamma(u a)=a^{2} \gamma(u)$ for all $a \in S^{1}$;

3. for a certain choice of an open covering $\left\{U_{\alpha} \mid \alpha \in \mathcal{A}\right\}$ of M and a family $\left\{s_{\alpha}: U_{\alpha} \rightarrow S\right\}_{\alpha \in \mathcal{A}}$ of sections, denoting by $\eta_{\alpha}$ the local 1-form taking values in $T^{0,1} M \otimes T_{1,0} M$ determined by $\gamma \circ \mathrm{s}_{\alpha}$, for all $\alpha \in \mathcal{A}$ :

D1 $\Omega^{L C}+\Omega_{\mathbb{P}_{\mathrm{C}}^{n}}+\left[\eta_{\alpha} \wedge \overline{\eta_{\alpha}}\right]=0$

D2 $d^{L C} \eta_{\alpha}=2 i s_{\alpha}^{*} \varphi \wedge \eta_{\alpha}$.

In this case, 3 is satisfied by every such family of sections.

Proof Given a projective special Kähler manifold, we define $S:=r^{-1}(1) \subset \widetilde{M}$ and $\varphi:=-\left.l_{\xi} \omega\right|_{S}$. The principal action on $S$ is generated by $I \xi$ which is tangent to $S$ since $T_{u} S=\operatorname{ker}(d r)$ and $d r(I \xi)=-\frac{1}{r} \xi^{b}(I \xi)=-\frac{\widetilde{g}(\xi, I \xi)}{r}$. The curvature is then $d \varphi=-2 \pi_{S}^{*} \omega$ as shown in Remark 4.4, so the first point is satisfied. The second condition holds thanks to Proposition 6.5. For the third point, we get D1 from the arguments leading to equation (11) and D2 from Proposition 7.1.

In order to prove the other direction, define $\widetilde{M}:=S \times \mathbb{R}^{+}, \pi:=\pi_{S} \circ \pi_{1}: \widetilde{M} \rightarrow M$, and $t:=\pi_{2} \in \mathcal{C}^{\infty}\left(\tilde{M}, \mathbb{R}^{+}\right)$, where $\pi_{1}: S \times \mathbb{R}^{+} \rightarrow S$ and $\pi_{2}: S \times \mathbb{R}^{+} \rightarrow \mathbb{R}^{+}$are the projections. Let $\widetilde{\varphi}:=\pi_{1}^{*} \varphi$, in particular $d \widetilde{\varphi}=\pi_{1}^{*} d \varphi=-2 \pi^{*} \omega$ as expected. Define now

$$
\widetilde{g}:=t^{2} \pi^{*} g-t^{2} \widetilde{\varphi}^{2}-d t^{2}
$$

which is non-degenerate, since $r \tilde{\varphi}$ and $d t$ are linearly independent and transverse to $\pi$, so we can form a basis for the 1 -forms according to which we can see that $\widetilde{g}$ has signature $(2 n, 2)$. Extend now $I$ to $\widetilde{I}$ so that $\widetilde{I} \cdot\left(\pi^{*} \alpha\right)=\pi^{*} I \alpha$ for all $\alpha \in T^{*} M$ and $\widetilde{I} \cdot(d t)=t \widetilde{\varphi}$.

The metric $\widetilde{g}$ is compatible with $\widetilde{I}$ since

$$
\left.\widetilde{I} \cdot \widetilde{g}=t^{2} \widetilde{I} \cdot \pi^{*} g-(\widetilde{I} \cdot t \widetilde{\varphi})^{2}-\widetilde{I} \cdot d t\right)^{2}=t^{2} \pi^{*}(I \cdot g)-(-d t)^{2}-(t \widetilde{\varphi})^{2}=t^{2} \pi^{*}(I \cdot g)-d t^{2}-t^{2} \widetilde{\varphi}^{2}=\widetilde{g} .
$$

We thus have a Kähler manifold $(\widetilde{M}, \widetilde{g}, \widetilde{I}, \widetilde{\omega})$, where

$$
\widetilde{\omega}:=t^{2} \pi^{*} \omega+t \tilde{\varphi} \wedge d t
$$

Let $\xi:=t \partial_{t}$ where $\partial_{t}$ is the vector field corresponding to the coordinate derivation on $\mathbb{R}^{+}$. Notice that the function $r=\sqrt{-\widetilde{g}(\xi, \xi)}$ coincides with $t$, as $\sqrt{-\widetilde{g}\left(t \partial_{t}, t \partial_{t}\right)}=\sqrt{-t^{2} \widetilde{g}\left(\partial_{t}, \partial_{t}\right)}=t$. In particular, $\widetilde{g}(\xi, \xi)=-t^{2} \neq 0 \quad$ and $\widetilde{g}(\widetilde{I} \xi, \widetilde{I} \xi)=\widetilde{g}(\xi, \xi)<0$, so $\widetilde{g}$ is negative definite on $\langle\xi, I \xi\rangle$ and hence positive definite on the orthogonal complement.

Let now $\theta$ be a unitary coframe on an open subset $U \subseteq M$, then we can lift it to a complex coframe $\widetilde{\theta}$ on $\pi^{-1}(U)$ defined as in (4). It is straightforward to check that $\widetilde{\theta}$ is adapted to the pseudo-Kähler structure of $\widetilde{M}$. Notice that the proof of Proposition 5.2 is still valid in this situation even though we do not know whether $\widetilde{M} \rightarrow M$ has a structure of projective 
special Kähler manifold; this gives us a description of the Levi-Civita connection form on $\widetilde{M}$ with respect to $\widetilde{\theta}$. Notice that $\widetilde{\theta}^{k}(\xi)=0$ for $k \leq n$ and $\widetilde{\theta}^{n+1}(\xi)=d t\left(t \partial_{t}\right)+i \widetilde{\varphi}\left(t \partial_{t}\right)=t$ so $\xi=\Re\left(\widetilde{\theta}_{n+1}\right)$. We can thus compute

$$
\begin{aligned}
\widetilde{\nabla}^{L C} \xi & =d t \otimes \Re\left(\widetilde{\theta}_{n+1}\right)+t \widetilde{\nabla}^{L C} \mathfrak{R}\left(\tilde{\theta}_{n+1}\right) \\
& =\Re\left(d t \otimes \widetilde{\theta}_{n+1}\right)+\frac{t}{r} \Re\left(\sum_{k=1}^{n} \widetilde{\theta}^{k} \otimes \widetilde{\theta}_{k}+i \operatorname{Im}\left(\widetilde{\theta}^{n+1}\right) \otimes \widetilde{\theta}_{n+1}\right) \\
& =\Re\left(\sum_{k=1}^{n+1} \widetilde{\theta}^{k} \otimes \widetilde{\theta}_{k}\right)=\mathrm{id} .
\end{aligned}
$$

Each section $s_{\alpha}$ corresponds to the trivialisation $\left(\left.\pi\right|_{\pi^{-1}(U)}, z_{\alpha}\right): \pi^{-1} U \rightarrow U \times \mathbb{C}^{*}$ in the sense that $s(\pi(u)) \cdot z_{\alpha}(u)=u$ for all $u \in \pi^{-1}\left(U_{\alpha}\right)$. For all $\alpha$ on $\pi^{-1}\left(U_{\alpha}\right)$, define the tensor $\tilde{\eta}_{\alpha}:=\Re\left(z_{\alpha}^{2} \pi^{*} \eta_{\alpha}\right)$. The family $\left\{\tilde{\eta}_{\alpha}\right\}_{\alpha \in \mathcal{A}}$ is compatible on intersections $U_{1} \cap U_{2}$, in fact if $s_{1}=c s_{2}$ for $c \in \mathrm{U}(1)$, then $z_{2}=c z_{1}$ and $\eta_{1}=\gamma \circ s_{1}=\gamma \circ c s_{2}=c^{2} \gamma \circ s_{2}=c^{2} \eta_{2}$, so

$$
\tilde{\eta_{1}}=\Re\left(z_{1}^{2} \pi^{*} \eta_{1}\right)=\mathfrak{R}\left(z_{1}^{2} c^{2} \pi^{*} \eta_{2}\right)=\mathfrak{R}\left(z_{2}^{2} \pi^{*} \eta_{2}\right)=\tilde{\eta_{2}} .
$$

Therefore, this family glues to form a tensor $\tilde{\eta} \in \sharp_{2} S^{3} \tilde{M}$.

We can build another connection $\nabla:=\widetilde{\nabla}^{L C}+\tilde{\eta}$. Notice that $\nabla \xi=\widetilde{\nabla}^{L C} \xi+\widetilde{\eta}(\xi)=\mathrm{id}+\Re\left(z_{\alpha}^{2} \pi^{*} \eta_{\alpha}\right)(\xi)=$ id because locally $\eta_{\alpha}$ is horizontal for all $\alpha$.

In order to prove that $\nabla$ is symplectic, since the Levi-Civita connection is symplectic, it is enough to prove that $\widetilde{\omega}(\widetilde{\eta}, \cdot)+\widetilde{\omega}(\cdot, \widetilde{\eta})=0$. Locally, $\widetilde{\omega}=\frac{1}{2 i} \sum_{k=1}^{n+1} \overline{\widetilde{\theta}^{k}} \wedge \widetilde{\theta}^{k}$ and in fact, for all $X=\mathfrak{R}\left(X^{k} \widetilde{\theta}_{k}\right), Y=\mathfrak{R}\left(Y^{k} \widetilde{\theta}_{k}\right), Z=\mathfrak{R}\left(Z^{k} \widetilde{\theta}_{k}\right)$ vector fields on $\widetilde{M}$ :

$$
\begin{aligned}
2 i\left(\widetilde{\omega}\left(\widetilde{\eta}_{X} Y, Z\right)+\widetilde{\omega}\left(Y, \widetilde{\eta}_{X} Z\right)\right)= & \sum_{k=1}^{n+1}\left(\overline{\widetilde{\theta}^{k}}\left(\widetilde{\eta}_{X} Y\right) \widetilde{\theta}^{k}(Z)-\widetilde{\theta}^{k}\left(\widetilde{\eta}_{X} Y\right) \overline{\widetilde{\theta}^{k}}(Z)\right. \\
& \left.+\overline{\widetilde{\theta}^{k}}(Y) \wedge \widetilde{\theta}^{k}\left(\widetilde{\eta}_{X} Z\right)-\widetilde{\theta}^{k}(Y) \wedge \overline{\widetilde{\theta}^{k}}\left(\widetilde{\eta}_{X} Z\right)\right) \\
= & \sum_{k=1}^{n+1}\left(z \pi^{*} \eta_{u, v}^{k} X^{u} Y^{v} Z^{k}-\overline{Z^{k}} \bar{z}^{2} \overline{\pi^{*} \eta_{u, v}^{k}} \overline{X^{u} Y^{v}}\right. \\
& \left.+\bar{Y}^{k} \bar{z}^{2} \overline{\pi^{*} \eta_{u, v}^{k}} \overline{X^{u} Z^{v}}-z^{2} \pi^{*} \eta_{u, v}^{k} X^{u} Z^{v} Y^{k}\right) \\
= & \sum_{k=1}^{n+1} \Re\left(z^{2} \pi^{*} \eta_{u, v}^{k} X^{u} Y^{v} Z^{k}-z^{2} \pi^{*} \eta_{u, v}^{k} X^{u} Z^{v} Y^{k}\right) \\
= & \sum_{k=1}^{n+1} \Re\left(z^{2} \pi^{*}\left(\eta_{u, v}^{k}-\eta_{u, k}^{v}\right) X^{u} Y^{v} Z^{k}\right) .
\end{aligned}
$$

By the symmetry of $\eta$, this quantity vanishes.

Proving that $d^{\nabla} \widetilde{I}=0$, is equivalent to proving that $\nabla \widetilde{I}$ is symmetric in the two covariant indices, and thus, $\nabla \widetilde{I}=\widetilde{\nabla}^{L C} \widetilde{I}+[\widetilde{\eta}, \widetilde{I}]=[\widetilde{\eta}, \widetilde{I}]$. Since $\widetilde{I}=\Re\left(i \widetilde{\theta}_{k} \widetilde{\theta}^{k}\right)$, we have 


$$
\begin{aligned}
& {[\tilde{\eta}, \widetilde{I}]=i z^{2} \pi^{*} \eta_{v, w}^{u} \widetilde{\theta}^{v} \otimes \overline{\widetilde{\theta}_{u}} \otimes \widetilde{\theta}^{w}-i \overline{i z^{2} \pi^{*} \eta_{v, w}^{u} \widetilde{\theta}^{v} \otimes \overline{\widetilde{\theta}}_{u} \otimes \widetilde{\theta}^{w}}} \\
& +i z^{2} \pi^{*} \eta_{v, w}^{u} \widetilde{\theta}^{v} \otimes \overline{\widetilde{\theta}}_{u} \otimes \widetilde{\theta}^{w}-i \overline{z^{2} \pi^{*} \eta_{v, w}^{u} \widetilde{\theta}^{v} \otimes \overline{\widetilde{\theta}_{u}} \otimes \widetilde{\theta}^{w}}=2 i \widetilde{\eta}=-2 \widetilde{I} \widetilde{\eta},
\end{aligned}
$$

which is symmetric, proving $d^{\nabla} I=0$.

For the flatness of $\nabla$, we compute the curvature locally

$$
\Omega^{\nabla}=d \omega^{\nabla}+\frac{1}{2}\left[\omega^{\nabla} \wedge \omega^{\nabla}\right]=\widetilde{\Omega}^{L C}+\tilde{d}^{L C} \widetilde{\eta}+\frac{1}{2}[\widetilde{\eta} \wedge \tilde{\eta}] .
$$

By Proposition 5.2, $\widetilde{\Omega}^{L C}=r^{2} \pi^{*}\left(\Omega^{L C}+\Omega_{\mathbb{P}^{n}}\right)$. For the same reasoning exposed in the proof of Proposition 7.1, $\tilde{d}^{L C} \tilde{\eta}=0$ if and only if $d^{L C} \eta-2 i s^{*} \varphi \wedge \eta=0$, which is granted by $\mathbf{D} 2$.

Finally, the computations leading to equation (10) still apply, and thus, we can deduce that

$$
\Omega^{\nabla}=r \pi^{*}\left(\Omega^{L C}+\Omega_{\mathbb{P}_{\mathbb{C}}^{n}}+[\eta \wedge \bar{\eta}]\right)=0
$$

making the connection $\nabla$ flat.

Notice that $\pi: \widetilde{M} \rightarrow M$ is a principal $\mathbb{C}^{*}$-bundle, where for all $l e^{i \theta} \in \mathbb{C}^{*}$ and $(u, t) \in \widetilde{M}$ :

$$
(u, t) l e^{i \theta}:=\left(u e^{i \theta}, t l\right) .
$$

The infinitesimal vector field corresponding to 1 at $\left(u, t_{0}\right)$ is $\xi_{\left(u, t_{0}\right)}$ and the one corresponding to $i$ is $X:=\left.\frac{d}{d t}\left(\left(u, t_{0}\right) \exp (i t)\right)\right|_{t=0}=\left.\frac{d}{d t}\left(u e^{i t}, t_{0}\right)\right|_{t=0}$, which is vertical and such that $\tilde{\varphi}(X)=\varphi\left(p_{*} X\right)=\varphi\left(\left.\frac{d}{d t}\left(u e^{i t}\right)\right|_{t=0}\right)=1$ and $d r(X)=0$. This means that $X=I \xi$ since $\widetilde{g}(X, \cdot)=-r^{2} \widetilde{\varphi}=-r I d r=I \xi^{b}$.

We are only left to prove that $M$ is the Kähler quotient or $\widetilde{M}$ with respect to the $\mathrm{U}(1)$ -action and in order to do so, notice that $\widetilde{\omega}(I \xi, \cdot)=-\widetilde{g}(\xi, \cdot)=r d r=d\left(\frac{r^{2}}{2}\right)$, so $\mu:=\frac{r^{2}}{2}$ is a moment map for $I \xi$. Notice that $\mu^{-1}\left(\frac{1}{2}\right)=S \times\{1\}$ and $S$ is a principal bundle so, by definition of $\widetilde{g}$ and $\widetilde{\omega}, S / \mathrm{U}(1)$ is isometric to $M$ and this ends the proof.

Remark 7.7 Starting from the family $\left\{\eta_{\alpha}\right\}_{\alpha}$, we can build a bundle map $\gamma: S \rightarrow M$ as long as the $\eta_{\alpha}$ 's are linked by the relation $\eta_{\alpha}=g_{\alpha, \beta}^{2} \eta_{\beta}$ where $g_{\alpha, \beta}$ is a cocycle defining $S$.

Remark 7.8 Let $(M, g, I)$ be a Kähler manifold, then if $H^{2}(M, \mathbb{Z})=0$, in particular, every complex line bundle and every circle bundle are trivial. Moreover, by de Rham's theorem, $H_{d R}^{2}(M)=H^{2}(M, \mathbb{R})=H^{2}(M, \mathbb{Z}) \otimes \mathbb{R}=0$, so in particular $\omega=d \lambda$ for some $\lambda \in \Omega^{1}(M)$.

Corollary 7.9 A Kähler $2 n$-manifold $(M, g, I, \omega)$ such that $H^{2}(M, \mathbb{Z})=0$, has a projective special Kähler structure if and only if there exists a section $\eta: M \rightarrow \sharp_{2} S_{3,0} M$ such that

D1 $^{*} \quad \Omega^{L C}+\Omega_{\mathbb{P}^{n}}+[\eta \wedge \bar{\eta}]=0 ;$

$\mathbf{D 2}^{*} \quad d^{L C} \eta=-4 i \lambda \wedge \eta$;

for some $\lambda \in \Omega^{1}(M)$ such that $d \lambda=\omega$.

Proof If $M$ has a projective special Kähler structure, then from Theorem 7.6 we obtain an $S^{1}$-bundle $p: S \rightarrow M$ and the map $\gamma: S \rightarrow \sharp_{2} S_{3,0} M$. Consider the corresponding line bundle $L=S \times_{\mathrm{U}(1)} \mathbb{C}$. As noted in Remark 7.8, we can assume $L=M \times \mathbb{C}$ and $S=M \times S^{1}$. In particular, there is a global section $s: M \rightarrow S$ and if we call $\eta=\gamma \circ s: M \rightarrow \sharp_{2} S_{3,0} M$, 
it is a global section satisfying the curvature equation thanks to Theorem 7.6. Defining $\lambda:=-\frac{1}{2} s^{*} \varphi$, we have $d \lambda=-\frac{1}{2} s^{*}\left(-2 \pi_{S}^{*} \omega\right)=\left(\pi_{S} s\right)^{*} \omega=\omega$, and thus, also the differential condition is satisfied by Theorem 7.6.

Conversely, by de Rham's Theorem, we have $\lambda \in \Omega^{1}(M)$ such that $d \lambda=\omega$. We define $\pi_{S}=\pi_{1}: S=M \times S^{1} \rightarrow M$ and choose as connection the form $\varphi=\pi_{2}^{*} d \vartheta-2 \pi_{S}^{*} \lambda$, where $d \vartheta$ is the fundamental 1 -form on $S^{1}=\mathrm{U}(1)$. Then, $d \varphi=0-2 \pi_{S}^{*} d \lambda=-2 \pi_{S}^{*} \omega$, so $S \rightarrow M$ has the desired curvature. Moreover, it is trivial, so we have a global section $s: M \rightarrow S$ mapping $p$ to $(p, 1)$.

Given $\eta: M \rightarrow \sharp_{2} S_{3,0} M$ as in the statement, we define $\gamma: S \rightarrow \sharp_{2} S_{3,0} M$ such that $\gamma(p, a):=a^{2} \eta(p)$ for all $p \in M$ and $a \in \mathrm{U}(1)$. Notice that $\gamma \circ s=\gamma(\cdot, 1)=\eta$, so the curvature equation of this corollary gives the curvature equation in Theorem 7.6 and the same is true for the differential condition, since $s^{*} \varphi=s^{*} \pi_{2}^{*} d \vartheta-2 s^{*} \pi_{S}^{*} \lambda=0-2 \lambda$. By Theorem 7.6, $M$ is thus projective special Kähler.

Remark 7.10 Instead of requiring a section $\eta$ as in Corollary 7.9, we could use a section $\sigma$ of $S_{3,0} M$ such that $\sharp_{2} \sigma=\eta$.

\section{Varying the projective special Kähler structure by a U(1)-valued function}

Theorem 7.6 allows to find a whole class of projective special Kähler structures from a given one, as shown in the following

Proposition 8.1 Let $(\pi: \widetilde{M} \rightarrow M, \nabla)$ be a projective special Kähler manifold, let $\gamma: S \rightarrow \#_{2} S_{3,0} M$ be its intrinsic deviance and $\varphi \in \Omega^{1}(S)$ the principal connection form on $\pi_{S}: S \rightarrow M$, then for all $\beta \in \mathcal{C}^{\infty}(M, \mathrm{U}(1))$ there is a new projective special Kähler structure $\left(\pi: \widetilde{M}^{\beta} \rightarrow M, \nabla^{\beta}\right)$ with intrinsic deviance $\gamma^{\beta}=\beta \gamma: S \rightarrow \sharp_{2} S_{3,0} M$ on the same bundle $S$, with principal connection form $\varphi^{\beta}=\pi_{S}^{*}\left(\frac{d \beta}{2 i \beta}\right)+\varphi$.

Proof We want to use Theorem 7.6, so consider the same bundle $\pi_{S}: S \rightarrow M$, but with the new connection form $\varphi^{\beta}$. Notice that $\varphi^{\beta}$ is a real form, in fact $\bar{\beta} \beta=1$, so

$$
0=\beta d \bar{\beta}+\bar{\beta} d \beta=\bar{\beta} \beta\left(\frac{d \bar{\beta}}{\bar{\beta}}+\frac{d \beta}{\beta}\right)=\left(\overline{\left(\frac{d \beta}{\beta}\right)}+\frac{d \beta}{\beta}\right)=2 \operatorname{Re}\left(\frac{d \beta}{\beta}\right),
$$

and thus, $\operatorname{Im}\left(\frac{d \beta}{2 i \beta}\right)=-\frac{1}{2} \operatorname{Re}\left(\frac{d \beta}{\beta}\right)=0$. Moreover $d \varphi^{\beta}=-\pi_{S}^{*}\left(\frac{d \beta \wedge d \beta}{\beta^{2}}\right)+d \varphi=d \varphi=-2 \pi^{*} \omega$, so condition 1 of Theorem 7.6 this is a compatible principal connection form. The bundle map $\gamma^{\beta}$ is still homogeneous of degree 2 . We are only left to prove the two conditions of point 3 , so consider a family of sections $\left\{\left(U_{\alpha}, s_{\alpha}\right)\right\}_{\alpha \in \mathcal{A}}$ corresponding to a trivialisation of $S$ and let $\eta_{\alpha}^{\beta}:=\gamma^{\beta} \circ s_{\alpha}=\beta \gamma \circ s_{\alpha}=\beta \eta_{\alpha}$. We thus have

$$
\begin{aligned}
d^{L C} \eta_{\alpha}^{\beta} & =d^{L C}\left(\beta \eta_{\alpha}\right)=d \beta \wedge \eta_{\alpha}+\beta 2 i s_{\alpha}^{*} \varphi \wedge \eta_{\alpha}=2 i\left(\frac{d \beta}{2 i \beta}+s_{\alpha}^{*} \varphi\right) \wedge e^{2 i \beta} \eta_{\alpha} \\
& =2 i s_{\alpha}^{*}\left(d \pi_{S}^{*}\left(\frac{d \beta}{2 i \beta}\right)+s_{\alpha}^{*} \varphi\right) \wedge \eta_{\alpha}^{\beta}=2 i s_{\alpha}^{*} \varphi^{\beta} \wedge \eta_{\alpha}^{\beta} .
\end{aligned}
$$


As for the curvature condition D1, it still holds because

$$
\left[\eta_{\alpha}^{\beta} \wedge \overline{\eta_{\alpha}^{\beta}}\right]=\left[\beta \eta_{\alpha} \wedge \overline{\beta \eta_{\alpha}}\right]=\left[\eta_{\alpha} \wedge \overline{\eta_{\alpha}}\right]
$$

These modified deviances do not always provide an entirely new projective special Kähler structure. Before entering into the details, we recall the following elementary result.

Lemma 8.2 Let $M$ be a smooth manifold and $G$ a Lie group with Lie algebra $\mathfrak{g}$ such that there is a smooth right action

$$
r: M \times G \longrightarrow M .
$$

Then, the differential of $r$ at a point $(x, a)$ is

$$
r_{*}(X, A)=\left(R_{a}\right)_{*}(X)+A^{\circ},
$$

for all $X \in T_{x} M, A \in \mathfrak{g}$, where $A^{\circ}$ denotes the fundamental vector field associated to $A$.

Proof See, e.g. [32, Ex. 27.4, p. 326].

We now present the following isomorphism result:

Proposition 8.3 In the setting of Proposition 8.1 , if moreover $\beta$ has a square root, meaning that $\beta=b^{2}$ for some $b: M \rightarrow \mathrm{U}(1)$, then the map

$$
m_{b}: S \longrightarrow S, \quad u \longmapsto u . b\left(\pi_{S}(u)\right)=R_{b\left(\pi_{S}(u)\right)}(u)
$$

induces a bundle isomorphism preserving connection and deviance, that is

$$
\varphi^{\beta}=m_{b}^{*}(\varphi), \quad \gamma^{\beta}=\gamma \circ m_{b} .
$$

In particular, if $\beta^{*}: \mathbb{R} \cong H_{d R}^{1}\left(S^{1}\right) \rightarrow H_{d R}^{1}(M)$ is the zero map, then $\beta$ has a square root.

Proof The preservation of $\gamma$ follows from its 2-homogeneity, since for all $u \in S$ :

$$
\gamma \circ m_{b}(u)=\gamma\left(u b\left(\pi_{S}(u)\right)\right)=b\left(\pi_{S}(u)\right)^{2} \gamma(u)=\left(\beta \circ \pi_{S}\right) \gamma(u)=\gamma^{\beta} .
$$

For the connection, we first compute the differential of $m_{b}$. Let $r: S \times \mathrm{U}(1) \rightarrow S$ be the principal right action, then we can see $m_{b}$ as $r \circ\left(\operatorname{id}_{S} \times\left(b \circ \pi_{S}\right)\right)$. The differential of $\left(\operatorname{id}_{S} \times\left(u \circ \pi_{S}\right)\right)$ is $\operatorname{id}_{T S} \times \pi_{S}^{*} d b$, where $d b$ has values in $\mathfrak{u}(1)=i \mathbb{R}$. Lemma 8.2 gives us the differential of the action. We have

$$
\left(\left(m_{b}\right)_{*}\right)_{u}=\left(R_{b \pi_{S}(u)}\right)_{*}+\left(d_{\pi_{S}(u)} b\right)^{\circ} .
$$

Now let us compute the pullback of $\varphi$, using the fact that $\varphi$ is right invariant and $d \beta=d b^{2}=2 b d b$ 


$$
\begin{aligned}
m_{b}^{*}(\varphi) & =\varphi \circ\left(m_{b}\right)_{*}=\varphi \circ\left(R_{b \pi_{S}(u)}\right)_{*}+\varphi\left(\left(d_{\pi_{S}(u)} b\right)^{\circ}\right)=R_{b \pi_{S}(u)}^{*} \varphi+\frac{1}{i b} d_{\pi_{S}(u)} b \\
& =\varphi+\frac{1}{i 2 b^{2}} d_{\pi_{S}(u)} \beta=\varphi+\frac{1}{i 2 \beta} d_{\pi_{S}(u)} \beta=\varphi^{\beta} .
\end{aligned}
$$

In order to prove the last statement, let $a: \mathrm{U}(1) \rightarrow \mathbb{C}$ be the standard identification of $\mathrm{U}(1)$ with the unit circle. Denote by $\psi$ the fundamental form of $\mathrm{U}(1)$, then we can write

$$
\psi=\frac{1}{i a} d a .
$$

Now let $\beta: M \rightarrow \mathrm{U}(1)$, and consider the pullback

$$
\beta^{*} \psi=\beta^{*}\left(\frac{1}{i a} d a\right)=\frac{1}{i \beta} d \beta
$$

We have $0=\beta^{*}: H_{d R}^{1}(\mathrm{U}(1)) \rightarrow H_{d R}^{1}(M)$, so in particular $\frac{1}{i \beta} d \beta$ is exact. Let $\lambda \in \mathcal{C}^{\infty}(M)$ be such that $d \lambda=\frac{1}{i \beta} d \beta$, then $e^{-i \lambda} \beta$ is a smooth function with image in U(1) and differential

$$
-i e^{i \lambda} \beta d \lambda+e^{i \lambda} d \beta=-\frac{i e^{i \lambda} \beta}{i \beta} d \beta+e^{i \lambda} d \beta=-e^{i \lambda} d \beta+e^{i \lambda} d \beta=0 .
$$

So up to a locally constant function $k$, we have $\beta=k e^{i \lambda}$. Without loss of generality, we can assume $k=1\left(\right.$ take $\left.\lambda^{\prime}=\lambda-i \log (k)\right)$. Then, let $b=e^{\frac{i \lambda}{2}}$ and $b^{2}=\beta$.

Remark 8.4 In the family of projective special Kähler structures constructed in Proposition 8.1 , if $H_{d R}^{1}(M)=0$, then there is a unique projective special Kähler structure on $M$ up to a natural notion of isomorphism.

Even when $H_{d R}^{1}(M) \neq 0$, we can say exactly when a function has a global square root by considering the following functional for all $p \in M$ :

$$
F_{\beta, p}: \pi_{1}(M, p) \longrightarrow \mathbb{R}, \quad \sigma \longmapsto \frac{1}{2 \pi} \int_{\sigma} \frac{1}{i \beta} d \beta .
$$

Notice that, in the notation above,

$$
\frac{1}{2 \pi} \int_{\sigma} \frac{1}{i \beta} d \beta=\frac{1}{2 \pi} \int_{\beta \circ \sigma} \frac{1}{i a} d a=\frac{1}{2 \pi} \int_{\beta \circ \sigma} \psi,
$$

so, $F$ has image in $\mathbb{Z}$.

Lemma 8.5 Let $M$ be a smooth manifold and $\beta: M \rightarrow \mathrm{U}(1)$, then there exists $b: M \rightarrow \mathrm{U}(1)$ such that $\beta=b^{2}$ if and only if for all $p \in M$, the functional

$$
\left[F_{\beta, p}\right]: \pi_{1}(M, p) \longrightarrow \mathbb{Z}_{2}, \quad \sigma \longmapsto \frac{1}{2 \pi} \int_{\sigma} \frac{1}{i \beta} d \beta \bmod 2
$$

is zero. Explicitly, given $y_{p} \in \mathrm{U}(1)$ such that $y_{p}^{2}=\beta(p)$, then for all $q \in M$ in the same connected component of $p$, 


$$
b(q)=y_{p} \exp \left(\frac{1}{2} \int_{\sigma} \frac{1}{\beta} d \beta\right)
$$

for all continuous $\sigma:[0,1] \rightarrow M$ such that $\sigma(0)=p$ and $\sigma(1)=q$.

Proof If $\beta=b^{2}$ for some $b: M \rightarrow \mathrm{U}(1)$, then for all $p \in M$ and $\sigma \in \pi_{1}(M, p)$,

$$
F_{\beta, p}(\sigma)=\frac{1}{2 \pi} \int_{\sigma} \frac{2}{i b} d b=2\left(\frac{1}{2 \pi} \int_{b \circ \sigma} \psi\right) .
$$

Since $b \circ \sigma$ is a loop, $F_{\beta, p}(\sigma)$ is even, so $\left[F_{\beta, p}\right]=0$.

Conversely, choose a point in every connected component of $M$ and define $b$ by glueing functions defined as in (15). We can verify $\beta=b^{2}$ on each connected component, so let $p$ be the chosen point in said component. Connected components on manifolds are also path connected components, so for all $q$ in the same connected component, there exists a smooth $\sigma:[0,1] \rightarrow M$ such that $\sigma(0)=p$ and $\sigma(1)=q$. The value $b(q)$ is independent from the path $\sigma$ chosen, in fact if we pick another such $\sigma^{\prime}:[0,1] \rightarrow M$, then the composition of paths $\left(\sigma^{\prime}\right)^{-1} * \sigma \in \pi_{1}(M, p)$ is a loop, and thus

$$
\int_{\sigma} \frac{1}{\beta} d \beta-\int_{\sigma^{\prime}} \frac{1}{\beta} d \beta=\int_{\sigma} \frac{1}{\beta} d \beta+\int_{\left(\sigma^{\prime}\right)^{-1}} \frac{1}{\beta} d \beta=2 \pi i\left(\frac{1}{2 \pi} \int_{\left(\sigma^{\prime}\right)^{-1} * \sigma} \frac{1}{i \beta} d \beta\right)=4 \pi i k
$$

for some $k \in \mathbb{Z}$. It follows that

$$
y_{p} \exp \left(\frac{1}{2} \int_{\sigma} \frac{1}{\beta} d \beta\right)=y_{p} \exp \left(\frac{1}{2} \int_{\sigma^{\prime}} \frac{1}{\beta} d \beta+2 \pi i k\right)=y_{p} \exp \left(\frac{1}{2} \int_{\sigma^{\prime}} \frac{1}{\beta} d \beta\right)
$$

We can now compute

$$
b^{2}(q)=y_{p}^{2}\left(\exp \left(\frac{1}{2} \int_{\sigma} \frac{1}{\beta} d \beta\right)\right)^{2}=\beta(p) \exp \left(\int_{\sigma} \frac{1}{\beta} d \beta\right) .
$$

Since locally $\frac{1}{\beta} d \beta=d \log (\beta)$, up to picking a suitable partition of $[0,1]$ we have $\exp \left(\int_{\sigma} \frac{1}{\beta} d \beta\right)=\beta(q) / \beta(p)$ so $b^{2}(q)=\beta(q)$.

We deduce

Proposition 8.6 Let $M$ be a smooth manifold and $\beta: M \rightarrow \mathrm{U}(1)$, then the following are equivalent:

1. there exists $b: M \rightarrow \mathrm{U}(1)$ such that $\beta=b^{2}$;

2. $\left[F_{\beta, p}\right]=0$ for all $p \in M$;

3. $\left[F_{\beta, p_{k}}\right]\left(\sigma_{k}\right)=0$ for a set of loops $\sigma_{k} \in \pi_{1}\left(M, p_{k}\right)$ corresponding to a set of generators of $H_{1}(M, \mathbb{Z})$;

4. $\left[F_{\beta, p_{k}}\right]\left(\sigma_{k}\right)=0$ for a set of loops $\sigma_{k} \in \pi_{1}\left(M, p_{k}\right)$ corresponding to a set of generators of $H_{1}\left(M, \mathbb{Z}_{2}\right)=H_{1}(M, \mathbb{Z}) \otimes_{\mathbb{Z}} \mathbb{Z}_{2} ;$

5. the pullback $\beta^{*}: \mathbb{Z}_{2} \cong H^{1}\left(\mathrm{U}(1), \mathbb{Z}_{2}\right) \rightarrow H^{1}\left(M, \mathbb{Z}_{2}\right)$ is the zero map. 
Proof The equivalence $1 \Leftrightarrow 2$ is Lemma 8.5.

For $2 \Leftrightarrow 3$, suppose at first that $M$ is connected and let $p \in M$. The functional $\left[F_{\beta, p}\right]: \pi_{1}(M, p) \rightarrow \mathbb{Z}_{2}$ is a group homomorphism and by Hurewicz theorem, $H_{1}(M, \mathbb{Z})$ is the abelianisation of $\pi_{1}(M, p)$. Since $\mathbb{Z}_{2}$ is an abelian group, there are natural isomorphisms

$$
\operatorname{Hom}\left(\pi(M, p), \mathbb{Z}_{2}\right) \cong \operatorname{Hom}\left(H_{1}(M, \mathbb{Z}), \mathbb{Z}_{2}\right)=\operatorname{Hom}_{\mathbb{Z}}\left(H_{1}(M, \mathbb{Z}), \mathbb{Z}_{2}\right),
$$

and thus, there is a canonical factorisation of $\left[F_{\beta, p}\right]$ as an abelian group homomorphism (i.e. $\mathbb{Z}$-linear map) $H_{1}(M, \mathbb{Z}) \rightarrow \mathbb{Z}_{2}$ which is the zero map if and only if $\left[F_{\beta, p}\right]$ is zero. In particular, this proves $2 \Leftrightarrow 3$. In general, $M=\coprod_{i \in I} M_{i}$ with $M_{i}$ connected for all $i \in I$, so $H_{1}(M, \mathbb{Z})=\bigoplus_{i \in I} H_{1}\left(M_{i}, \mathbb{Z}\right)$ and hence

$$
\operatorname{Hom}_{\mathbb{Z}}\left(H_{1}(M, \mathbb{Z}), \mathbb{Z}_{2}\right) \cong \prod_{i \in I} \operatorname{Hom}_{\mathbb{Z}}\left(H_{1}\left(M_{i}, \mathbb{Z}\right), \mathbb{Z}_{2}\right) \cong \prod_{i \in I} \operatorname{Hom}\left(\pi_{1}\left(M_{i}, p_{i}\right), \mathbb{Z}_{2}\right) .
$$

Thus, 2 holds if and only if $\left[F_{\beta, p_{i}}\right]=0$ for all $i \in I$, and by the previous isomorphism, this happens if and only if 3 .

$3 \Leftrightarrow 4$ follows from properties of tensor products and linear maps, in fact, given a $\mathbb{Z}$-module $A$, a $\mathbb{Z}$-linear map $A \rightarrow \mathbb{Z}_{2}$ vanishes on $2 A$, and thus, factors as a map $A / 2 A \rightarrow \mathbb{Z}_{2}$. Moreover, $A / 2 A \cong A \otimes_{\mathbb{Z}} \mathbb{Z}_{2}$ (seen by applying the right-exact functor $A \bigotimes_{\mathbb{Z}} \cdot$ to the short exact sequence $0 \rightarrow \mathbb{Z} \stackrel{2 \cdot}{\longrightarrow} \mathbb{Z} \rightarrow \mathbb{Z}_{2} \rightarrow 0$ ). From these properties along with the homological universal coefficients theorem, we find the following natural isomorphisms

$$
\operatorname{Hom}_{\mathbb{Z}}\left(H_{1}(M, \mathbb{Z}), \mathbb{Z}_{2}\right) \cong \operatorname{Hom}_{\mathbb{Z}_{2}}\left(H_{1}(M, \mathbb{Z}) \otimes_{\mathbb{Z}} \mathbb{Z}_{2}, \mathbb{Z}_{2}\right) \cong \operatorname{Hom}_{\mathbb{Z}_{2}}\left(H_{1}\left(M, \mathbb{Z}_{2}\right), \mathbb{Z}_{2}\right),
$$

that show the equivalence $3 \Leftrightarrow 4$.

Finally, we prove $3 \Leftrightarrow 5$ by the cohomological universal coefficient theorem, which gives the natural isomorphism $\operatorname{Hom}\left(H_{1}(M, \mathbb{Z}), \mathbb{Z}_{2}\right) \cong H^{1}\left(M, \mathbb{Z}_{2}\right)$. In particular, the class in $H^{1}\left(M, \mathbb{Z}_{2}\right)$ corresponding to $\left[F_{\beta, p}\right\rfloor$, is by construction the pullback along $\beta$ of the fundamental form on $\mathrm{U}(1)$ (see (14)). Since $H^{1}\left(\mathrm{U}(1), \mathbb{Z}_{2}\right)$ is generated by the integral functional associated to the fundamental form, this image is zero if and only if the whole $\beta^{*}$ is the zero map.

This proposition clarifies when two structures built as in Proposition 8.1 are isomorphic as in Proposition 8.3. Since $\beta^{*}$ always vanishes on torsion elements, $H_{d R}^{1}(M)=0$ is a sufficient condition for not only $\beta^{*}: H_{d R}^{1}(\mathrm{U}(1)) \rightarrow H_{d R}^{1}(M)$ being zero, but also for $\beta^{*}: H^{1}\left(\mathrm{U}(1), \mathbb{Z}_{2}\right) \rightarrow H^{1}\left(M, \mathbb{Z}_{2}\right)$ being zero. However, the condition $\beta^{*}=0$ on the cohomology with coefficients in $\mathbb{Z}_{2}$ is in general strictly weaker than the same condition in de Rham cohomology.

\section{Complex hyperbolic n-space}

In this section, we are going to describe a special family of projective special Kähler manifolds, which can be thought of as the simplest possible model in a given dimension.

Let $\mathbb{C}^{n, 1}$ be the Hermitian space $\mathbb{C}^{n+1}$ endowed with the Hermitian form

$$
\langle z, w\rangle=\overline{z_{1}} w_{1}+\cdots+\overline{z_{n}} w_{n}-\overline{z_{n+1}} w_{n+1} .
$$

It is a complex vector space, so it makes sense to consider the projective space associated to it, that is $\mathbb{P}\left(\mathbb{C}^{n, 1}\right)=\left(\mathbb{C}^{n, 1} \backslash\{0\}\right) / \mathbb{C}^{*}$ with the quotient topology and the canonical 
differentiable structure, where $\mathbb{C}^{*}$ acts by scalar multiplication. We will denote the quotient class corresponding to an element $z \in \mathbb{C}^{n, 1}$ by $[z]$. We can define the following open subset:

$$
\mathcal{H}_{\mathbb{C}}^{n}:=\left\{[v] \in \mathbb{P}\left(\mathbb{C}^{n, 1}\right) \mid\langle v, v\rangle<0\right\} .
$$

Let $v=\left(v_{1}, \ldots, v_{n+1}\right) \in \mathbb{C}^{n, 1}$, notice that if $[v] \in \mathcal{H}_{\mathbb{C}}^{n}$, then $\left|v_{1}\right|^{2}+\cdots+\left|v_{n}\right|^{2}-\left|v_{n+1}\right|^{2}<0$ so $\left|v_{n+1}\right|^{2}>\left|v_{1}\right|^{2}+\cdots+\left|v_{n}\right|^{2} \geq 0$ which implies $v_{n+1} \neq 0$. We thus have a global differentiable chart $\mathcal{H}_{\mathbb{C}}^{n} \rightarrow \mathbb{C}^{n}$ by restricting the projective chart $[v] \mapsto\left(\frac{v_{1}}{v_{n+1}}, \ldots, \frac{v_{n}}{v_{n+1}}\right)$.

Remark 9.1 The inverse of this chart $\mathbb{C}^{n} \rightarrow \mathbb{P}\left(\mathbb{C}^{n, 1}\right)$ maps $z=\left(z_{1}, \ldots, z_{n}\right) \in \mathbb{C}^{n}$ to $\left[\left(z_{1}, \ldots, z_{n}, 1\right)\right]$, which is in $\mathcal{H}_{\mathbb{C}}^{n}$ if and only if $\|z\|^{2}<1$. We have proven that $\mathcal{H}_{\mathbb{C}}^{n}$ is diffeomorphic to the complex unit ball, and thus, in particular it is contractible.

Consider now the Lie group $\operatorname{SU}(n, 1)$ of the matrices with determinant 1 that are unitary with respect to the Hermitian metric on $\mathbb{C}^{n, 1}$. We define a left action of $\operatorname{SU}(n, 1)$ on $\mathcal{H}_{\mathbb{C}}$ such that $A[v]=[A v]$; it is well defined by linearity and invertibility and it is smooth.

This action is also transitive, in fact given $[v],[w] \in \mathcal{H}_{\mathbb{C}}^{n}$, without loss of generality, we can assume that $\langle v, v\rangle=-1=\langle w, w\rangle$. Because of this, we can always complete $v$ and $w$ to an orthonormal basis with respect to the Hermitian product, obtaining $\left\{v_{1}, \ldots, v_{n}, v\right\}$ and $\left\{w_{1}, \ldots, w_{n}, w\right\}$. Consider the following block matrices $V=\left(v_{1}|\ldots| v_{n} \mid v\right)$ and $W=\left(w_{1}|\ldots| w_{n} \mid w\right)$ which, up to permuting two of the first $n$-columns, belong to $\operatorname{SU}(n, 1)$. The matrix $A=W V^{-1} \in \mathrm{SU}(n, 1)$ maps $v$ in $w$ and thus $[v]$ in $[w]$.

We shall now compute the stabiliser of the last element of the canonical basis $e_{n+1}$ for this action, that is, the set of matrices $A \in \mathrm{SU}(n, 1)$ such that $A e_{n+1}=\lambda e_{n+1}$ for $\lambda \in \mathbb{C}$. Observe that $\lambda \in \mathrm{U}(1)$ since

$$
-1=\left\langle e_{n+1}, e_{n+1}\right\rangle=\left\langle A e_{n+1}, A e_{n+1}\right\rangle=\left\langle\lambda e_{n+1}, \lambda e_{n+1}\right\rangle=-|\lambda|^{2} .
$$

Moreover, the last column of $A$ is $A_{n+1}=A e_{n+1}=\lambda e_{n+1}$. This forces $A$ to assume the form

$$
\left(\begin{array}{ll}
B & 0 \\
0 & \lambda
\end{array}\right) \text {. }
$$

Since $A$ belongs to $\mathrm{SU}(n, 1)$, we must infer that $B$ belongs to $\mathrm{U}(n)$ and $\lambda=\operatorname{det}(B)^{-1}$. The stabiliser of $e_{n+1}$ is thus $\mathrm{S}(\mathrm{U}(n) \mathrm{U}(1))$, which is isomorphic to $\mathrm{U}(n)$. We deduce that $\mathcal{H}_{\mathbb{C}}^{n}$ is a symmetric space $\mathrm{SU}(n, 1) / \mathrm{S}(\mathrm{U}(n) \mathrm{U}(1))$.

We will adopt the nomenclature of [21] for the following

Definition 9.2 We call the Kähler manifold $\mathcal{H}_{\mathbb{C}}^{n}$ of complex dimension $n$ the complex hyperbolic n-space.

There is a natural Kähler structure on $\mathcal{H}_{\mathbb{C}}^{n}$ coming from its representation as a symmetric space $G / H$.

Let $\mathfrak{g}=\mathfrak{h}+\mathfrak{m}$ be the canonical decomposition, in particular

$$
\mathfrak{m}:=\left\{\left(\begin{array}{cc}
0 & x \\
x^{\star} & 0
\end{array}\right) \mid x \in \mathbb{C}^{n}\right\} .
$$

On a symmetric space, there is a one-to-one correspondence between Riemannian metrics and $\operatorname{Ad}(H)$-invariant positive definite symmetric bilinear forms on $\mathfrak{m}$ (See [24, II, Corollary 
3.2, p. 200]). Let $\theta: T_{\left[e_{n+1}\right]} \mathcal{H}_{\mathbb{C}}^{n} \cong \mathfrak{m} \rightarrow \mathbb{C}^{n}$ be the identification mapping to $x$ the tangent vector corresponding to $\left(\begin{array}{cc}0 & x \\ x^{\star} & 0\end{array}\right)$. With this identification, for $A \in \mathrm{U}(n)$ we see that the $\operatorname{Ad}(A)$-action on $\mathfrak{m}$ corresponds on $\mathbb{C}^{n}$ to the $x \mapsto \operatorname{det}(A) A x$.

The metric is induced by the Killing form on $\mathfrak{g} \mathfrak{t}(n, 1)$ given by ([22])

$$
B(X, Y)=2(n+1) \operatorname{tr}(X Y), \quad \forall X, Y \in \mathfrak{t}(n, 1) .
$$

We restrict the Killing form to $\mathfrak{m}$ in order to define an $\operatorname{Ad}(H)$-invariant bilinear form, that is, given $x, y \in \mathbb{C}^{n}$, if $X, Y$ are the corresponding tangent vectors,

$$
\begin{aligned}
B(X, Y) & =2(n+1) \operatorname{tr}\left(\left(\begin{array}{cc}
0 & x \\
x^{\star} & 0
\end{array}\right)\left(\begin{array}{cc}
0 & y \\
y^{\star} & 0
\end{array}\right)\right)=2(n+1) \operatorname{tr}\left(\begin{array}{cc}
x y^{\star} & 0 \\
0 & x^{\star} y
\end{array}\right) \\
& =2(n+1) \operatorname{Re}\left(x^{\star} y\right)=2(n+1)\left(\theta^{\star} \theta\right)(X, Y) .
\end{aligned}
$$

We define $g_{\left[e_{n+1}\right]}:=\theta^{\star} \theta$, which is $\operatorname{Ad}(\mathrm{U}(n))$-invariant, so it extends to a global Riemannian metric $g$. By using the same idea, we can also define an almost complex structure $I$ on $\mathfrak{m}$ as the map corresponding to the scalar multiplication by $i$ on $\mathbb{C}^{n}$. This structure is compatible with the metric and it is $\operatorname{Ad}(\mathrm{U}(n)$ )-invariant, so it defines a Kähler structure (see [24, II, Proposition 9.3, p. 260]). The Kähler form $\omega$ is then:

$$
\omega(X, Y)=g(I X, Y)=\operatorname{Re}\left(x^{\star} i^{\star} y\right)=\operatorname{Im}\left(x^{\star} y\right)=\operatorname{Im}\left(\theta^{\star} \otimes \theta\right)(X, Y) .
$$

Proposition 9.3 The manifold $\mathcal{H}_{\mathbb{C}}^{n}$ has curvature tensor $-\Omega_{\mathbb{P}_{\mathbb{C}}^{n}}$ and is projective special Kähler for all $n \geq 1$ with constant zero deviance.

Proof The computation of the curvature tensor is standard. By Remark 9.1, we know that $\mathcal{H}_{\mathbb{C}}^{n}$ is contractible, allowing us to apply Corollary 7.9. If we choose as tensor $\eta$ of type $\#_{2} S_{3,0} M$ the 0 -section, then the differential condition D2 is trivially satisfied, while condition D1 follows from the computation of the curvature tensor.

Notice that the deviance measures the difference of a projective special Kähler manifold of dimension $2 n$ from being the complex hyperbolic $n$-space. More precisely, we have

Proposition 9.4 At a point $p$ of a projective special Kähler manifold $M$ with intrinsic deviance $\gamma: S \rightarrow \sharp_{2} S_{3,0} M$, the curvature tensor $\Omega_{M}$ coincides with the one of $\mathcal{H}_{\mathbb{C}}^{n}$ exactly in those points $p$ where $\left.\gamma\right|_{p}$ vanishes.

In particular, for any section of $S$ defined on an open neighbourhood of $p$, the corresponding local deviance vanishes at $p$ whenever the two curvatures coincide.

Proof One direction follows from condition D1. For the opposite one, if $\Omega_{M}=\Omega_{\mathcal{H}_{\mathrm{C}}^{n}}=-\Omega_{\mathbb{P}_{\mathrm{C}}^{n}}$, then $\operatorname{scal}_{M}=-2(n+1)$ and the intrinsic deviance vanishes as the norm of any local deviance vanishes by (13).

We can also prove 
Proposition 9.5 The only complete connected and simply connected projective special Kähler manifold of dimension $2 n$ with zero deviance is $\mathcal{H}_{\mathbb{C}}^{n}$.

Proof Let $(\pi: \widetilde{M} \rightarrow M, \nabla)$ be such a projective special Kähler manifold. Consider a point $p \in M$, then $\left(T_{p} M, g, I\right)$ can be seen as a complex vector space compatible with the metric and can thus be identified with the tangent space at a point of $\mathcal{H}_{\mathbb{C}}^{n}$ via an isomorphism $F$ as they are both isomorphic to $\mathbb{C}^{n}$ with the standard metric. Being complex manifolds, $\mathcal{H}_{\mathbb{C}}^{n}$ and $M$ are analytic, and since the curvature of $M$ is forced to be $-\Omega_{\mathbb{P}_{c}^{n}}$, which corresponds to a $\mathfrak{t}(n)$-invariant map from the bundle of unitary frames to $S^{2}(\mathfrak{t}(n))$, it is also parallel with respect to the Levi-Civita connection. It follows that the linear isomorphism $F$ preserves the curvature tensors and their covariant derivatives. Thus, $F$ can be extended to a diffeomorphism $f: M \rightarrow \mathcal{H}_{\mathbb{C}}^{n}($ See $[24$, I, Corollary 7.3, p. 261]) such that $F$ is its differential at $p$.

Since $F$ preserves $I$ and $\omega$ which are parallel, $f$ is an isomorphism of Kähler manifolds, as the latter maps parallel tensors to parallel tensors. Since the deviance of both manifolds is zero, we also have an isomorphism of projective special Kähler manifolds.

\section{Classification of projective special Kähler Lie groups in dimension 4}

If $M$ is a Lie group, the conditions of Theorem 7.6 are simpler, because a Lie group is always parallelisable. As a consequence, the bundle $\sharp_{2} S_{3,0}(M)$ is trivial, and in particular we have a global coordinate system to write the local deviances.

Definition 10.1 A projective special Kähler Lie group is a Lie group with projective special Kähler structure such that the Kähler structure is left-invariant.

Notice that we do not require the deviance to be left-invariant.

An example is $\mathcal{H}_{\mathbb{C}}^{n}$, since the Iwasawa decomposition $\mathrm{SU}(n, 1)=K A N$ (see [22, Theorem 1.3, p. 403]) gives a left-invariant Kähler structure on the solvable Lie group $A N$. We denote by $\mathcal{H}_{\lambda}$ the hyperbolic plane with curvature $-\lambda^{2}$, which is actually just a rescaling of $\mathcal{H}_{\mathbb{C}}^{1}$

With Definition 10.1, we are able to classify 4-dimensional projective special Kähler Lie groups; we obtain exactly two, which coincide with the two 4-dimensional cases appearing in the classification of projective special Kähler manifolds homogeneous under the action of a semisimple Lie groups ([1]).

Theorem 10.2 Up to isomorphisms of projective special Kähler manifolds, there are only two 4-dimensional connected and simply connected projective special Kähler Lie groups: $\mathcal{H}_{\sqrt{2}} \times \mathcal{H}_{2}$ and the complex hyperbolic plane.

Up to isomorphisms that also preserve the Lie group structure, there are four families of 4-dimensional connected and simply connected projective special Kähler Lie groups, listed in Table 1. 
Table 1 Connected projective special Kähler Lie groups

\begin{tabular}{llll}
\hline PSK & Diff. complex unitary cof. $\theta$ & Riemann curv. & $\sigma$ \\
\hline $\mathcal{H}_{\sqrt{2}} \times \mathcal{H}_{2}$ & $d \theta^{1}=-\frac{\sqrt{2}}{\overline{2}} \overline{\theta^{1}} \wedge \theta^{1}$ & $\left.\frac{1}{2} \overline{\theta^{1}} \wedge \theta^{1}\right)^{2}+\left(\overline{\theta^{2}} \wedge \theta^{2}\right)^{2}$ & $\frac{3}{2}\left(\theta^{1}\right)^{2} \theta^{2}$ \\
& $d \theta^{2}=-\overline{\theta^{2}} \wedge \theta^{2}$ & \\
$\mathcal{H}_{\mathbb{C}}^{2}$ & $d \theta^{1}=\frac{1}{2} \theta^{1} \wedge\left(\theta^{2}+\overline{\theta^{2}}\right)$ & $-\Omega_{\mathbb{P}_{\mathbb{C}}^{b}}^{b}$ & \\
& $d \theta^{2}=-\overline{\theta^{1}} \wedge \theta^{1}-\overline{\theta^{2}} \wedge \theta^{2}$ & $-\frac{1}{\delta} \Omega_{\mathbb{P}_{\mathbb{C}}^{b}}^{b}$ & \\
$\mathcal{H}_{\mathbb{C}}^{2}$ & $d \theta^{1}=\left(\frac{1}{2}-\frac{i}{\delta}\right) \theta^{1} \wedge\left(\theta^{2}+\overline{\theta^{2}}\right)$ & & \\
& $d \theta^{2}=-\overline{\theta^{1}} \wedge \theta^{1}-\overline{\theta^{2}} \wedge \theta^{2}$ & \\
& $\delta>0$ & $-\frac{1}{\delta} \Omega_{\mathbb{P}_{\mathbb{C}}^{b}}^{b}$ & \\
$\mathcal{H}_{\mathbb{C}}^{2}$ & $d \theta^{1}=-\left(\frac{1}{\delta}+\frac{i}{2}\right) \theta^{1} \wedge\left(\theta^{2}-\overline{\theta^{2}}\right)$ & \\
& $d \theta^{2}=-i \overline{\theta^{1}} \wedge \theta^{1}-\overline{\theta^{2}} \wedge \theta^{2}$ & \\
& $\delta>0$ & &
\end{tabular}

Proof We will study Kähler Lie groups through Kähler Lie algebras, see, e.g. [17, §1.1, p. 26]. We will start from the classification of pseudo-Kähler Lie groups provided by [28]. Table 2 displays the eighteen families of non-abelian pseudo-Kähler Lie algebras in dimension 4.

Among these families, only for the ones in Table 3 the metric can be positive definite, i.e. Kähler.

Table 2 Classification of 4-dimensional pseudo-Kähler non-abelian Lie algebras [28, Table 5.1, p. 63]

\begin{tabular}{|c|c|c|c|}
\hline $\mathfrak{g}$ & $I$ & $\omega$ & Conditions \\
\hline $\mathfrak{r h}_{3}$ & $I e_{1}=e_{2}, I e_{3}=e_{4}$ & $a_{1}\left(e^{13}+e^{24}\right)+a_{2}\left(e^{14}-e^{23}\right)+a_{3} e^{12}$ & $a_{1}^{2}+a_{2}^{2} \neq 0$ \\
\hline $\mathfrak{r r}_{3,0}$ & $I e_{1}=e_{2}, I e_{3}=e_{4}$ & $a_{1} e^{12}+a_{2} e^{34}$ & $a_{1} a_{2} \neq 0$ \\
\hline $\mathfrak{r} \mathfrak{r}_{3,0}^{\prime}$ & $I e_{1}=e_{4}, I e_{2}=e_{3}$ & $a_{1} e^{14}+a_{2} e^{23}$ & $a_{1} a_{2} \neq 0$ \\
\hline $\mathfrak{r}_{2} \mathfrak{r}_{2}$ & $I e_{1}=e_{2}, I e_{3}=e_{4}$ & $a_{1} e^{12}+a_{2} e^{34}$ & $a_{1} a_{2} \neq 0$ \\
\hline $\mathfrak{r}_{2}^{\prime}$ & $I e_{1}=e_{3}, I e_{2}=e_{4}$ & $a_{1}\left(e^{13}-e^{24}\right)+a_{2}\left(e^{14}+e^{23}\right)$ & $a_{1}^{2}+a_{2}^{2} \neq 0$ \\
\hline $\mathfrak{r}_{2}^{\prime}$ & $I e_{1}=-e_{2}, I e_{3}=e_{4}$ & $a_{1}\left(e^{13}-e^{24}\right)+a_{2}\left(e^{14}+e^{23}\right)+a_{3} e^{12}$ & $a_{1}^{2}+a_{2}^{2} \neq 0$ \\
\hline $\mathfrak{r}_{4,-1,-1}$ & $I e_{4}=e_{1}, I e_{2}=e_{3}$ & $a_{1}\left(e^{12}+e^{34}\right)+a_{2}\left(e^{13}-e^{24}\right)+a_{3} e^{14}$ & $a_{1}^{2}+a_{2}^{2} \neq 0$ \\
\hline $\mathfrak{r}_{4,0, \delta}^{\prime}$ & $I e_{4}=e_{1}, I e_{2}=e_{3}$ & $a_{1} e^{14}+a_{2} e^{23}, \quad a_{1} a_{2} \neq 0$ & $\delta>0$ \\
\hline $\mathfrak{r}_{4,0, \delta}^{\prime}$ & $I e_{4}=e_{1}, I e_{2}=-e_{3}$ & $a_{1} e^{14}+a_{2} e^{23}, \quad a_{1} a_{2} \neq 0$ & $\delta>0$ \\
\hline $\mathfrak{D}_{4,1}$ & $I e_{1}=e_{4}, I e_{2}=e_{3}$ & $a_{1}\left(e^{12}-e^{34}\right)+a_{2} e^{14}$ & $a_{1} \neq 0$ \\
\hline $\mathfrak{d}_{4,2}$ & $I e_{4}=-e_{2}, I e_{1}=e_{3}$ & $a_{1}\left(e^{14}+e^{23}\right)+a_{2} e^{24}$ & $a_{1} \neq 0$ \\
\hline $\mathfrak{b}_{4,2}$ & $I e_{4}=-2 e_{1}, I e_{2}=e_{3}$ & $a_{1} e^{14}+a_{2} e^{23}$ & $a_{1} a_{2} \neq 0$ \\
\hline $\mathfrak{d}_{4,1 / 2}$ & $I e_{4}=e_{3}, I e_{1}=e_{2}$ & $a_{1}\left(e^{12}-e^{34}\right)$ & $a_{1} \neq 0$ \\
\hline $\mathfrak{d}_{4,1 / 2}$ & $I e_{4}=e_{3}, I e_{1}=-e_{2}$ & $a_{1}\left(e^{12}-e^{34}\right)$ & $a_{1} \neq 0$ \\
\hline $\mathfrak{D}_{4, \delta}^{\prime}$ & $I e_{4}=e_{3}, I e_{1}=e_{2}$ & $a_{1}\left(e^{12}-\delta e^{34}\right)$ & $a_{1} \neq 0$ \\
\hline $\mathfrak{d}_{4, \delta}^{\prime \prime}$ & $I e_{4}=-e_{3}, I e_{1}=e_{2}$ & $a_{1}\left(e^{12}-\delta e^{34}\right)$ & $a_{1} \neq 0, \quad \delta>0$ \\
\hline $\mathfrak{d}_{4, \delta}^{\prime}$ & $I e_{4}=-e_{3}, I e_{1}=-e_{2}$ & $a_{1}\left(e^{12}-\delta e^{34}\right)$ & $a_{1} \neq 0, \quad \delta>0$ \\
\hline $\mathfrak{D}_{4, \delta}^{\prime}$ & $I e_{4}=e_{3}, I e_{1}=-e_{2}$ & $a_{1}\left(e^{12}-\delta e^{34}\right)$ & $a_{1} \neq 0, \quad \delta>0$ \\
\hline
\end{tabular}


Table 3 Non-abelian Kähler Lie algebras of dimension 4

\begin{tabular}{lllll}
\hline Case & $\mathfrak{g}$ & $I$ & $\omega$ & Conditions \\
\hline $\mathrm{I}$ & $\mathfrak{r r}_{3,0}$ & $I e_{1}=e_{2}, I e_{3}=e_{4}$ & $a_{1} e^{12}+a_{2} e^{34}$ & $a_{1}, a_{2}>0$ \\
$\mathrm{II}$ & $\mathfrak{r r}_{3,0}^{\prime}$ & $I e_{1}=e_{4}, I e_{2}=e_{3}$ & $a_{1} e^{14}+a_{2} e^{23}$ & $a_{1}, a_{2}>0$ \\
$\mathrm{III}$ & $\mathfrak{r}_{2} \mathfrak{r}_{2}$ & $I e_{1}=e_{2}, I e_{3}=e_{4}$ & $a_{1} e^{12}+a_{2} e^{34}$ & $a_{1}, a_{2}>0$ \\
$\mathrm{IV}$ & $\mathfrak{r}_{4,0, \delta}^{\prime}$ & $I e_{4}=e_{1}, I e_{2}=e_{3}$ & $a_{1} e^{14}+a_{2} e^{23}$ & $a_{1}<0 ; a_{2}, \delta>0$ \\
$\mathrm{~V}$ & $\mathfrak{r}_{4,0, \delta}^{\prime}$ & $I e_{4}=e_{1}, I e_{2}=-e_{3}$ & $a_{1} e^{14}+a_{2} e^{23}$ & $a_{1}, a_{2}<0 ; \delta>0$ \\
$\mathrm{VI}$ & $\mathfrak{D}_{4,2}$ & $I e_{4}=-2 e_{1}, I e_{2}=e_{3}$ & $a_{1} e^{14}+a_{2} e^{23}$ & $a_{1}, a_{2}>0$ \\
$\mathrm{VII}$ & $\mathfrak{D}_{4,1 / 2}$ & $I e_{4}=e_{3}, I e_{1}=e_{2}$ & $a_{1}\left(e^{12}-e^{34}\right)$ & $a_{1}>0$ \\
$\mathrm{VIII}$ & $\mathfrak{D}_{4, \delta}^{\prime}$ & $I e_{4}=e_{3}, I e_{1}=e_{2}$ & $a_{1}\left(e^{12}-\delta e^{34}\right)$ & $a_{1}, \delta>0$ \\
$\mathrm{IX}$ & $\mathfrak{b}_{4, \delta}^{\prime}$ & $I e_{4}=-e_{3}, I e_{1}=-e_{2}$ & $a_{1}\left(e^{12}-\delta e^{34}\right)$ & $a_{1}<0 ; \delta>0$ \\
\hline & & & &
\end{tabular}

It is now straightforward to find a unitary frame $u$ for each case, that is such that $g=\sum_{k=1}^{4}\left(u^{k}\right)^{2}, I u_{1}=u_{2}, I u_{3}=u_{4}$ and $\omega=u^{1,2}+u^{3,4}$. With respect to $u$, we can write the new structure constants and compute the Levi-Civita connection form $\omega^{L C}$ and the corresponding curvature forming its generic form with respect $\Omega^{L C}$. We write

$$
H_{1}:=\left(\begin{array}{ll}
u^{12} & -u^{12} \\
\hline &
\end{array}\right), \quad H_{2}=\left(\begin{array}{l}
\mid \\
u^{34}
\end{array}\right) .
$$

From Table 4 we notice that the curvature tensors are of two types:

(i) $a^{2} H_{1}+b^{2} H_{2}$ for $a, b \geq 0$;

(ii) $-a^{2}\left(\Omega_{\mathbb{P}_{\mathbb{C}}^{2}}+6 b H_{2}\right)$ for $a>0$ and $b \in\{0,1\}$.

A Kähler Lie group $M$ of dimension 4 is always solvable [11, Theorem 9, p. 155]. This implies that $M$ is the product of a (product of circumferences) and a Euclidean space [10, Theorem $2^{\mathrm{a}}$, p. 675], but $M$ is also simply connected, so it must be an Euclidean space, and thus contractible. If now $M$ has a projective special Kähler structure, thanks to Corollary 7.9, there is a global section $\eta: M \rightarrow \sharp_{2} S_{3,0} M$ satisfying D1* and D2*. Applying $b_{2}$ we obtain a global section $\sigma$ of $S_{3,0} M$ which better displays the symmetry.

Consider the globally defined complex coframe $\theta^{1}=u^{1}+i u^{2}, \theta^{2}=u^{3}+i u^{4}$. We write $\sigma$ in its generic form with respect to $\theta$ :

$$
\sigma=c_{1}\left(\theta^{1}\right)^{3}+c_{2}\left(\theta^{1}\right)^{2} \theta^{2}+c_{3} \theta^{1}\left(\theta^{2}\right)^{2}+c_{4}\left(\theta^{2}\right)^{3}
$$

for some functions $c_{1}, c_{2}, c_{3}, c_{4} \in \mathcal{C}^{\infty}(M, \mathbb{C})$. By raising the second index, we obtain $\eta=\#_{2} \sigma$ which is

$$
\begin{aligned}
\eta= & 2 c_{1} \theta^{1} \otimes \overline{\theta_{1}} \otimes \theta^{1}+\frac{2 c_{2}}{3}\left(\theta^{1} \otimes \overline{\theta_{1}} \otimes \theta^{2}+\theta^{1} \otimes \overline{\theta_{2}} \otimes \theta^{1}+\theta^{2} \otimes \overline{\theta_{1}} \otimes \theta^{1}\right) \\
& +\frac{2 c_{3}}{3}\left(\theta^{1} \otimes \overline{\theta_{2}} \otimes \theta^{2}+\theta^{2} \otimes \overline{\theta_{1}} \otimes \theta^{2}+\theta^{2} \otimes \overline{\theta_{2}} \otimes \theta^{1}\right)+2 c_{4} \theta^{2} \otimes \overline{\theta_{2}} \otimes \theta^{2} .
\end{aligned}
$$


Table 4 Curvature tensors

\begin{tabular}{|c|c|c|c|}
\hline Case & $\mathfrak{g}$ & Str. constants & $\Omega^{L C}$ \\
\hline I & $\mathfrak{r r}_{3,0}$ & $\begin{array}{l}{\left[u_{1}, u_{2}\right]=a u_{2}} \\
a>0\end{array}$ & $a^{2} H_{1}$ \\
\hline II & $\mathfrak{r r}_{3,0}^{\prime}$ & $\begin{array}{l}{\left[u_{1}, u_{3}\right]=-u_{4}} \\
{\left[u_{1}, u_{4}\right]=u_{3}}\end{array}$ & 0 \\
\hline III & $\mathfrak{r}_{2} \mathfrak{r}_{2}$ & $\begin{array}{l}{\left[u_{1}, u_{2}\right]=a u_{2}} \\
{\left[u_{3}, u_{4}\right]=b u_{4}} \\
a, b>0\end{array}$ & $a^{2} H_{1}+b^{2} H_{2}$ \\
\hline IV & $\mathfrak{r}_{4,0, \delta}^{\prime}$ & $\begin{array}{l}{\left[u_{1}, u_{2}\right]=a u_{2}} \\
{\left[u_{1}, u_{3}\right]=-\delta a u_{4}} \\
{\left[u_{1}, u_{4}\right]=\delta a u_{3}} \\
a, \delta>0\end{array}$ & $a^{2} H_{1}$ \\
\hline V & $\mathfrak{r}_{4,0, \delta}^{\prime}$ & $\begin{array}{l}{\left[u_{1}, u_{2}\right]=a u_{2}} \\
{\left[u_{1}, u_{3}\right]=\delta a u_{4}} \\
{\left[u_{1}, u_{4}\right]=-\delta a u_{3}} \\
a, \delta>0\end{array}$ & $a^{2} H_{1}$ \\
\hline VI & $\mathfrak{d}_{4,2}$ & $\begin{array}{l}{\left[u_{1}, u_{2}\right]=-2 a u_{1}} \\
{\left[u_{1}, u_{3}\right]=2 a u_{4}} \\
{\left[u_{2}, u_{3}\right]=-a u_{3}} \\
{\left[u_{2}, u_{4}\right]=a u_{4}} \\
a>0\end{array}$ & $-a^{2} \Omega_{\mathbb{P}_{\mathrm{C}}^{2}}-6 a^{2} H_{2}$ \\
\hline VII & $\mathfrak{d}_{4,1 / 2}$ & $\begin{array}{l}{\left[u_{1}, u_{2}\right]=2 a u_{4}} \\
{\left[u_{1}, u_{3}\right]=-a u_{1}} \\
{\left[u_{2}, u_{3}\right]=-a u_{2}} \\
{\left[u_{3}, u_{4}\right]=2 a u_{4}} \\
a>0\end{array}$ & $-a^{2} \Omega_{\mathbb{P}_{C}^{2}}$ \\
\hline VIII & $\mathfrak{b}_{4, \delta}^{\prime}$ & $\begin{array}{l}{\left[u_{1}, u_{2}\right]=2 a \sqrt{\delta} u_{4}} \\
{\left[u_{1}, u_{3}\right]=-a \sqrt{\delta} u_{1}+\frac{2 a}{\sqrt{\delta}} u_{2}} \\
{\left[u_{2}, u_{3}\right]=-\frac{2 a}{\sqrt{\delta}} u_{1}-a \sqrt{\delta} u_{2}} \\
{\left[u_{3}, u_{4}\right]=2 a \sqrt{\delta} u_{4}} \\
a, \delta>0\end{array}$ & $-\delta a^{2} \Omega_{\mathbb{P}_{\mathrm{C}}^{2}}$ \\
\hline IX & $\mathfrak{b}_{4, \delta}^{\prime}$ & $\begin{array}{l}{\left[u_{1}, u_{2}\right]=-2 a \sqrt{\delta} u_{3}} \\
{\left[u_{1}, u_{4}\right]=-a \sqrt{\delta} u_{1}-\frac{2 a}{\sqrt{\delta}} u_{2}} \\
{\left[u_{2}, u_{4}\right]=+\frac{2 a}{\sqrt{\delta}} u_{1}-a \sqrt{\delta} u_{2}} \\
{\left[u_{3}, u_{4}\right]=-2 a \sqrt{\delta} u_{3}} \\
a, \delta>0\end{array}$ & $-\delta a^{2} \Omega_{\mathbb{P}_{\mathrm{C}}^{2}}$ \\
\hline
\end{tabular}

With respect to this generic section, we can compute $[\eta \wedge \bar{\eta}]$ explicitly:

$$
\begin{aligned}
{[\eta \wedge \bar{\eta}]=} & \frac{4}{9} \Re\left(\overline{\theta^{1}} \wedge \theta^{1} \otimes\left(\begin{array}{cc}
9\left|c_{1}\right|^{2}+\left|c_{2}\right|^{2} & 3 \overline{c_{1}} c_{2}+\overline{c_{2}} c_{3} \\
3 \overline{c_{2}} c_{1}+\overline{c_{3}} c_{2} & \left|c_{2}\right|^{2}+\left|c_{3}\right|^{2}
\end{array}\right)\right. \\
& +\overline{\theta^{1}} \wedge \theta^{2} \otimes\left(\begin{array}{ll}
3 \overline{c_{1}} c_{2}+\overline{c_{2}} c_{3} & \overline{c_{1}} c_{3}+\overline{c_{2}} c_{4} \\
\left|c_{2}\right|^{2}+\left|c_{3}\right|^{2} & \overline{c_{2}} c_{3}+3 \overline{c_{3}} c_{4}
\end{array}\right) \\
& +\overline{\theta^{2}} \wedge \theta^{1} \otimes\left(\begin{array}{cc}
3 \overline{c_{2}} c_{1}+\overline{c_{3}} c_{2} & \left|c_{2}\right|^{2}+\left|c_{3}\right|^{2} \\
\overline{c_{3}} c_{1}+\overline{c_{4}} c_{2} & \overline{c_{3}} c_{2}+3 \overline{c_{4}} c_{3}
\end{array}\right) \\
& \left.+\overline{\theta^{2}} \wedge \theta^{2} \otimes\left(\begin{array}{ll}
\left|c_{2}\right|^{2}+\left|c_{3}\right|^{2} & \overline{c_{2}} c_{3}+\overline{c_{3}} c_{4} \\
\overline{c_{3}} c_{2}+3 \overline{c_{4}} c_{3} & \left|c_{3}\right|^{2}+9\left|c_{4}\right|^{2}
\end{array}\right)\right) .
\end{aligned}
$$

Notice that if we define $v_{1}, v_{2}, v_{3} \in \mathcal{C}^{\infty}\left(M, \mathbb{C}^{2}\right)$ such that 


$$
v_{1}:=\left(\begin{array}{c}
2 c_{1} \\
\frac{2 c_{2}}{3}
\end{array}\right)=\left(\begin{array}{l}
x \\
y
\end{array}\right), \quad v_{2}:=\left(\begin{array}{c}
\frac{2 c_{2}}{3} \\
\frac{2 c_{3}}{3}
\end{array}\right)=\left(\begin{array}{l}
y \\
z
\end{array}\right), \quad v_{3}:=\left(\begin{array}{c}
\frac{2 c_{3}}{3} \\
2 c_{4}
\end{array}\right)=\left(\begin{array}{c}
z \\
w
\end{array}\right),
$$

then we have

$$
\begin{aligned}
& {[\eta \wedge \bar{\eta}]=\Re\left(\overline{\theta^{1}} \wedge \theta^{1} \otimes\left(\frac{\left\|v_{1}\right\|^{2}}{\left\langle v_{1}, v_{2}\right\rangle} \begin{array}{cc}
\left\langle v_{1}, v_{2}\right\rangle & \left\|v_{2}\right\|^{2}
\end{array}\right)+\overline{\theta^{1}} \wedge \theta^{2} \otimes\left(\begin{array}{cc}
\left\langle v_{1}, v_{2}\right\rangle & \left\langle v_{1}, v_{3}\right\rangle \\
\left\|v_{2}\right\|^{2} & \left\langle v_{2}, v_{3}\right\rangle
\end{array}\right)\right.} \\
& \left.\left.+\overline{\theta^{2}} \wedge \theta^{1} \otimes\left(\overline{\frac{\left\langle v_{1}, v_{2}\right\rangle}{\left\langle v_{2}, v_{3}\right\rangle}} \frac{\left\|v_{2}\right\|^{2}}{\left\langle v_{2}, v_{3}\right\rangle}\right)+\overline{\theta^{2}} \wedge \theta^{2} \otimes\left(\frac{\left\|v_{2}\right\|^{2}}{\left\langle v_{2}, v_{3}\right\rangle} \| v_{2}, v_{3}\right\rangle\right)\right) \text {. }
\end{aligned}
$$

In other words, the coefficients of $[\eta \wedge \bar{\eta}]$ are the pairwise Hermitian products of $v_{1}, v_{2}, v_{3}$.

Returning to the classification, if we write $H_{1}, H_{2}, \Omega_{\mathbb{P}_{C}^{2}}$ with respect to the complex coframe, we notice that the positions corresponding to the mixed Hermitian products are always zero.

$$
\begin{aligned}
H_{1}= & \Re\left(\overline{\theta^{1}} \wedge \theta^{1} \otimes\left(\begin{array}{cc}
\frac{1}{2} & 0 \\
0 & 0
\end{array}\right)\right), \quad H_{2}=\mathfrak{R}\left(\overline{\theta^{2}} \wedge \theta^{2} \otimes\left(\begin{array}{ll}
0 & 0 \\
0 & \frac{1}{2}
\end{array}\right)\right), \\
\Omega_{\mathbb{P}_{\complement}^{2}}= & \Re\left(\overline{\theta^{1}} \wedge \theta^{1} \otimes\left(\begin{array}{cc}
-2 & 0 \\
0 & -1
\end{array}\right)+\overline{\theta^{1}} \wedge \theta^{2} \otimes\left(\begin{array}{cc}
0 & 0 \\
-1 & 0
\end{array}\right)\right. \\
& \left.+\overline{\theta^{2}} \wedge \theta^{1} \otimes\left(\begin{array}{cc}
0 & -1 \\
0 & 0
\end{array}\right)+\overline{\theta^{2}} \wedge \theta^{2} \otimes\left(\begin{array}{cc}
-1 & 0 \\
0 & -2
\end{array}\right)\right) .
\end{aligned}
$$

As a consequence, for all cases, if $\mathbf{D} 1$ holds, then $v_{1}, v_{2}, v_{3}$ must be orthogonal.

Now we will treat each case of possible curvature tensor separately.

(i) Let $a, b \geq 0$ and $\Omega^{L C}=a^{2} H_{1}+b^{2} H_{2}$, then

$$
\Omega^{L C}=\Re\left(\overline{\theta^{1}} \wedge \theta^{1} \otimes\left(\begin{array}{cc}
\frac{a^{2}}{2} & 0 \\
0 & 0
\end{array}\right)+\overline{\theta^{2}} \wedge \theta^{2} \otimes\left(\begin{array}{ll}
0 & 0 \\
0 & \frac{b^{2}}{2}
\end{array}\right)\right) .
$$

So, by $\mathbf{D 1},[\eta \wedge \bar{\eta}]=-\Omega^{L C}-\Omega_{\mathbb{P}_{\mathbb{C}}^{2}}$, which implies

$$
\left\|v_{1}\right\|^{2}=2-\frac{a^{2}}{2}, \quad\left\|v_{2}\right\|^{2}=1, \quad\left\|v_{3}\right\|^{2}=2-\frac{b^{2}}{2} .
$$

These equalities translate to a linear system in the squared norms of $x, y, z, w$ introduced in (16), namely

$$
\left\{\begin{array}{l}
|x|^{2}+|y|^{2}=2-\frac{a^{2}}{2} \\
|y|^{2}+|z|^{2}=1 \\
|z|^{2}+|w|^{2}=2-\frac{b^{2}}{2}
\end{array} .\right.
$$

Its solutions are 


$$
\left\{\begin{array}{l}
|x|^{2}=1-\frac{a^{2}}{2}+s \\
|y|^{2}=1-s \\
|z|^{2}=s \\
|w|^{2}=2-\frac{b^{2}}{2}-s
\end{array} \quad \text { for } s \in[0,1] .\right.
$$

Imposing the orthogonality conditions $\left\langle v_{1}, v_{2}\right\rangle=\left\langle v_{2}, v_{3}\right\rangle=\left\langle v_{3}, v_{4}\right\rangle=0$, we get:

$$
\left\{\begin{array}{l}
\bar{x} y+\bar{y} z=0 \\
\bar{y} z+\bar{z} w=0 \\
\bar{x} z+\bar{y} w=0
\end{array} .\right.
$$

Notice that because of (17), $y$ and $z$ cannot vanish simultaneously, so we have (at each point) three different cases:

- Suppose at first that $z=0$, then $s=0$ and $\|y\|=1$, so $y \neq 0$ and (18) becomes

$$
\left\{\begin{array}{l}
\bar{x} y=0 \\
0=0 \\
\bar{y} w=0
\end{array} .\right.
$$

Implying $x=w=0$, so the solutions are $(x, y, z, w)=(0, y, 0,0)$ for $y \in \mathcal{C}^{\infty}(U, \mathrm{U}(1))$. Now $M$ is simply connected, so $y=e^{i \alpha}$ for some $\alpha \in \mathcal{C}^{\infty}(M)$, as $y$ lifts to the universal cover exp $: i \mathbb{R} \rightarrow \mathrm{U}(1)$. Thus, we have $\left(c_{1}, c_{2}, c_{3}, c_{4}\right)=\left(0, \frac{3}{2} e^{i \alpha}, 0,0\right)$ for some $\alpha \in \mathcal{C}^{\infty}(M)$. Finally, (17) gives

$$
\left\{\begin{array}{l}
1-\frac{a^{2}}{2}=0 \\
2-\frac{b^{2}}{2}=0
\end{array}\right.
$$

, and thus, $a=\sqrt{2}$ and $b=2$.

- Suppose now that $z \neq 0$ and $y=0$, then (18) becomes

$$
\left\{\begin{array}{l}
0=0 \\
\bar{z} w=0 \\
\bar{x} z=0
\end{array}\right.
$$

and then $w=x=0$ so, similarly to the previous case, the solutions are $\left(c_{1}, c_{2}, c_{3}, c_{4}\right)=\left(0,0, e^{i \alpha}, 0\right)$ for $\alpha \in \mathcal{C}^{\infty}(M)$ and this time, (17) implies $a=2$ and $b=\sqrt{2}$.

- The remaining case has $z \neq 0$ and $y \neq 0$. In order to solve it, let us call $t:=\bar{y} z \neq 0$, then (17) and (18) give 


$$
\begin{aligned}
z & =\frac{t y}{|y|^{2}}=\frac{t y}{1-s}, \\
x & =-\frac{\overline{t y}}{|y|^{2}}=-\frac{\overline{t y}}{1-s}, \\
w & =-\frac{t z}{|z|^{2}}=-\frac{t^{2} y}{s(1-s)}, \\
0=\bar{x} z+\bar{y} w & =\left(-\frac{t \bar{y}}{1-s}\right)\left(\frac{t y}{1-s}\right)+\bar{y}\left(-\frac{t^{2} y}{s(1-s)}\right) \\
& =-t^{2}\left(\frac{1}{1-s}+\frac{1}{s}\right)=-\frac{t^{2}}{s(1-s)},
\end{aligned}
$$

in contradiction with $t \neq 0$.

In conclusion, for this class of curvature tensors, the only solutions are for

$$
\begin{array}{ll}
a=\sqrt{2}, \quad b=2, & \sigma=\frac{3}{2} e^{i \alpha}\left(\theta^{1}\right)^{2} \theta^{2}, \quad \text { for } \alpha \in \mathcal{C}^{\infty}(M), \text { or } \\
a=2, & b=\sqrt{2}, \quad \sigma=\frac{3}{2} e^{i \alpha} \theta^{1}\left(\theta^{2}\right)^{2}, \quad \text { for } \alpha \in \mathcal{C}^{\infty}(M) .
\end{array}
$$

We deduce that in Table 4 there are no solutions for the cases I, II, IV, V, and the only solutions in case III are the ones mentioned before. Moreover, these solutions are isomorphic to one another and the isomorphism is obtained by swapping $u_{1}$ with $u_{3}$ and $u_{2}$ with $u_{4}$. The simply connected Lie group corresponding to this case is $\mathcal{H}_{\sqrt{2}} \times \mathcal{H}_{2}$. Notice that the unique abelian Kähler 4-dimensional Lie algebra is flat, so its curvature is also of type (i), with $a=b=0$; thus, it cannot be endowed with a projective special Kähler structure.

(ii) Let now $a>0, b \in\{0,1\}$ and $\Omega^{L C}=-a^{2}\left(\Omega_{\mathbb{P}_{\complement}^{2}}+6 b H_{2}\right)$, then

$$
\begin{aligned}
& {[\eta \wedge \bar{\eta}]=-\Omega^{L C}-\Omega_{\mathbb{P}_{\mathbb{C}}^{n}}=\left(a^{2}-1\right) \Omega_{\mathbb{P}_{\mathbb{C}}^{n}}+6 a^{2} b H_{2}} \\
& =\Re\left(\overline{\theta^{1}} \wedge \theta^{1} \otimes\left(\begin{array}{cc}
2\left(1-a^{2}\right) & 0 \\
0 & 1-a^{2}
\end{array}\right)+\overline{\theta^{1}} \wedge \theta^{2} \otimes\left(\begin{array}{cc}
0 & 0 \\
1-a^{2} & 0
\end{array}\right)\right. \\
& \left.+\overline{\theta^{2}} \wedge \theta^{1} \otimes\left(\begin{array}{cc}
0 & 1-a^{2} \\
0 & 0
\end{array}\right)+\overline{\theta^{2}} \wedge \theta^{2} \otimes\left(\begin{array}{cc}
1-a^{2} & 0 \\
0 & 2-2 a^{2}+3 a^{2} b
\end{array}\right)\right) \text {. }
\end{aligned}
$$

Therefore, we obtain equations

$$
\left\|v_{1}\right\|^{2}=2-2 a^{2}, \quad\left\|v_{2}\right\|^{2}=1-a^{2}, \quad\left\|v_{3}\right\|^{2}=2-2 a^{2}+3 a^{2} b .
$$

Giving the conditions

$$
\left\{\begin{array}{l}
|x|^{2}+|y|^{2}=2-2 a^{2} \\
|y|^{2}+|z|^{2}=1-a^{2} \\
|z|^{2}+|w|^{2}=2-2 a^{2}+3 a^{2} b
\end{array}\right.
$$

with solutions 


$$
\left\{\begin{array}{l}
|x|^{2}=1-a^{2}+s \\
|y|^{2}=1-a^{2}-s \\
|z|^{2}=s \\
|w|^{2}=2-2 a^{2}+3 a^{2} b-s
\end{array} \quad \text { for } s \in\left[0,1-a^{2}\right] .\right.
$$

We now impose the vanishing of $\left\langle v_{1}, v_{2}\right\rangle,\left\langle v_{2}, v_{3}\right\rangle,\left\langle v_{3}, v_{4}\right\rangle$, that is (18). We have four different cases:

- Suppose at first that $y=z=0$, then $s=0$ and $a=1$, so (18) is always satisfied, while (19) becomes

$$
\left\{\begin{array}{l}
|x|^{2}=0 \\
|y|^{2}=0 \\
|z|^{2}=0 \\
|w|^{2}=3 b
\end{array}\right.
$$

It has solutions $(x, y, z, w)=\left(0,0,0, \sqrt{3 b} e^{i \alpha}\right)$ for $\alpha \in \mathcal{C}^{\infty}(M)$, and thus, $\left(c_{1}, c_{2}, c_{3}, c_{4}\right)=\left(0,0,0, \frac{\sqrt{3 b}}{2} e^{i \alpha}\right)$. In conclusion, $a=1$ and $\sigma=\frac{\sqrt{3 b}}{2} e^{i \alpha}\left(\theta_{2}\right)^{3}$.

- Suppose now that $z=0$ but $y \neq 0$, then $s=0$ and $a^{2}-1 \neq 0$. The system (18) implies $x=w=0$, but then by (19), $0=|x|^{2}=1-a^{2} \neq 0$, so in this case there are no solutions.

- Analogously, if $z \neq 0$ but $y=0$, then $s=1-a^{2}$ and (18) gives $w=x=0$, so from (19) we get $0=|x|^{2}=2-2 a^{2}=2|z|^{2} \neq 0$ leaving no solutions.

- The remaining case has $z \neq 0$ and $y \neq 0$. In order to solve it, let us call $t:=\bar{y} z \neq 0$, then (19) and (18) give

$$
\begin{aligned}
z & =\frac{t y}{|y|^{2}}=\frac{t y}{1-a^{2}-s}, \\
x & =-\frac{\bar{t} y}{|y|^{2}}=-\frac{\bar{t} y}{1-a^{2}-s}, \\
w & =-\frac{t z}{|z|^{2}}=-\frac{t^{2} y}{s\left(1-a^{2}-s\right)}, \\
0=\bar{x} z+\bar{y} w & =\left(\frac{-t \bar{y}}{1-a^{2}-s}\right)\left(\frac{t y}{1-a^{2}-s}\right)+\bar{y}\left(\frac{-t^{2} y}{s\left(1-a^{2}-s\right)}\right) \\
& =-t^{2}\left(\frac{1}{1-a^{2}-s}+\frac{1}{s}\right)=-\frac{t^{2}\left(1-a^{2}\right)}{s\left(1-a^{2}-s\right)} .
\end{aligned}
$$

The latter implies $a=1$, and from (19), we deduce a contradiction: $0<|y|^{2}=-s<0$. for

In conclusion, the only solutions for this type of curvature tensors are obtained

$$
\begin{aligned}
& a=1, \quad b=0, \quad \sigma=0, \quad \text { or } \\
& a=1, \quad b=1, \quad \sigma=\frac{\sqrt{3}}{2} e^{i \alpha}\left(\theta^{2}\right)^{3}, \quad \text { for } \alpha \in \mathcal{C}^{\infty}(M) .
\end{aligned}
$$

In Table 4, these results correspond to: VI for $a=1$ and $\sigma=\frac{\sqrt{3}}{2} e^{i \alpha}\left(\theta^{2}\right)^{3}$ for $\alpha \in \mathcal{C}^{\infty}(M)$; VII for $a=1$ and $\sigma=0$; VIII and IX for $a=\frac{1}{\sqrt{\delta}}, \delta>0$ and $\sigma=0$. 
Table 5 Cases satisfying the curvature condition

\begin{tabular}{|c|c|c|c|c|}
\hline Case & Structure constants & Levi-Civita connection & & PSK \\
\hline III & $\begin{aligned} d u^{2} & =-\sqrt{2} u^{1,2} \\
d u^{4} & =-2 u^{3,4}\end{aligned}$ & $\left(-\sqrt{2} u^{2}{ }^{\sqrt{2} u^{2}}\right.$ & $4^{2} u^{4}$ & $\checkmark$ \\
\hline VI & $\begin{aligned} d u^{1} & =2 u^{1,2} \\
d u^{3} & =u^{2,3} \\
d u^{4} & =-2 u^{1,3}-u^{2,4}\end{aligned}$ & $\left(\begin{array}{cc|cc}0 & -2 u^{1} & u^{4} & u \\
2 u^{1} & 0 & -u^{3} & u \\
-u^{4} & u^{3} & 0 & - \\
-u^{3} & -u^{4} & u^{1} & \end{array}\right.$ & $\left.\begin{array}{l}c^{3} \\
u^{4} \\
u^{1} \\
0\end{array}\right)$ & \\
\hline VII & $\begin{aligned} d u^{1} & =u^{1,3} \\
d u^{2} & =u^{2,3} \\
d u^{4} & =-2 u^{1,2}-2 u^{3,4}\end{aligned}$ & $\left(\begin{array}{cc|cc}0 & u^{4} & -u^{1} & u \\
-u^{4} & 0 & -u^{2} & - \\
u^{1} & u^{3} & 0 & 2 v \\
-u^{2} & -u^{4} & -2 u^{4} & c\end{array}\right.$ & $\left.\begin{array}{l}u^{2} \\
u^{1} \\
u^{4} \\
0\end{array}\right)$ & $\checkmark$ \\
\hline VIII & $\begin{array}{l}d u^{1}=u^{1,3}+\frac{2}{\delta} u^{2,3} \\
d u^{2}=-\frac{2}{\delta} u^{1,3}+u^{2,3} \\
d u^{4}=-2 u^{1,2}-2 u^{3,4} \\
\delta>0\end{array}$ & $\left(\begin{array}{cc}0 & \frac{2}{\delta} u^{3}+u^{4} \\
-\frac{2}{\delta} u^{3}-u^{4} & 0 \\
u^{1} & u^{2} \\
-u^{2} & u^{1}\end{array} \mid\right.$ & $\left.\begin{array}{cc}-u^{1} & u^{2} \\
-u^{2} & -u^{1} \\
0 & 2 u^{4} \\
-2 u^{4} & 0\end{array}\right)$ & $\checkmark$ \\
\hline IX & $\begin{array}{l}d u^{1}=u^{1,4}-\frac{2}{\delta} u^{2,4} \\
d u^{2}=\frac{2}{\delta} u^{1,4}+u^{2,4} \\
d u^{3}=2 u^{1,2}+2 u^{3,4} \\
\delta>0\end{array}$ & $\left(\begin{array}{cc}0 & -\frac{2}{\delta} u^{4}-u^{3} \\
\frac{2}{\delta} u^{4}+u^{3} & 0 \\
u^{2} & -u^{1} \\
u^{1} & u^{2}\end{array}\right.$ & $\begin{array}{cc}-u^{2} & -u^{1} \\
u^{1} & -u^{2} \\
0 & -2 u^{3} \\
2 u^{3} & 0\end{array}$ & $\checkmark$ \\
\hline
\end{tabular}

Table 5 summarises (up to isomorphisms) the cases satisfying the curvature condition, showing the non-vanishing differentials of the coframe and the Levi-Civita connection.

Now we must check whether condition D2 holds for the cases left. Notice that for cases III, VII, VIII, IX, the Kähler form is exact with invariant potentials, respectively $-\frac{1}{\sqrt{2}} u^{2}-\frac{1}{2} u^{4},-\frac{1}{2} u^{4},-\frac{1}{2} u^{4}, \frac{1}{2} u^{3}$. We can immediately say that cases VII, VIII, IX are all projective special Kähler because $\sigma=0$, so the differential condition is trivially satisfied.

Concerning case III, we can compute $d^{L C} \sigma$ by understanding how the Levi-Civita connection behaves on the unitary complex coframe $\theta$.

$$
\begin{aligned}
\nabla^{L C} \theta^{1} & =\nabla^{L C} u^{1}+i \nabla^{L C} u^{2}=-\left(\omega^{L C}\right)_{k}^{1} \otimes u^{k}-i\left(\omega^{L C}\right)_{k}^{2} \otimes u^{k} \\
& =-\sqrt{2} u^{2} \otimes u^{2}+i \sqrt{2} u^{2} \otimes u^{1}=\sqrt{2} i u^{2} \otimes \theta^{1} ; \\
\nabla^{L C} \theta^{2} & =\nabla^{L C} u^{3}+i \nabla^{L C} u^{4}=-\left(\omega^{L C}\right)_{k}^{3} \otimes u^{k}-i\left(\omega^{L C}\right)_{k}^{4} \otimes u^{k} \\
& =-2 u^{4} \otimes u^{4}+i 2 u^{4} \otimes u^{3}=2 i u^{4} \otimes \theta^{2} .
\end{aligned}
$$

Now we can compute 


$$
\begin{aligned}
\nabla^{L C} \sigma & =\nabla^{L C}\left(\frac{3}{2} e^{i \alpha}\left(\theta^{1}\right)^{2} \theta^{2}\right) \\
& =\frac{3}{2} i d \alpha \otimes e^{i \alpha}\left(\theta^{1}\right)^{2} \theta^{2}+3 \sqrt{2} i u^{2} e^{i \alpha}\left(\theta^{1}\right)^{2} \theta^{2}+\frac{3}{2} 2 i u^{4} \otimes e^{i \alpha}\left(\theta^{1}\right)^{2} \theta^{2} \\
& =-4 i\left(-\frac{1}{4} d \alpha-\frac{1}{\sqrt{2}} u^{2}-\frac{1}{2} u^{4}\right) \otimes \sigma
\end{aligned}
$$

If we define $\lambda:=-\frac{1}{4} d \alpha-\frac{1}{\sqrt{2}} u^{2}-\frac{1}{2} u^{4}$, we have that $d \lambda=\omega$ and $d^{L C} \sigma=-4 i \lambda \wedge \sigma$. Thanks to Corollary 7.9, we have proven that also case III has a projective special Kähler structure for every choice of $\alpha \in \mathcal{C}^{\infty}(M)$.

Suppose that VI is projective special Kähler, than by Theorem 7.6, locally we must have the differential condition D2. Consider the unitary global complex coframe $\theta$.

$$
\begin{aligned}
\nabla^{L C} \theta^{2} & =\nabla^{L C} u^{3}+i \nabla^{L C} u^{4} \\
& =u^{4} \otimes u^{1}-u^{3} \otimes u^{2}+u^{1} \otimes u^{4}+i\left(u^{3} \otimes u^{1}+u^{4} \otimes u^{2}-u^{1} \otimes u^{3}\right) \\
& =u^{4} \otimes \theta^{1}+i u^{3} \otimes \theta^{1}-i u^{1} \otimes \theta^{2}=i \overline{\theta^{2}} \otimes \theta^{1}-i u^{1} \otimes \theta^{2}
\end{aligned}
$$

Thus,

$$
\begin{aligned}
\nabla^{L C} \sigma & =\nabla^{L C}\left(\frac{\sqrt{3}}{2} e^{i \alpha}\left(\theta^{2}\right)^{3}\right) \\
& =i d \alpha \otimes \frac{\sqrt{3}}{2} e^{i \alpha}\left(\theta^{2}\right)^{3}+3 \frac{\sqrt{3}}{2} e^{i \alpha}\left(\nabla^{L C} \theta^{2}\right)\left(\theta^{2}\right)^{2} \\
& =i d \alpha \otimes \sigma+3 \frac{\sqrt{3}}{2} e^{i \alpha}\left(i \overline{\theta^{2}} \otimes \theta^{1}-i u^{1} \otimes \theta^{2}\right)\left(\theta^{2}\right)^{2} \\
& =i\left(d \alpha-3 u^{1}\right) \otimes \sigma+3 i \overline{\theta^{2}} \otimes \frac{\sqrt{3}}{2} e^{i \alpha} \theta^{1}\left(\theta^{2}\right)^{2} \\
d^{L C} \sigma & =i\left(d \alpha-3 u^{1}\right) \wedge \sigma+3 i \overline{\theta^{2}} \wedge \frac{\sqrt{3}}{2} e^{i \alpha} \theta^{1}\left(\theta^{2}\right)^{2} .
\end{aligned}
$$

Notice that this is never of the form required by condition D2 for any available choice of $\sigma$, since evaluating the last component at $\theta_{1}$, we obtain $i \frac{\sqrt{3}}{2} \overline{\theta^{2}} \wedge \theta^{2} \otimes \theta^{2}$, whereas the same operation on a form of type $i \tau \wedge \sigma$ would evaluate to zero. We deduce that VI does not admit a projective special Kähler structure.

We are now left with cases III, VII, VIII, IX. At the level of Lie groups, case III corresponds to the connected simply connected Lie group $\mathcal{H}_{\sqrt{2}} \times \mathcal{H}_{2}$ with $\sigma=\frac{3}{2}\left(\theta^{1}\right)^{2} \theta^{2}$ up to isomorphism. The other deviances are in fact obtained by taking $e^{i \alpha} \sigma$, and thus, we are in the situation noted in Remark 8.4. The Lie groups corresponding to the cases VII, VIII and IX are in particular homogeneous, and they all have zero deviance, so by Proposition 9.5 we deduce that they are all isomorphic to $\mathcal{H}_{\mathbb{C}}^{2}$ as projective special Kähler manifolds.

Remark 10.3 It is striking that in case III, which is obtained via the r-map from the polynomial $x^{2} y$, the deviance is a global tensor which is a multiple of this polynomial with respect to a Kähler holomorphic coframe. 
It turns out that all 4-dimensional projective special Kähler Lie groups are simply connected, so this theorem already presents all possible cases.

Proposition 10.4 Let $(\pi: \widetilde{M} \rightarrow M, \nabla)$ be a projective special Kähler manifold, then the universal cover $p: U \rightarrow M$ admits a projective special Kähler structure. In particular, if $\gamma: S \rightarrow \sharp_{2} S_{3,0} M$ is the intrinsic deviance for $M$, then $p^{*} S \rightarrow U$ is an $S^{1}$-bundle and if we call $p^{\prime}$ the canonical map $p^{*} S \rightarrow S$, then $U$ has deviance $p^{*}$ o $\gamma \circ p^{\prime}: p^{*} S \rightarrow \sharp_{2} S_{3,0} U$ on $U$.

If $M$ is a projective special Kähler Lie group, then so is $U$.

Proof Since $p: U \rightarrow M$ is a cover, we can lift the whole Kähler structure of $M$ to $U$ by pullback $\left(U, p^{*} g, p^{*} I, p^{*} \omega\right)$ (the pullback of $I$ makes sense, since $p$ is a local diffeomorphism). We will now use Theorem 7.6. The $S^{1}$-bundle $S$ lifts to an $S^{1}$-bundle $\pi_{p^{*} S}: p^{*} S \rightarrow U$, where the right action can be defined locally, since $p$ is a local diffeomorphism. The principal connection $\varphi$ on $S$ lifts to $\varphi^{\prime}=p^{\prime *} \varphi$ and its curvature is, as expected, $d \varphi^{\prime}=p^{*} d \varphi=-2 p^{\prime} \pi_{S}^{*} \omega=-2 \pi_{p^{*} S}^{*} p^{*} \omega$. Let $\gamma^{\prime}=p^{*} \circ \gamma \circ p^{\prime}: p^{*} S \rightarrow \sharp_{2} S_{3,0} U$, then $\gamma^{\prime}(u a)=a^{2} \gamma^{\prime}(u)$ holds, as the action is defined on the fibres, which are preserved by the pullback. The remaining properties also follow from the fact $p$ is a local diffeomorphism.

Finally, if $M$ is a Lie group with left invariant Kähler structure, then $U$ is a Lie group and its Kähler structure is also left invariant.

Given a universal cover $p: U \rightarrow M$ of a projective special Kähler Lie group, $\operatorname{ker}(p)$ is a discrete subgroup and when $M$ is connected, $\operatorname{ker}(p)$ is in the centre $Z(U)$ of $U$.

From this observation, we obtain the following corollary

Corollary 10.5 A connected 4-dimensional projective special Kähler Lie group is isomorphic to one of the following:

- $\mathcal{H}_{\sqrt{2}} \times \mathcal{H}_{2}$ with deviance $\sharp_{2}\left(\frac{3}{2}\left(\theta^{1}\right)^{2} \theta^{2}\right)$ in the standard complex unitary coframe $\theta$;

- complex hyperbolic 2 -space with zero deviance.

Proof The proof follows from Theorem 10.2 with Proposition 10.4, as a connected group $M$ with universal cover $p: U \rightarrow M$ is isomorphic to $U / \operatorname{ker}(p)$ and, if $M$ is a projective special Kähler Lie group, so is $U$ by Proposition 10.4. Since $U$ is also simply connected, Theorem 10.2 provides all the possibilities up to isomorphisms preserving the Lie structure. The statement follows from the fact that these possibilities for $U$ have trivial centre.

Acknowledgements This paper is part of the author's PhD thesis [27] written under the supervision of Diego Conti. Part of the study leading to this work was carried out during a visiting period at QGM, Aarhus; the author wishes to thank Andrew Swann for many useful discussions during that visit.

Funding Open access funding provided by Università degli Studi di Milano - Bicocca within the CRUICARE Agreement.

Open Access This article is licensed under a Creative Commons Attribution 4.0 International License, which permits use, sharing, adaptation, distribution and reproduction in any medium or format, as long as you give appropriate credit to the original author(s) and the source, provide a link to the Creative Commons licence, and indicate if changes were made. The images or other third party material in this article 
are included in the article's Creative Commons licence, unless indicated otherwise in a credit line to the material. If material is not included in the article's Creative Commons licence and your intended use is not permitted by statutory regulation or exceeds the permitted use, you will need to obtain permission directly from the copyright holder. To view a copy of this licence, visit http://creativecommons.org/licenses/by/4.0/.

\section{References}

1. Alekseevsky, D.V., Cortés, V.: Classification of stationary compact homogeneous special pseudo-Kähler manifolds of semisimple groups. Proc. London Math. Soc. (3) 81(1), 211-230 (2000)

2. Alekseevsky, D.V., Cortés, V., Devchand, C.: Special complex manifolds. J. Geom. Phys. 42(1-2), 85-105 (2002)

3. Alekseevsky, D.V., Cortés, V., Dyckmanns, M., Mohaupt, T.: Quaternionic Kähler metrics associated with special Kähler manifolds. J. Geom. Phys. 92, 271-287 (2015)

4. Alekseevsky, D.V., Cortés, V., Mohaupt, T.: Conification of Kähler and hyper-Kähler manifolds. Comm. Math. Phys. 324(2), 637-655 (2013)

5. Baues, O., Cortés, V.: Abelian simply transitive affine groups of symplectic type. Ann. Inst. Fourier (Grenoble) 52(6), 1729-1751 (2002)

6. Baues, O., Cortés, V.: Proper affine hyperspheres which fiber over projective special Kähler manifolds. Asian J. Math. 7(1), 115-132 (2003)

7. Berger, M.: Sur les groupes d'holonomie homogène des variétés à connexion affine et des variétés riemanniennes. Bull. Soc. Math. France 83, 279-330 (1955)

8. Candelas, P., de la Ossa, X.C.: Moduli space of Calabi-Yau manifolds. Nuclear Phys. B 355(2), 455-481 (1991)

9. Cecotti, S., Ferrara, S., Girardello, L.: Geometry of type II superstrings and the moduli of superconformal field theories. Internat. J. Modern Phys. A 4(10), 2475-2529 (1989)

10. Chevalley, C.: On the topological structure of solvable groups. Ann. of Math. 2(42), 668-675 (1941)

11. Chu, B.Y.: Symplectic homogeneous spaces. Trans. Amer. Math. Soc. 197, 145-159 (1974)

12. Cortés, V.: On hyper-Kähler manifolds associated to Lagrangian Kähler submanifolds of $T^{*} C^{n}$. Trans. Amer. Math. Soc. 350(8), 3193-3205 (1998)

13. Cortés, V., Dyckmanns, M., Lindemann, D.: Classification of complete projective special real surfaces. Proc. Lond. Math. Soc. (3) 109(2), 423-445 (2014)

14. Cortés, V., Dyckmanns, M., Suhr, S.: Completeness of projective special Kähler and quaternionic Kähler manifolds. In Special metrics and group actions in geometry, volume 23 of Springer INdAM Ser., pages 81-106. Springer, Cham (2017)

15. Cortés, V., Han, X., Mohaupt, T.: Completeness in supergravity constructions. Comm. Math. Phys. 311(1), 191-213 (2012)

16. Cortés, V., Dyckmanns, M., Jüngling, M., Lindemann, D.: A class of cubic hypersurfaces and quaternionic Kähler manifolds of co-homogeneity one. arXiv:1701.7882, (2018)

17. Dorfmeister, J., Nakajima, K.: The fundamental conjecture for homogeneous Kähler manifolds. Acta Math. 161(1-2), 23-70 (1988)

18. Ferrara, S., Sabharwal, S.: Quaternionic manifolds for type II superstring vacua of Calabi-Yau spaces. Nuclear Phys. B 332(2), 317-332 (1990)

19. Fré, P.: Lectures on special Kähler geometry and electric-magnetic duality rotations. Nuclear Phys. B Proc. Suppl., 45BC:59-114, (1996)

20. Freed, D.S.: Special Kähler manifolds. Comm. Math. Phys. 203(1), 31-52 (1999)

21. Goldman, W.M.: Complex hyperbolic geometry. Oxford Mathematical Monographs. The Clarendon Press, Oxford University Press, New York. Oxford Science Publications (1999)

22. Helgason, S.: Differential geometry, Lie groups, and symmetric spaces, volume 34 of Graduate Studies in Mathematics. American Mathematical Society, Providence, RI (2001). Corrected reprint of the 1978 original

23. Hitchin, N.: On the hyperkähler/quaternion Kähler correspondence. Comm. Math. Phys. 324(1), 77-106 (2013)

24. Kobayashi, S., Nomizu, K.: Foundations of Differential Geometry. Intersciences Publishers, New York. Springer-Verlag, $(1963,1969)$

25. Macia, O., Swann, A.: The c-map on groups. Class. Quantum Gravity 37(1), 015015 (2020)

26. Macia, O., Swann, A.: Twist geometry of the c-map. Comm. Math. Phys. 336(3), 1329-1357 (2015)

27. Mantegazza, M.: An intrinsic approach to the c-map. PhD thesis, Joint PhD program in mathematics, Università degli studi di Pavia, Università degli studi di Milano-Bicocca, Indam, 12 (2019)

28. Ovando, G.: Complex, symplectic and Kähler structures on four dimensional Lie groups. Rev. Un. Mat. Argentina 45(2), 55-67 (2005) 2004 
29. Salamon, S.: Riemannian geometry and holonomy groups, volume 201 of Pitman Research Notes in Mathematics Series. Longman Scientific \& Technical, Harlow; copublished in the United States with John Wiley \& Sons, Inc., New York, (1989)

30. Shima, H.: Vanishing theorems for compact Hessian manifolds. Ann. Inst. Fourier (Grenoble) 36(3), 183205 (1986)

31. Shima, H.: Hessian manifolds of constant Hessian sectional curvature. J. Math. Soc. Japan 47(4), 735-753 (1995)

32. Tu, L.W.: Differential geometry, volume 275 of Graduate Texts in Mathematics. Springer, Cham, (2017). Connections, curvature, and characteristic classes

Publisher's Note Springer Nature remains neutral with regard to jurisdictional claims in published maps and institutional affiliations. 